\title{
A Novel Error Equivalence Model on the Kinematic Error of the Linear Axis of High-End Machine Tool
}

\section{Xinxin LI}

Shanghai Jiao Tong University

Zhi-Min Li ( $\nabla$ zmli@sjtu.edu.cn )

Shanghai Jiao Tong University https://orcid.org/0000-0002-9794-7158

Sun Jin

Shanghai Jiao Tong University

Jichang Zhang

Shanghai Jiao Tong University

\section{Siyi Ding}

Dong Hua University

\section{Zhihua Niu}

Shanghai Jiao Tong University

\section{Research Article}

Keywords: Linear axis, Kinematic error, Error equivalence, Small displacement torsor

Posted Date: March 23rd, 2021

DOl: https://doi.org/10.21203/rs.3.rs-328470/v1

License: (c) (1) This work is licensed under a Creative Commons Attribution 4.0 International License. Read Full License

Version of Record: A version of this preprint was published at The International Journal of Advanced Manufacturing Technology on September 24th, 2021. See the published version at https://doi.org/10.1007/s00170-021-07941-3. 


\title{
A novel error equivalence model on the kinematic error of the linear axis of high-end machine tool
}

\author{
Xinxin $\mathrm{LI}^{1}$, Zhimin $\mathrm{LI}^{2, *}$, Sun JIN ${ }^{1}$, Jichang ZHANG ${ }^{1,3}$, Siyi DING ${ }^{4}$, Zhihua NIU ${ }^{1}$ \\ ${ }^{1}$ State Key Laboratory of Mechanical System and Vibration \& Shanghai Key Laboratory of \\ Digital Manufacture for Thin-Walled Structures, Shanghai Jiao Tong University, Shanghai \\ 200240, P.R. China \\ ${ }^{2}$ State Key Laboratory of Ocean Engineering, School of Naval Architecture, Ocean and Civil \\ Engineering, Shanghai Jiao Tong University, Shanghai 200240, P.R. China \\ ${ }^{3}$ Shanghai SmartState Technology Co.,Ltd, Pudong new area 201306, Shanghai, P.R. China \\ ${ }^{4}$ Institute of Intelligent Manufacturing, School of Mechanical Engineering, Dong Hua \\ University, Shanghai (201600), China
}

\begin{abstract}
The kinematic errors of the linear axis play a key role in machining precision of high-end CNC (Computer Numerical Control) machine tool. The quantification of error relationship is still an urgent problem to be solved in the assembly process of the linear axis, especially considering the effect of the elastic deformation of rollers. A systematic error equivalence model of slider is proposed to improve the prediction accuracy for kinematic errors of the linear axis which contains the base, the linear guide rail and carriage. Firstly, the geometric errors of assembly surface of linear guide rail are represented by small displacement torsor. According to the theory of different motion of robots, the error equivalence model of a single slider is established, namely the geometric error of assembly surface of linear guide rail and the pose error of slider is equivalent to the elastic deformation of roller. Based on the principle
\end{abstract}

\footnotetext{
* Corresponding author. Email: zmli@sjtu.edu.cn (Z.-M. Li)
} 
of vector summation, the kinematic error of a single slider is mapped to the carriage and the kinematic error of the linear axis is obtained. Besides, experiments validation of kinematic error model of the linear axis is carried out. It is indicated that the proposed model is accurate and feasible. The proposed model can provide an accurate guidance for the manufacturing and operation performance of the linear axis in quantification, and a more effective reference for the engineers at the design and assembly stage.

Keywords: Linear axis; Kinematic error; Error equivalence; Small displacement torsor

\section{Introduction}

With the rapid development of precision manufacturing and unceasing enhancement demand, high-precision machining centers, namely multi-axis CNC machine tools, are widely used in all kinds of advanced manufacturing fields, such as automotive engines, aero-engines and gearboxes, valve bodies and other pivotal components. Multi-axis precision CNC machine tools are composed of the linear axis, rotation axis and other components. As an important translational component and with bearing capacity, the linear axis plays a crucial role in the machining accuracy of workpieces. Generally, the horizontal CNC machine tool with four axes consists of three linear axes ( $x, y$ and $z$ axis) and a rotation axis. The linear axis is composed of a base, two linear guide rails, four sliders and a carriage. The kinematic error of the linear axis is mainly affected by the geometric error of the base and the kinematic error of the slider, meanwhile it also directly affects the machining accuracy of workpieces. However, the geometric tolerances of the installing surface of the linear axis are often given according to the designers' experience, moreover the influence factors of roller deformation inside the slider are ignored in the actual working situations. Therefore, in the design stage and manufacturing process, an accurate kinematic error prediction model of the linear axis is extremely essential. In addition, the kinematic error and machining accuracy of the linear axis can be improved from the aspects of design, manufacture and assembly techniques.

In order to obtain the kinematic errors of the linear axis, the kinematic errors of a single slider moving along the guide rail need to be acquired first. Over the past few decades, a large 
number of studies have been carried out on the kinematic errors of a single slider. There exist three categories of mainstream research. The first one is that only the geometric errors of the linear guide rail are considered, the contact analysis between the guide rail and slider is ignored. With the measuring form errors of guideway tier, He et al. [1] proposed a systematic method of motion error estimation of a linear motion bearing table. Zha et al. [2] investigated the relationship between the profile error of guide rail and vertical motion straightness of $y$ axis slider, and provide theoretic guidance for the precision design of open hydrostatic guideways. Xue et al. [3] presented a new method to analyze the motion error of a closed hydrostatic guideway and found that the wavelength of each profile error component is the main affecting factor. Considering the geometric profile error of guideway, Fan et al. [4] introduced a systematic approach to predict the kinematic error of machine tool's guideway based on the guideway tolerance. In view of the installing base bending shape, Zhong et al. [5] established the error transfer model to obtain the accurate kinematic errors of a translational axis of machine tool. Zhang et al. [6] proposed a new approximate model to study the effect of geometric errors of guide rails and table on the motion errors of hydrostatic guideways, the result showed that the geometric error of guide rail is the main influence factor. The second one is that only the contact analysis between the guide rails and slides is performed to study the kinematic errors of linear guide rail, the geometric errors of guide rail and base are neglected. Using the finite element analysis, Chlebus et al. [7] presented a method to obtain the static properties of guideway joints with contact layers. Paweł Majda [8] introduced an analytical examination approach, aiming to study the influence of static characteristics of linear guideway on the joint kinematic errors of machine tool table. Based on Hertz contact theory, the elastic deformation of guideway and roller were considered to construct the static model to study the kinematic errors of linear guideway [9, 10]. Jeong et al. [11] established the eight-spring equivalent model to link the motion components and guideways, and adopted the finite element method to analyze the static and dynamic properties of linear guideway. Zou and Wang [12] investigate the contact stiffness variation of linear rolling guides due to the effect of friction and wear, and indirectly analyzed the precision condition of linear rolling 
guides by the mode shape. The third one is that the geometric error and elastic deformation of guide rail are both considered to study the kinematic errors of linear guide rail, however the research object and the key point are different. Different from the line contact form of the linear guide rail with rollers, the linear guide rail with rolling ball is the point contact form. Ma et al. [13] proposed a method of combining the geometric error of rolling guide and the elastic deformation of rolling ball, aiming to study the motion error rule of slider under different working situations. Khim et al. [14] established the transfer function between the geometric error of the guide rail and the restoring force, and also the balance equation in the motion process to analyze the motion error of the linear guide rail. They presented a simple and systematic estimation method of motion errors of 5-DOF aerostatic linear motion stage. In view of the difficulty in obtaining the geometric error of guide rail, Khim et al. [15] further substituted the geometric error of guide rail with the straightness error of base, and analyzed the motion error of linear guide rail. As for the aerostatic bearings, Ekinci et al. [16] proposed an equation system, aiming at investigating the relationship from the geometric errors of guideway to the motion errors of axis. Khim et al. [18] introduced a prediction model of motion accuracy of a multi-sliders motion table and constructed the transfer function between the form errors of rail and the bearing force of a bearing block.

Many researches have been carried out about the geometric errors modeling and measurement of linear guideway, also the relationship between the geometric error of guideway and the kinematic error of the linear axis. Generally, the processing position change of workpiece is mainly guaranteed by the linear axis precision, so it is very essential to obtain the kinematic error of linear axis. Furthermore, the kinematic error of the linear axis is influenced by the assembly process, including the hierarchical error transfer of base, guide rail and carriage. More importantly, the kinematic error prediction model of the linear axis of machine tool can provide an accurate guidance for precision design of new machine tools.

In order to obtain the kinematic error of linear axis, a systematic error equivalence model is proposed. Firstly, the geometric errors of assembly surface of linear guide rail are represented by small displacement torsor. Then, according to the theory of different motion of 
robots, the error equivalence model of a single slider is established. Little work focused on the error equivalence method, the contribution point of the proposed model is that the geometric error of assembly surface of linear guide rail and the pose error of slider are equivalent to the elastic deformation of roller. Afterwards, based on the principle of vector summation, the kinematic error of a single slider is mapped to the carriage and the kinematic error of the linear axis is obtained. Finally, a measuring experiment of the kinematic errors of the linear axis is performed, aiming to verify the validity of the proposed model by comparing the theoretical results and the experimental values. The rest of this paper is organized as follows: Section 2 gives a geometric errors representation of linear guide rail based on MCS (Monte Carlo Simulation) method. Section 3 proposes an error equivalence model of guide rail. The contribution point is that the geometric error of guide rail and the pose error of slider are equivalent to the elastic deformation of rollers. Subsequently, the mapping model of the kinematic errors from a single slider to the linear axis is established. Finally, the predict model of kinematic errors of linear axis is obtained. Section 4 performs a measuring experiment to verify the validity of the proposed model by comparing the theoretical results and experimental values. Section 5 discusses the influence factors on the kinematic errors of linear axis. Section 6 draws conclusions and plans the future work.

\section{Geometric error representation based on MCS method}

The linear axis of machine tool is mainly composed of base, guide rail, slider, roller, carriage and other parts, the carriage reciprocates along the direction of the guide rail. The kinematic error of linear axis depends on the geometric error and deformation of the parts. The geometric error value of base and linear guide rail is unknown in the design stage, while its tolerance value is the interval value that restricts its change. The geometric tolerance is easier to obtain, but it is difficult for the precise distribution function of the geometric error. The sampling statistical analysis method is often used to analyze its uncertain parameters under the unknown distribution function. Therefore, the interval parameters and small 
displacement torsor method are adopted to describe the uncertain geometric error. Besides, MCS method is employed for simulation error, the geometric error distribution of linear guide rail is obtained under meeting the geometric tolerance constraints.

\subsection{Small displacement torsor description}

The kinematic error of the linear axis of machine tool usually depends on the manufacturing error, bearing deformation and assembly error of the parts, but the geometric error is uncertain, so it is obviously difficult to study. Hence, some characteristic parameters are employed to describe the geometric elements, and then are transformed into the study of the uncertain geometric error.

Torsor theory is an important mathematical tool for studying space motion. Torsor is composed of two three-dimensional vectors, which respectively represent the direction and position of vectors [19]. Furthermore, small displacement torsor is commonly used, which is a vector composed of weeny displacement generated by a rigid body with six motion components. In 1996, Bourdet et al. [20] first introduced small displacement torsor into the tolerance modeling field, and proposed to replace the variation of the actual tolerance surface in tolerance zone with the variation of small displacement torsor parameters. In the three-dimensional space, any physical quantity has three translational degrees of freedom along the coordinate axis and three rotational degrees of freedom around the coordinate axis, namely $d=(u, v, w)$ and $\theta=(\alpha, \beta, \gamma)$ are respectively the slightly changed vectors for the translation and rotation. The resultant vector of above mentioned two groups of vectors $D=d=(d, \theta)=(u, v, w, \alpha, \beta, \gamma)$ is called small displacement torsor, where $u, v, w, \alpha, \beta, \gamma$ is the small displacement torsor parameters.

In three-dimensional space, any physical object can be simply abstracted into the basic elements, namely points, lines and planes. Similarly, the geometric elements of the geometric error can be expressed by their respective small displacement torsors [21]. In theory, there are six torsor parameters for any three-dimensional characteristic surface, namely three 
translations and three rotations. However, in practical product processing, due to the existence of constraints, some torsor parameters of geometric elements with different types of geometric errors are taken as zero. The rule of setting the parameter as zero is : when the geometric element moves along a certain direction, its motion trajectory does not generate new error sweep entity. Ref. [22] listed several common torsor expressions corresponding to form and position errrors.

\subsection{Geometric error constraints of guide rail}

The kinematic error of the linear axis of machine tool is mainly manifested as the deviation between the actual and ideal position of rolling guide rail, which can be expressed by torsor parameters $(u, v, 0, \alpha, \beta, \gamma)$. According to the design requirements, neglecting the dimension error, the pose error of rolling guide rail is determined by the planeness error of the assembly main datum surface, the perpendicularity error and the parallelism error of the assembly subordinate datum surface. In this paper, they are called the geometric error of linear guide rail, as shown in Fig. 1.
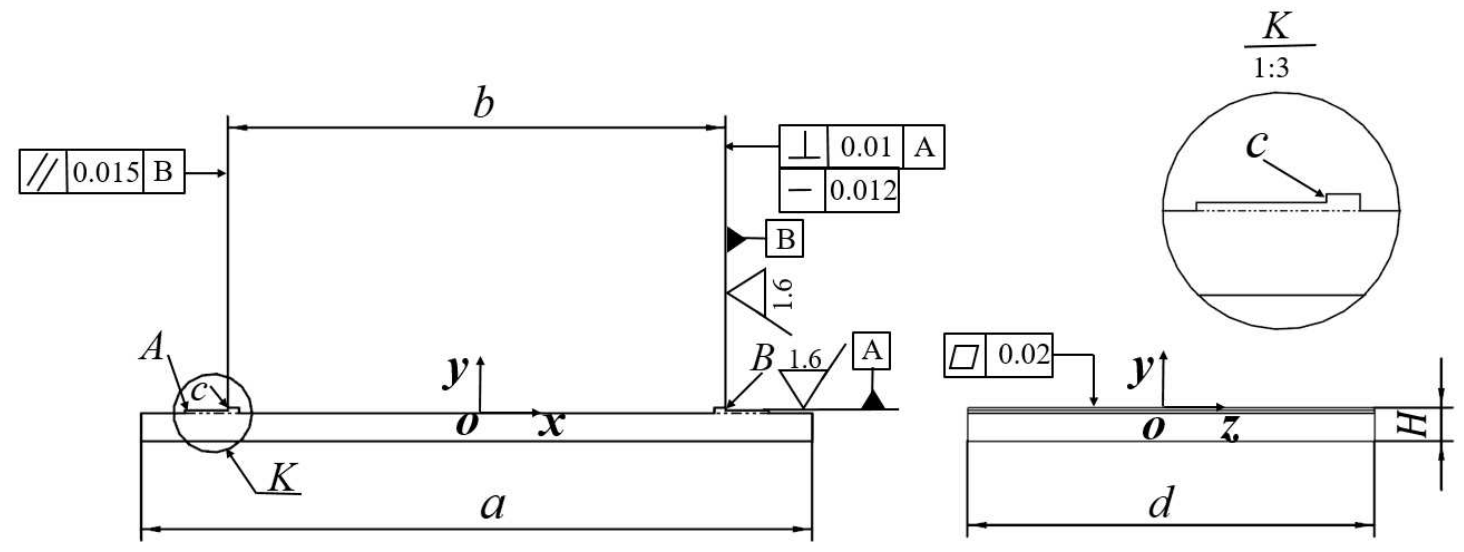

Fig. 1 Geometric errors of assembly surface of linear guide rail

\section{(1) flatness error}

The assembly datum surface of the guide rail usually has the main datum surface $A$ and two subordinate datum surface $B$ and $C$. The geometric errors (flatness, perpendicularity and parallelism) of the assembly surface of guide rail are shown in the Fig. 1. For the purpose of simplifying analysis, the coordinate system is set at the center of the main assembly datum 
surface. In the Fig. 2(a), the width and length of the assembly main datum surface $A$ are $a$ and $d$, and surface $C$ and $B$ are the subordinate datum surface for the left and right guide rail respectively, the height of the assembly main datum surface $A$ are $H$, the distance of surface $B$ and $C$ along $x$ axis is $b$. According to the design requirements, the flatness error of assembly main datum surface is $T_{a}$, the perpendicularity error of right datum surface B and A is $T_{b}$, and the parallelism error of left datum surface $C$ and $B$ is $T_{c}$. Assuming that the dimension tolerance is neglected, the flatness error of the assembly main datum surface $A$ is $T_{a}$, so the torsor model is expressed as $\left(0, v_{T}, 0, \alpha_{T}, 0, \delta_{T}\right)$, and the variation range constraints [23] of the torsor parameters are

$$
\left\{\begin{array}{l}
-T_{a} / 2 \leq v_{T} \leq T_{a} / 2 \\
-\frac{T_{a}}{d} \leq \alpha_{T} \leq \frac{T_{a}}{d} \\
-\frac{T_{a}}{a} \leq \delta_{T} \leq \frac{T_{a}}{a}
\end{array}\right.
$$

The variation range formulas of main assembly datum surface $A$ are

$$
\left\{\begin{array}{l}
y_{T}=v_{T}+x \cdot \delta_{T}+z \cdot \alpha_{T} \\
\Delta y_{T}=x \cdot \delta_{T}+z \cdot \alpha_{T}
\end{array}\right.
$$

where $\Delta y_{T}$ represents the distance between any two points on the surface. The length and width of rectangular surface are limited within the flatness error $T_{a}$, the extreme value occurs at the vertex of the rectangular surface. Therefore, the torsor parameter constraint formulas of flatness error of assembly main datum surface are expressed as

$$
\left\{\begin{array}{l}
-T_{a} / 2 \leq v_{T}+x \cdot \delta_{T}+z \cdot \alpha_{T} \leq T_{a} / 2 \\
-T_{a} / 2 \leq x \cdot \delta_{T}+z \cdot \alpha_{T} \leq T_{a} / 2
\end{array}\right.
$$

where $x, z$ value is the four limit positions of surface $A$, that is four corners on the assembly main datum surface $A$ in the figure, namely the setting range of $x$ value is $(-a / 2, a / 2)$, and the setting range of $z$ value is $(-d / 2, d / 2)$. 


\section{(2) parallelism error}

According to the design requirements, the assembly subordinate datum surface $C$ of the left guide rail is required to have a parallelism error $T_{c}$ with the assembly main datum surface $A$, furthermore the corresponding small displacement torsor model is $\left(u_{z}, 0,0,0, \beta_{z}, \delta_{z}\right)$, and the variation range constraints [23] are

$$
\left\{\begin{array}{c}
-\frac{T_{c}}{2} \leq u_{z} \leq \frac{T_{c}}{2} \\
-\frac{T_{c}}{d} \leq \beta_{z} \leq \frac{T_{c}}{d} \\
-\frac{T_{c}}{H} \leq \delta_{z} \leq \frac{T_{c}}{H}
\end{array}\right.
$$

Similar to the constraints form of flatness error, the torsor parameters constraints of parallelism error are

$$
\left\{\begin{array}{l}
-\frac{T_{c}}{2} \leq y \cdot \delta_{z}+z \cdot \beta_{z} \leq \frac{T_{c}}{2} \\
-\frac{T_{c}}{2} \leq u_{z}+y \cdot \delta_{z}+z \cdot \beta_{z} \leq \frac{T_{c}}{2}
\end{array}\right.
$$

where $y, z$ value is the four limit positions of surface $C$, that is four vertices on the assembly main datum surface $C$ in the figure, namely the setting range of $y$ value is $\mid(0, H)$, and the setting range of $z$ value is $(-d / 2, d / 2)$, as shown in Fig. 2 (b).

\section{(3) perpendicularity error}

Similar to the subordinate datum surface $C$ of left guide rail, according to the design requirements, the assembly subordinate datum surface B of the right guide rail is required to have a perpendicularity error $T_{c}$ with the assembly main datum surface $A$, moreover the corresponding small displacement torsor model is $\left(u_{Y}, 0,0,0, \beta_{Y}, \delta_{Y}\right)$, and the variation range constraints [23] are 


$$
\left\{\begin{array}{l}
-\frac{T_{b}}{2} \leq u_{Y} \leq \frac{T_{b}}{2} \\
-\frac{T_{b}}{d} \leq \beta_{Y} \leq \frac{T_{b}}{d} \\
-\frac{T_{b}}{H} \leq \delta_{Y} \leq \frac{T_{b}}{H}
\end{array}\right.
$$

Similar to the constraints form of parallelism error, the torsor parameters constraints of perpendicularity error are

$$
\left\{\begin{array}{l}
-\frac{T_{b}}{2} \leq y \cdot \delta_{Y}+z \cdot \beta_{Y} \leq \frac{T_{b}}{2} \\
-\frac{T_{b}}{2} \leq u_{Y}+y \cdot \delta_{Y}+z \cdot \beta_{Y} \leq \frac{T_{b}}{2}
\end{array}\right.
$$

where $y, z$ value is the four limit positions of surface $B$, that is four corners on the assembly subordinate datum surface $B$ in the figure, namely the setting range of $y$ value is $(-d / 2, d / 2)$, and the setting range of $z$ value is $(0, H)$, as shown in Fig. 2 (c).

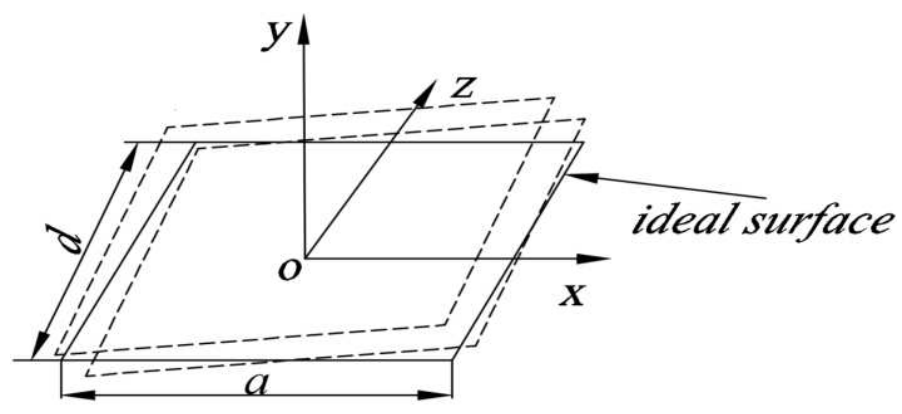

(a)

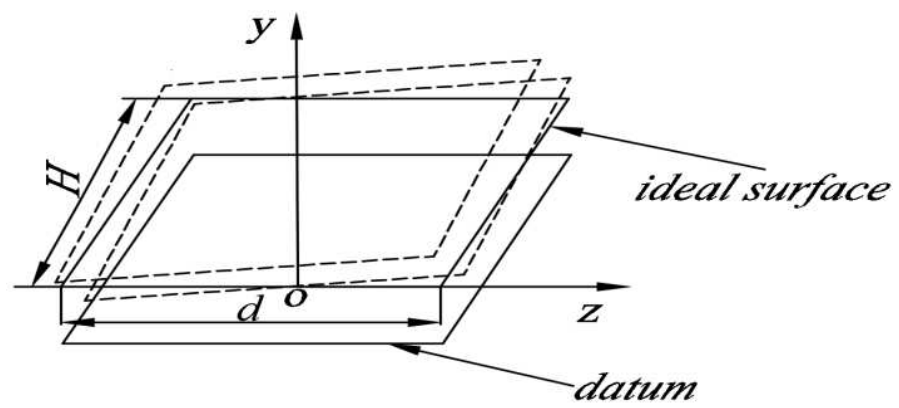

(b) 


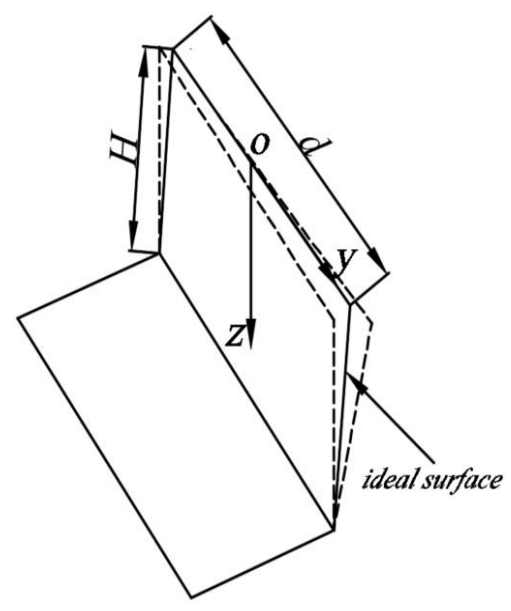

(c)

Fig. 2 Variation range (a) flatness; (b) parallelism; (c) perpendicularity

\subsection{MCS method}

MCS is a method to solve approximate solutions of problems through statistical tests and stochastic simulation of random variables [24]. It has many advantages such as strong adaptability, simple calculation method, and solving errors independent of problem dimensions. With the MCS method, small displacement torsor parameters of geometric error are sampled, the sampling flow chart of MCS simulation is shown in Fig. 3. The generated corresponding random parameters are utilized to simulate flatness and parallelism and perpendicularity error, and then these random numbers meeting the constraint conditions are retained, and in contrast unsatisfactory ones are removed, finally above process are regarded as a basis of solving the kinematic error of the linear axis of machine tool. Taking the flatness error of linear guide rail's main assembly datum surface $A$ as an example, the steps to simulate the actual variation range of flatness error by MCS are as follows:

(1) Determine an ideal probability distribution model of geometric error torsor parameters. In general, the geometric error distribution of parts manufactured by mechanical processing conforms to the law of normal distribution [25], and its probability density function is assumed to be

$$
\phi(x)=\frac{1}{\sigma \sqrt{2 \pi}} \exp \left[\frac{-(x-u)^{2}}{2 \sigma^{2}}\right] \quad(-\infty<x<+\infty, \sigma>0)
$$


(2) Determine the mean value and variance of ideal probability distribution for torsor parameters of geometric error. The calculation results show that the probability of the value $x$ ranging from $u-3 \sigma$ to $u+3 \sigma$ is $99.73 \%$. From the perspective of engineering, it is generally considered that the distribution range of the normal distribution is $\pm 3 \sigma$. According to the torsor variation in equation (1), the mean and variance of the ideal distribution of torsor parameters $v_{T}, \alpha_{T}, \delta_{T}$ are $\left(0, T_{a} / 6\right),\left(0, T_{a} / 3 d\right),\left(0, T_{a} / 3 a\right)$, respectively. Similarly, the mean and variance of the torsor parameters $u_{z}, \beta_{z}, \delta_{z}$ of parallelism error are respectively $\left(0, T_{c} / 6\right),\left(0, T_{c} / 3 d\right),\left(0, T_{c} / 3 H\right)$, and the torsor parameters $u_{Y}, \beta_{Y}, \delta_{Y}$ of perpendicularity error are $\left(0, T_{b} / 6\right),\left(0, T_{b} / 3 d\right),\left(0, T_{b} / 3 H\right)$, respectively.

(3) According to the constraint requirements, the torsor parameters of geometric errors are sampled, and the total samples number in this paper is 10000 .

Similarly, both torsor parameters $\left(u_{z}, 0,0,0, \beta_{z}, \delta_{z}\right)$ samples of parallelism error and torsor parameters $\left(u_{Y}, 0,0,0, \beta_{Y}, \delta_{Y}\right)$ samples of perpendicularity error are also obtained. 


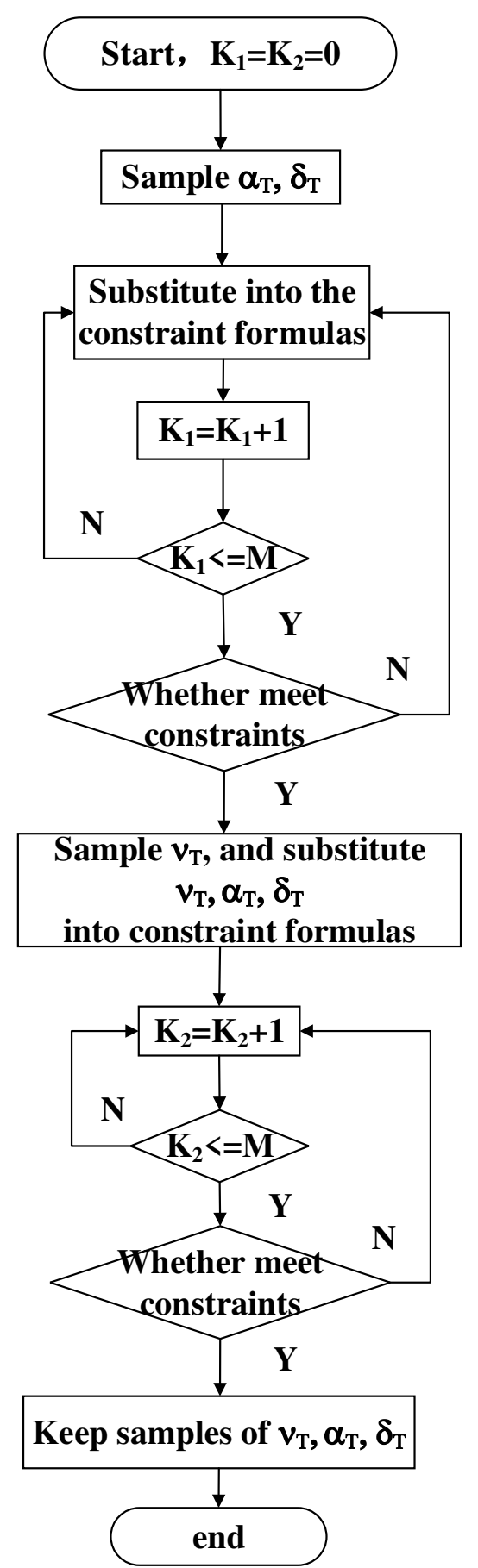

Fig. 3 Sampling flow chart of MCS simulation

\subsection{Geometric error simulation of guide rail}

The structure of the linear axis contains the base, linear guide rail and carriage in Fig. 4. The kinematic error of the linear axis is determined by the combined pose error, composed by the assembly main datum surface and subordinate datum surface of left and right guide rails. According to the master-slave relation of the assembly guide rails, the right guide rail is taken 
as the datum one, the torsor parameters of pose error for left and right guide rail are respectively $\left(u_{z}, v_{T}, 0, \alpha_{T}, \beta_{z}, \delta_{T}\right)$ and $\left(u_{Y}, v_{T}, 0, \alpha_{T}, \beta_{Y}, \delta_{T}\right)$.

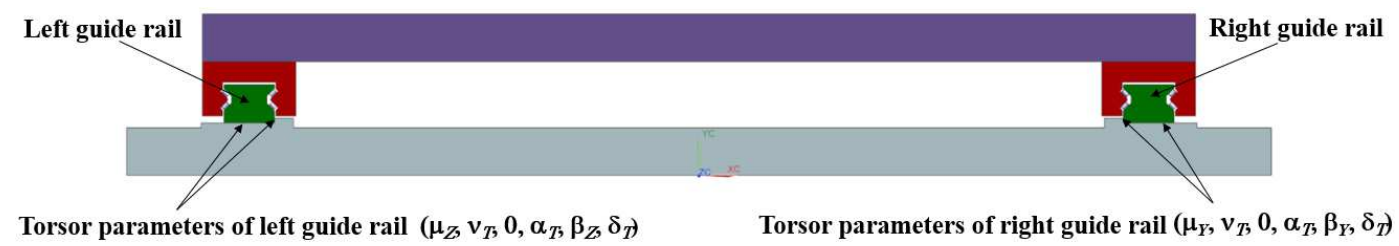

Fig. 4 Structure sketch of the linear axis

According to mentioned contents in above sections, torsor parameter of flatness error is $\left(0, v_{T}, 0, \alpha_{T}, 0, \delta_{T}\right)$, torsor parameter of parallelism error is $\left(u_{z}, 0,0,0, \beta_{z}, \delta_{z}\right)$, torsor parameter of comprehensive pose error for left guide rail is $\left(u_{z}, v_{T}, 0, \alpha_{T}, \beta_{z}, \delta_{T}\right)$. The simulation results by MCS method is shown in Fig. 5 .

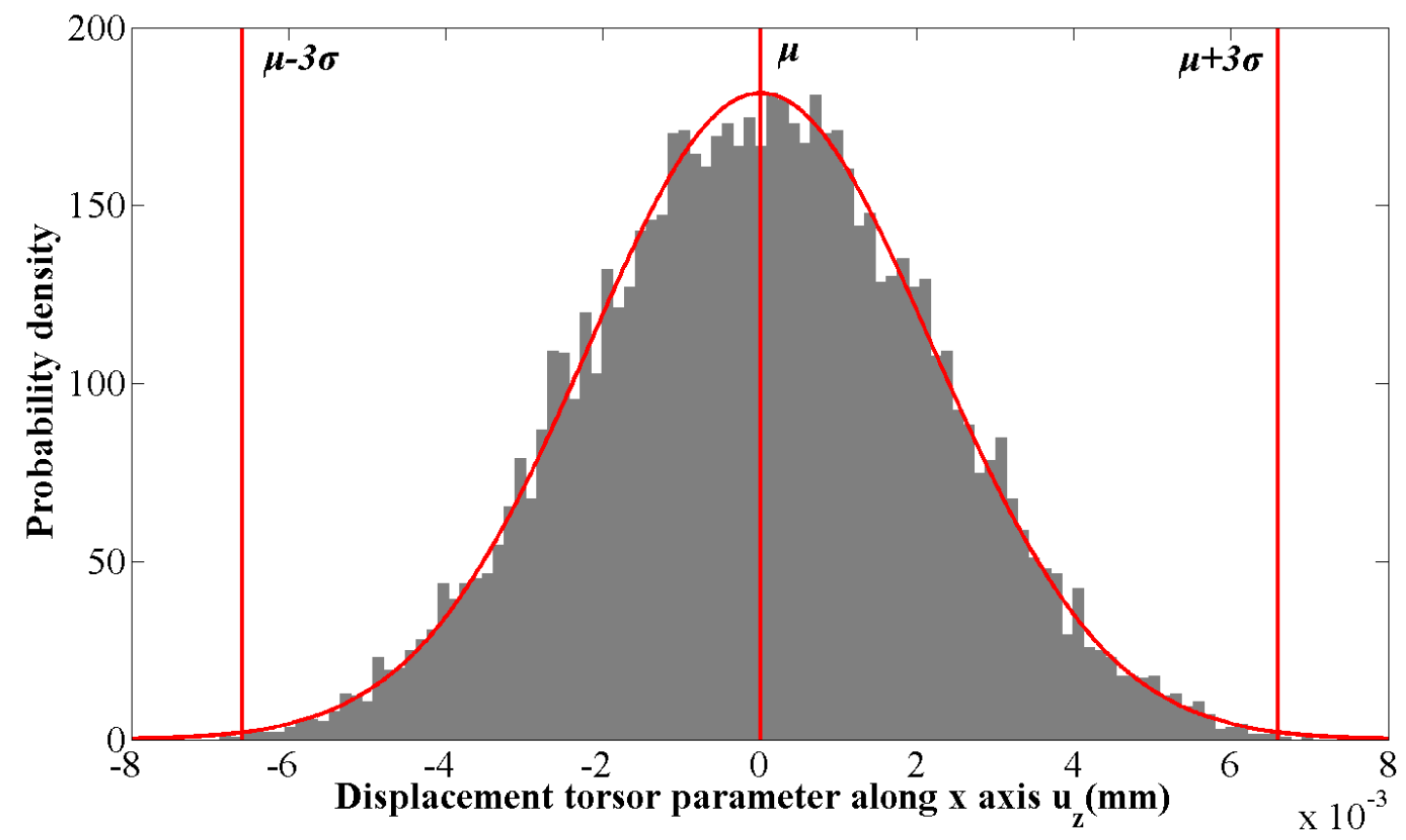

(a) 


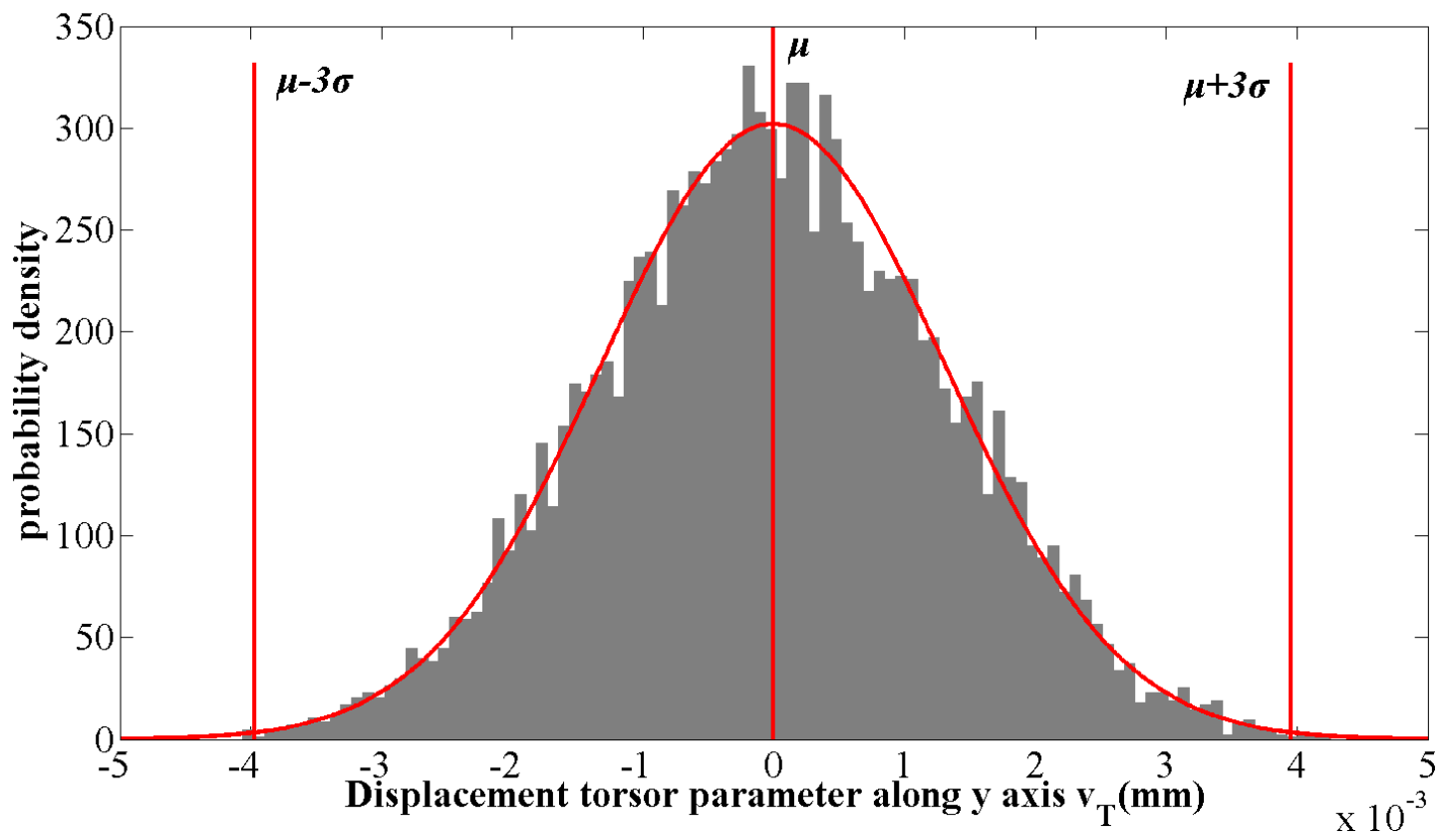

(b)

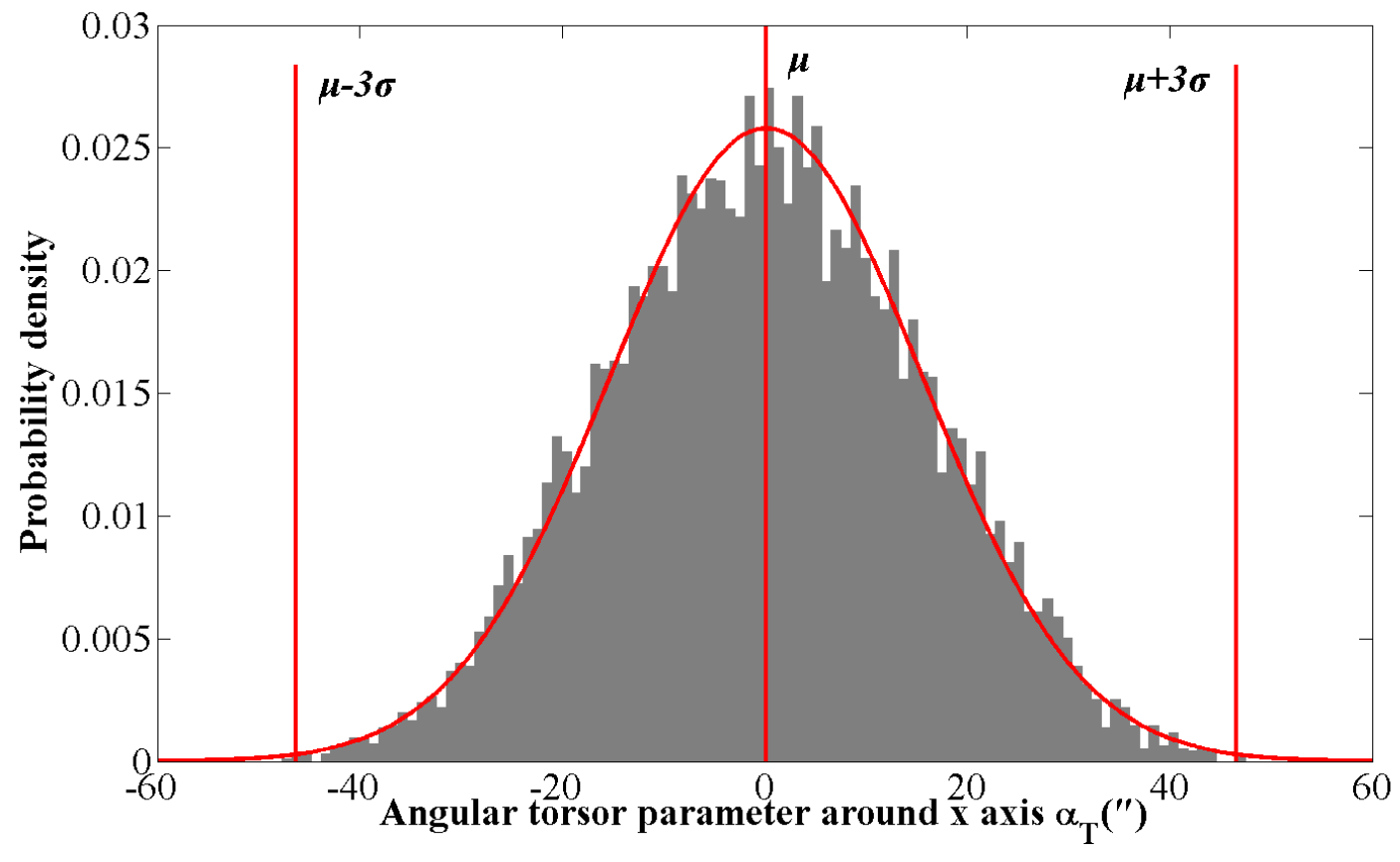

(c) 


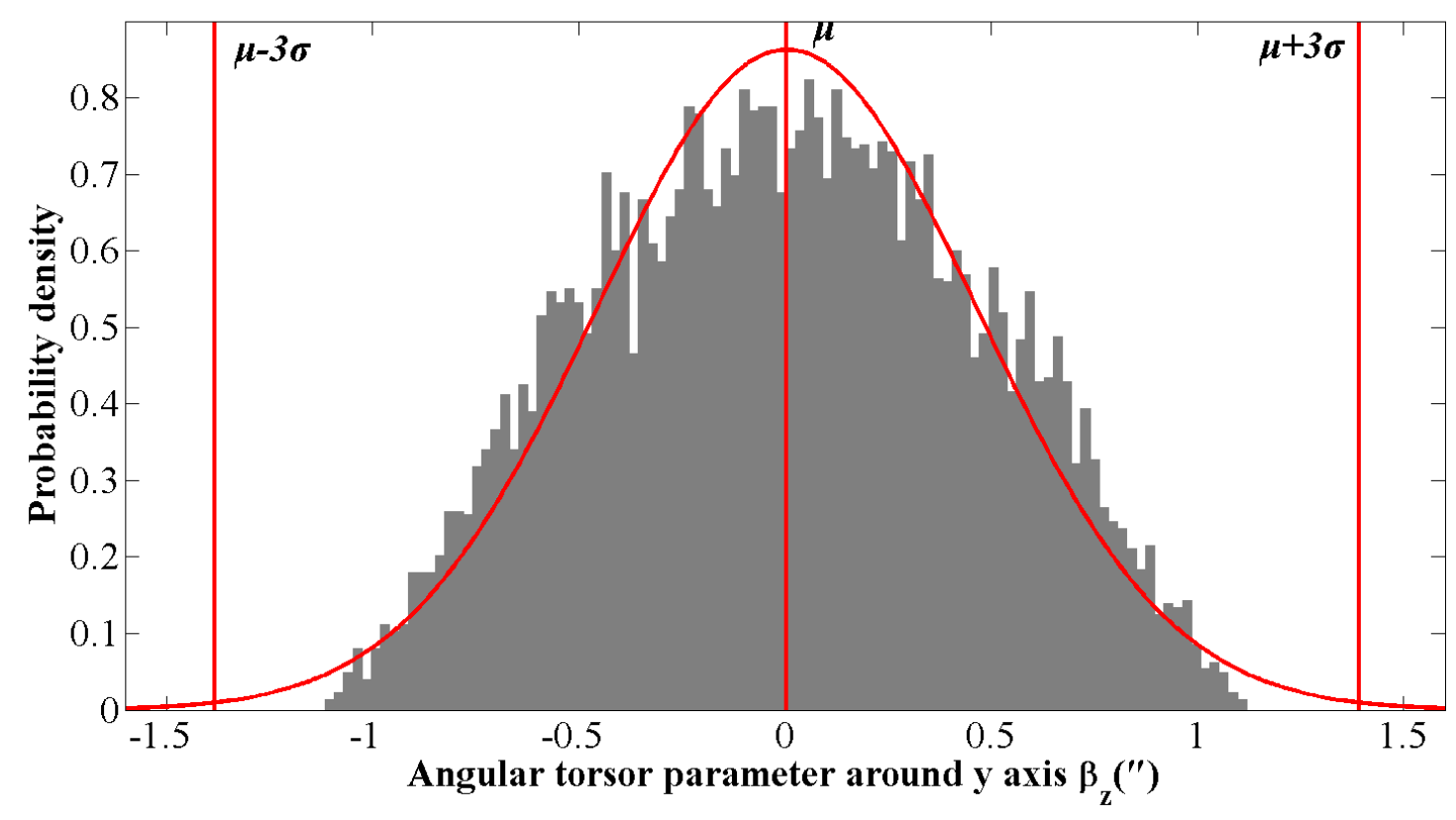

(d)

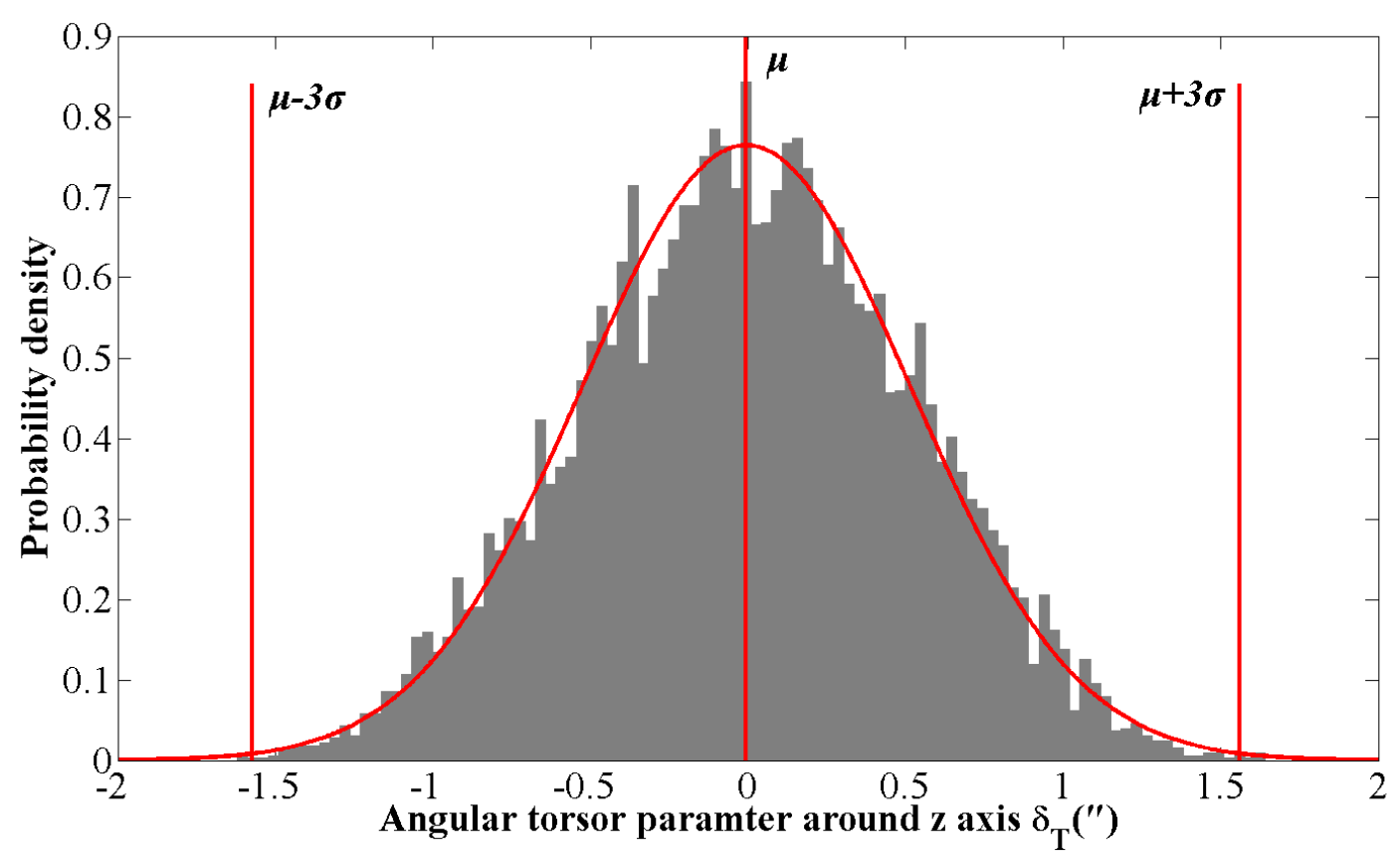

(e)

Fig. 5 MCS results of comprehensive pose error for left guide rail

Similarly, torsor parameter of flatness error is also $\left(0, v_{T}, 0, \alpha_{T}, 0, \delta_{T}\right)$, torsor parameter of perpendicularity error is $\left(u_{Y}, 0,0,0, \beta_{Y}, \delta_{Y}\right)$, the torsor parameter of comprehensive pose error for right guide rail is $\left(u_{Y}, v_{T}, 0, \alpha_{T}, \beta_{Y}, \delta_{T}\right)$. The simulation results by MCS method is 
shown in Fig. 6.

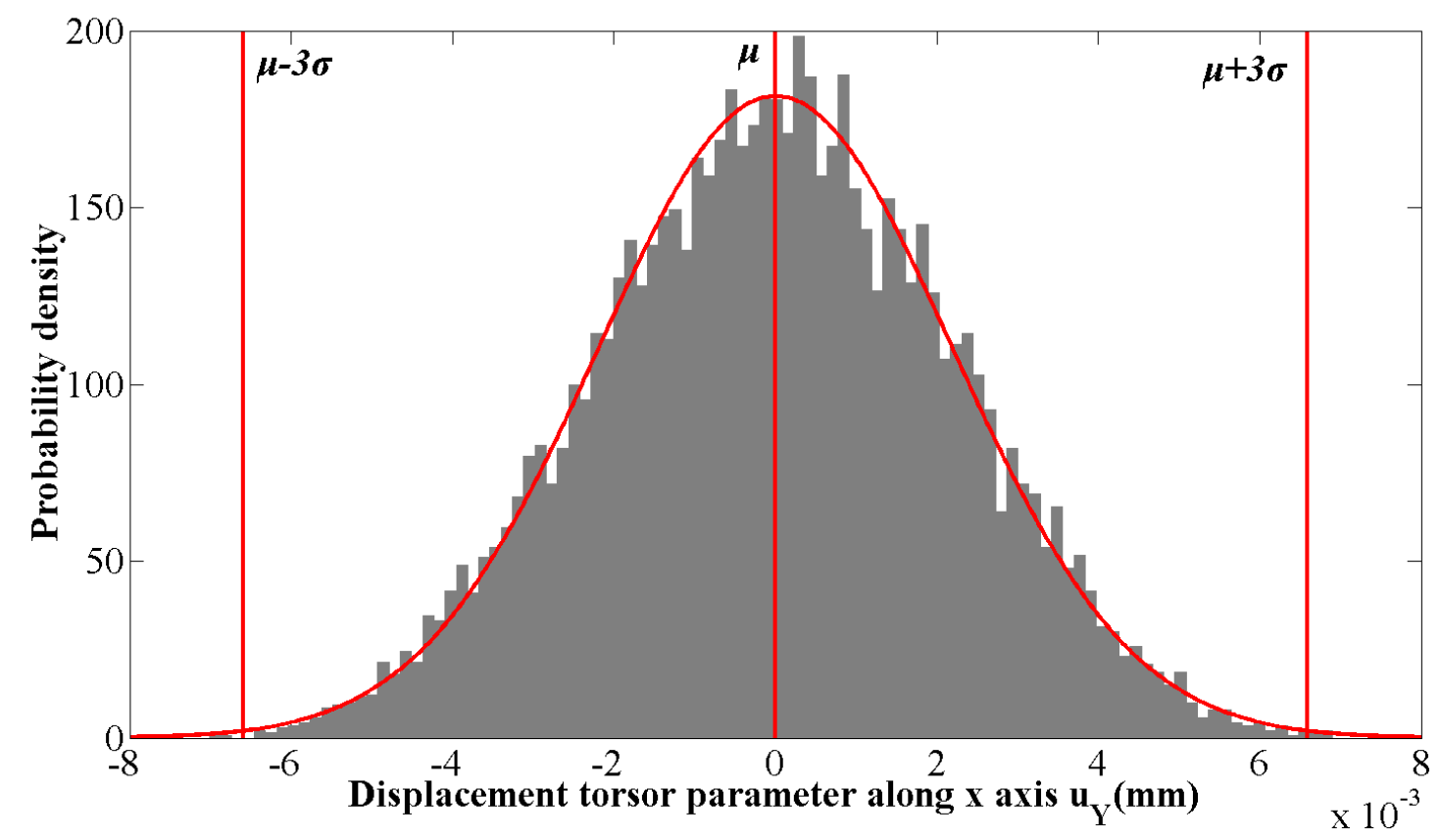

(a)

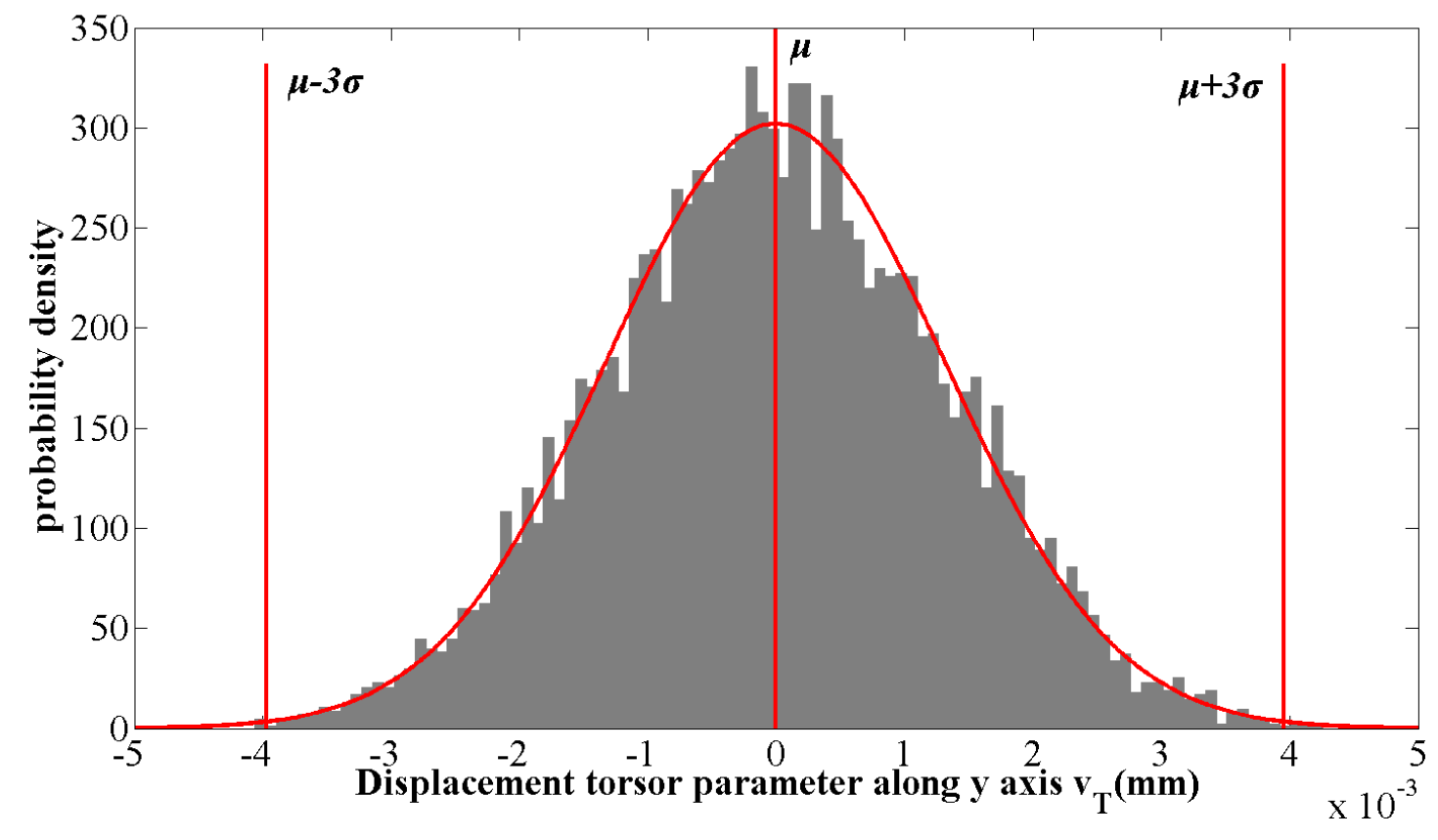

(b) 


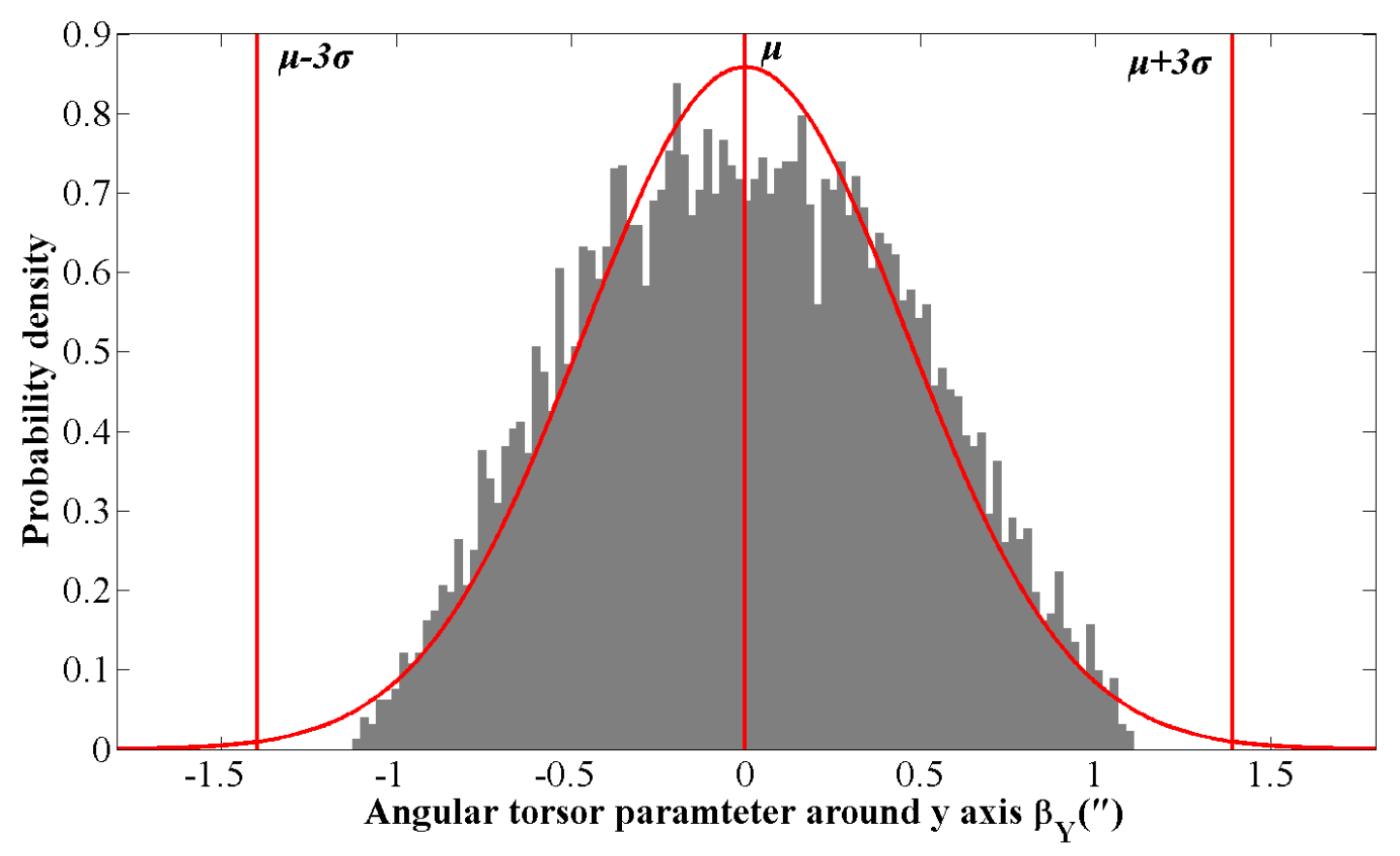

(c)

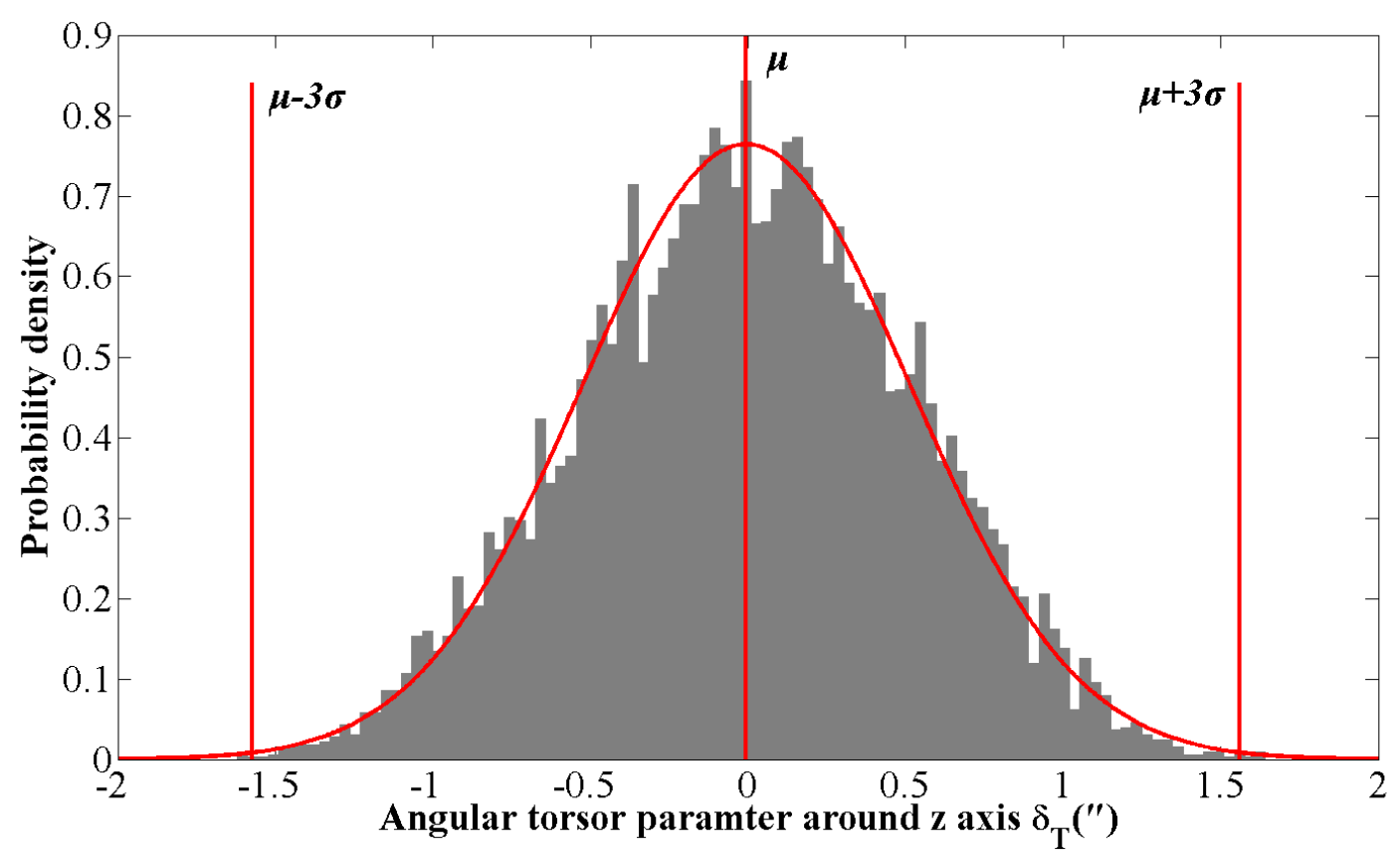

(d) 


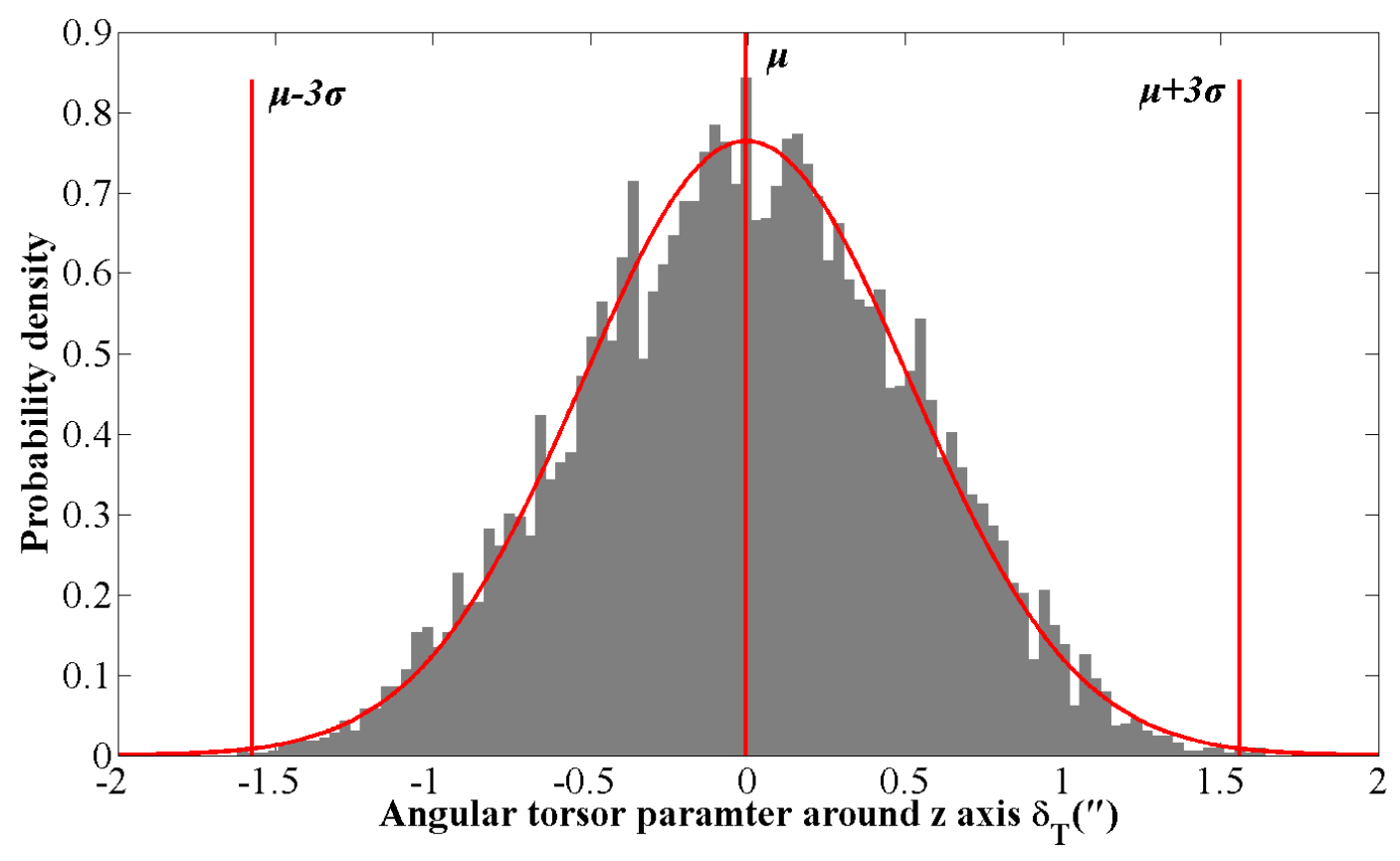

(e)

Fig. 6 MCS simulation results of comprehensive pose error for left guide rail

\section{Error equivalence model of linear axis}

Section 3 consists of two parts: kinematic error model of a single slider and kinematic error model of the linear axis. Firstly, the kinematic error of a single slider are calculated, and then kinematic error of the linear axis can be obtained through the principle of vector summation. Finally, as for the kinematic error solution process of linear axis, the flow chart of technical route is shown in Fig. 7, as follows. 


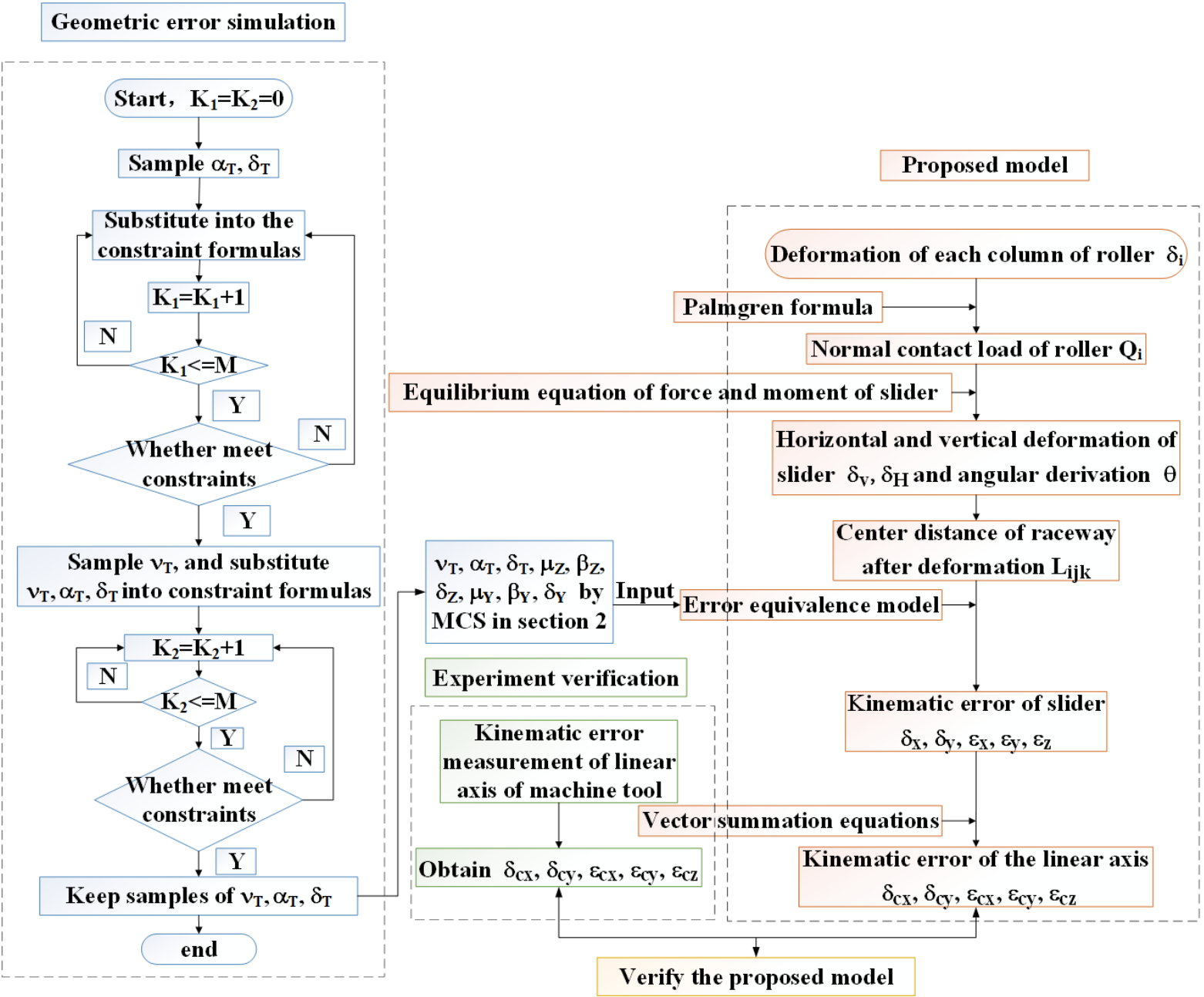

Fig. 7 Flow chart of technical route

\subsection{Error equivalence model of a single slider}

In order to obtain the kinematic error of the linear axis of machine tool, the kinematic error of a single slider is firstly calculated. Anytime, the linear guide rail keep balance under the load namely itself gravity and the elastic restoring force of rollers. Moreover, the geometric error of guide rail and the position error of slider will influence the contact condition among the slider, guide rail and rollers in Fig. 8. Consequently, the elastic deformation of rollers can be effected. Hence, in this paper, the error equivalence model is proposed, namely the geometric error of guide rail and the position error of slider are equivalent to the elastic deformation of rollers. Besides, the Hertz contact theory is adopted to construct the static balance equations of linear guide rail. Consequently, the kinematic error of linear guide rail based on the error equivalence model is obtained. 


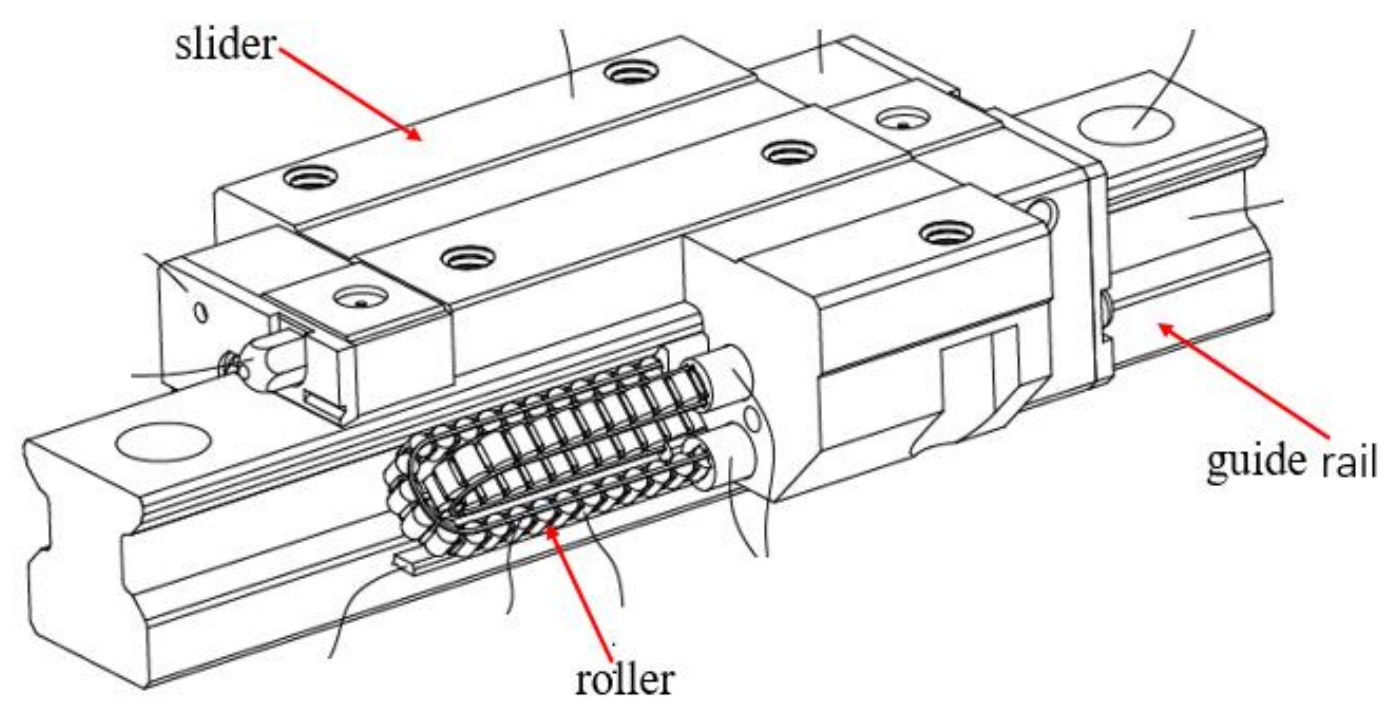

Fig. 8 Structure model of linear guide rail

The positioning accuracy of linear axis of the machine tool is mainly determined by the driving unit and the control unit. When the motion system of the linear axis moves along $z$ axis, there occurs six kinematic errors, the positioning accuracy is neglected, including two translational errors ( $\Delta y$ and $\Delta x)$ along $y$ and $x$ axis, and three rotational errors $(\Delta u, \Delta v, \Delta w)$ around $x, y$ and $z$ axis, as is shown in Fig. 9. Furthermore, the kinematic errors of these five degrees of freedom of the machine tool mainly derive from the straightness of the guide rail. Hence, in this paper, five items of kinematic errors, namely the straightness along $x$ and $y$ axis ( $\Delta y$ and $\Delta x$ ), and angular errors of pitch, yaw and roll $(\Delta u, \Delta v, \Delta w)$, the physical meaning of each error is listed in Table 1.

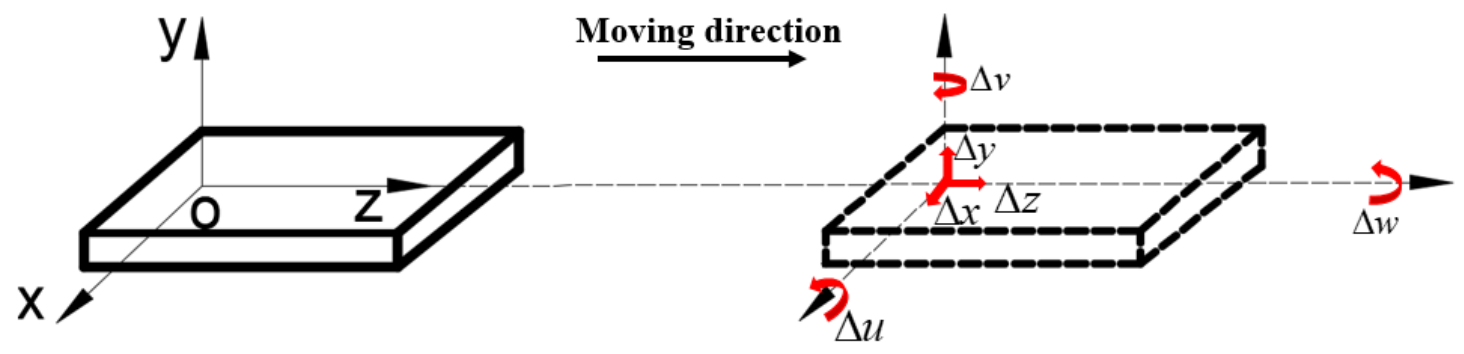

Fig. 9 Six directional errors of the linear axis 
Table 1 Physical meanings of five kinematic errors in the linear axis (moving along $z$ axis)

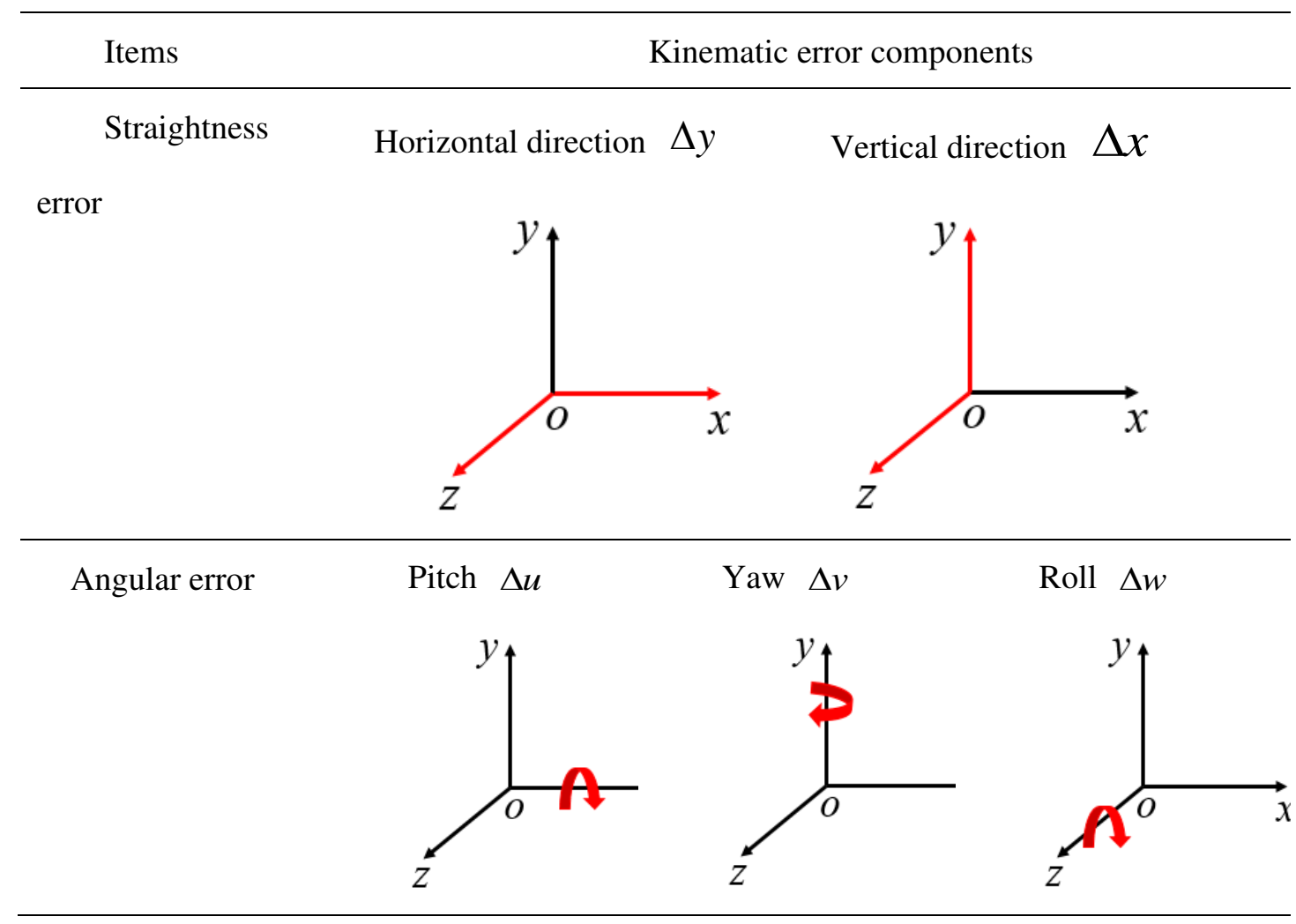

According to the practical load condition of linear guide rail in milling machining, it can be decomposed into the normal load $F_{v i}$ on the upper surface of slider, the lateral load $F_{H i}$ perpendicular to the side surface of slider and the rotational torque $M_{z}$ around $z$ axis of translational direction. Assuming that four columns of rollers are denoted by the variables, $i=$ 1, 2, 3, 4 in Fig. 10, the coordinate system $o-x y z$ is established at the symmetrical center point $o$ of rollers. As for each roller in ith column, the normal load from the slider contact surface is $Q_{i}$, moreover the initial deformation of each column of roller under the preload is $\delta_{0}$, the elastic deformation of the ith column of rollers is $\delta_{i}$. The center distance between two column of rollers are $l_{x}$ and $l_{y}$ along $x$ and $y$ axis, respectively. The angle between the rollers and raceway is $\alpha$. Therefore, the displacement of slider under load in $x$ and $y$ axis are denoted as $\delta_{V}$ and $\delta_{H}$, the angle around $z$ axis under rotational torque is $\theta$. As a result, 
considering the structure characteristics of linear guide rail, the elastic deformation of the ith column of rollers is expressed as in Eq. (9).

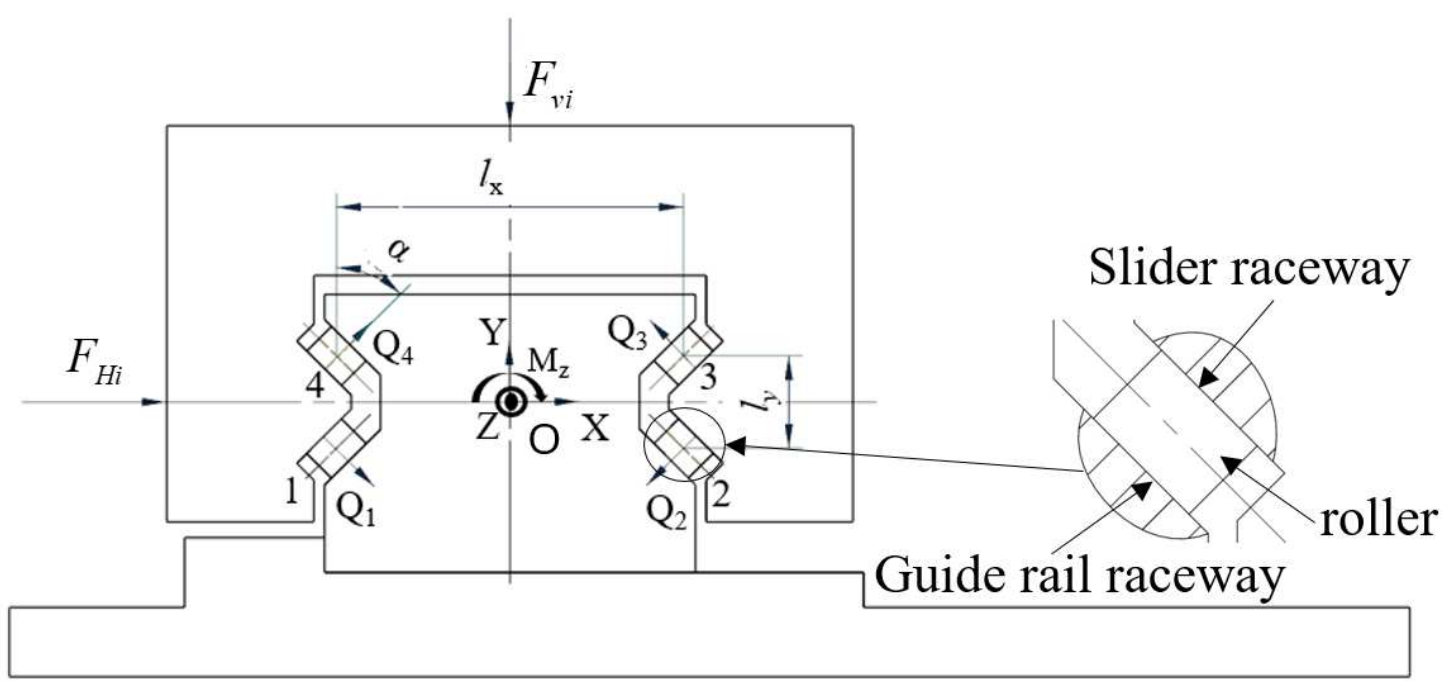

Fig. 10 Load analysis of single guide rail

$$
\left\{\begin{array}{l}
\delta_{1}=\delta_{0}+\delta_{v} \cdot \cos \alpha+\delta_{H} \cdot \sin \alpha-\frac{\theta \cdot\left(l_{x} \cdot \cos \alpha+l_{y} \cdot \sin \alpha\right)}{2} \\
\delta_{2}=\delta_{0}+\delta_{v} \cdot \cos \alpha-\delta_{H} \cdot \sin \alpha+\frac{\theta \cdot\left(l_{x} \cdot \cos \alpha+l_{y} \cdot \sin \alpha\right)}{2} \\
\delta_{3}=\delta_{0}-\delta_{v} \cdot \cos \alpha-\delta_{H} \cdot \sin \alpha-\frac{\theta \cdot\left(l_{x} \cdot \cos \alpha+l_{y} \cdot \sin \alpha\right)}{2} \\
\delta_{4}=\delta_{0}-\delta_{v} \cdot \cos \alpha+\delta_{H} \cdot \sin \alpha+\frac{\theta \cdot\left(l_{x} \cdot \cos \alpha+l_{y} \cdot \sin \alpha\right)}{2}
\end{array}\right.
$$

When the slider is under the loads, the elastic deformation occurs between rollers and raceway. Since the width of the contact region between roller and raceway is far less than the curvature radius of contact point, it can be equivalent to the contact problem between an elastic cylindrical and a rigid plane [26]. Therefore, as for the motion of a finite length roller in the raceway, the relationship between the elastic deformation and load can be obtained by the Palmgren empirical formula [27], as shown in Eq. (10).

$$
\delta_{i}=1.36 \times\left(\frac{\left(1-v_{1}\right)^{2}}{E_{1}}+\frac{\left(1-v_{2}\right)^{2}}{E_{2}}\right)^{0.9} \times \frac{Q_{i}^{0.9}}{l_{e}^{0.8}}
$$

where the constant $E_{1}, E_{2}, v_{1}$ and $v_{2}$ are the elastic modulus and Poisson's ratio of two contact bodies, respectively. $l_{e}$ represents the effective length of roller. According to Eq. 
(10), for the elastic deformation $\delta_{i}$ of the ith column of rollers, when $\delta_{i} \leq 0$, the initial deformation of the ith column of rollers disappears under the preload. When $\delta_{i}>0$, there occurs a deformation value for rollers, so the normal load of contact surface on each column of roller is written as in Eq. (11).

$$
Q_{i}= \begin{cases}\left(\frac{\delta_{i} \cdot l_{e}^{0.8}}{3.84 \times 10^{-5}}\right)^{10 / 9}, & \delta_{i}>0 \\ 0 \quad & \delta_{i} \leq 0\end{cases}
$$

For single linear guide rail and slider, force and moment keep balance at the coordinate origin $O$ of guide rail, so Eq. (12) are obtained. In addition, there are three assumptions in the proposed model: (1) the friction between roller and raceway is ignored; (2) the normal contact forces of all rollers in each raceway of a single slider are equal; (3) The center line between the raceway of guide rail and slider is parallel before and after rollers deformation. The load analysis of single guide rail and slider is shown in Fig. 12.

$$
\begin{aligned}
& \left\{\begin{array}{l}
\left(Q_{1} \cdot \cos \alpha+Q_{2} \cdot \cos \alpha-Q_{3} \cdot \cos \alpha-Q_{4} \cdot \cos \alpha\right) \cdot n_{e}=F_{v i} \\
\left(Q_{1} \cdot \sin \alpha-Q_{2} \cdot \sin \alpha-Q_{3} \cdot \sin \alpha+Q_{4} \cdot \sin \alpha\right) \cdot n_{e}=F_{H i} \\
{\left[\begin{array}{l}
\frac{Q_{1} \cdot\left(l_{x} \cdot \cos \alpha+l_{y} \cdot \sin \alpha\right)}{2}-\frac{Q_{2} \cdot\left(l_{x} \cdot \cos \alpha+l_{y} \cdot \sin \alpha\right)}{2} \\
\left.+\frac{Q_{3} \cdot\left(l_{x} \cdot \cos \alpha+l_{y} \cdot \sin \alpha\right)}{2}-\frac{Q_{4} \cdot\left(l_{x} \cdot \cos \alpha+l_{y} \cdot \sin \alpha\right)}{2}\right] \cdot n_{e}=M_{z}
\end{array}\right.}
\end{array}\right. \\
& (i=1,2,3,4)
\end{aligned}
$$

where $n_{e}$ denotes the number of effective rollers subject to load in each column. The normal force of contact surface calculated by Eq. (11) is substituted into Eq. (12), so the relationships between the normal load $F_{v i}$, the side load $F_{H i}$, rotational moment $M_{z}$ and the displacement $\delta_{v}$ along $x$ axis , the displacement along $y$ axis $\delta_{H}$, the angle $\theta$ around $z$ axis is written as 


$$
\left\{\begin{array}{l}
\delta_{V}=f_{1}\left(F_{V i}, F_{H i}, M_{Z}\right) \\
\delta_{H}=f_{2}\left(F_{V i}, F_{H i}, M_{Z}\right) \\
\theta=f_{3}\left(F_{V i}, F_{H i}, M_{Z}\right)
\end{array}\right.
$$

where $f_{1}, f_{2}, f_{3}$ are the three different functions, respectively.

After the deformation and rotation angle of a single slider are obtained, the kinematic error of a single slider is calculated by the error equivalent model of linear guide rail. The geometric error of guide rail and the pose error of slider are equivalent to the elastic deformation of roller. The geometric error of guide rail is reflected as the offset of guide rail raceway center, and the pose error of slider is reflected as the offset of slider raceway center. Hence, the offset of the center distance of guide rail raceway and slider raceway is equivalent to the elastic deformation of roller. Ideally, the contact model of rollers before and after elastic deformation is shown in Fig. 11.

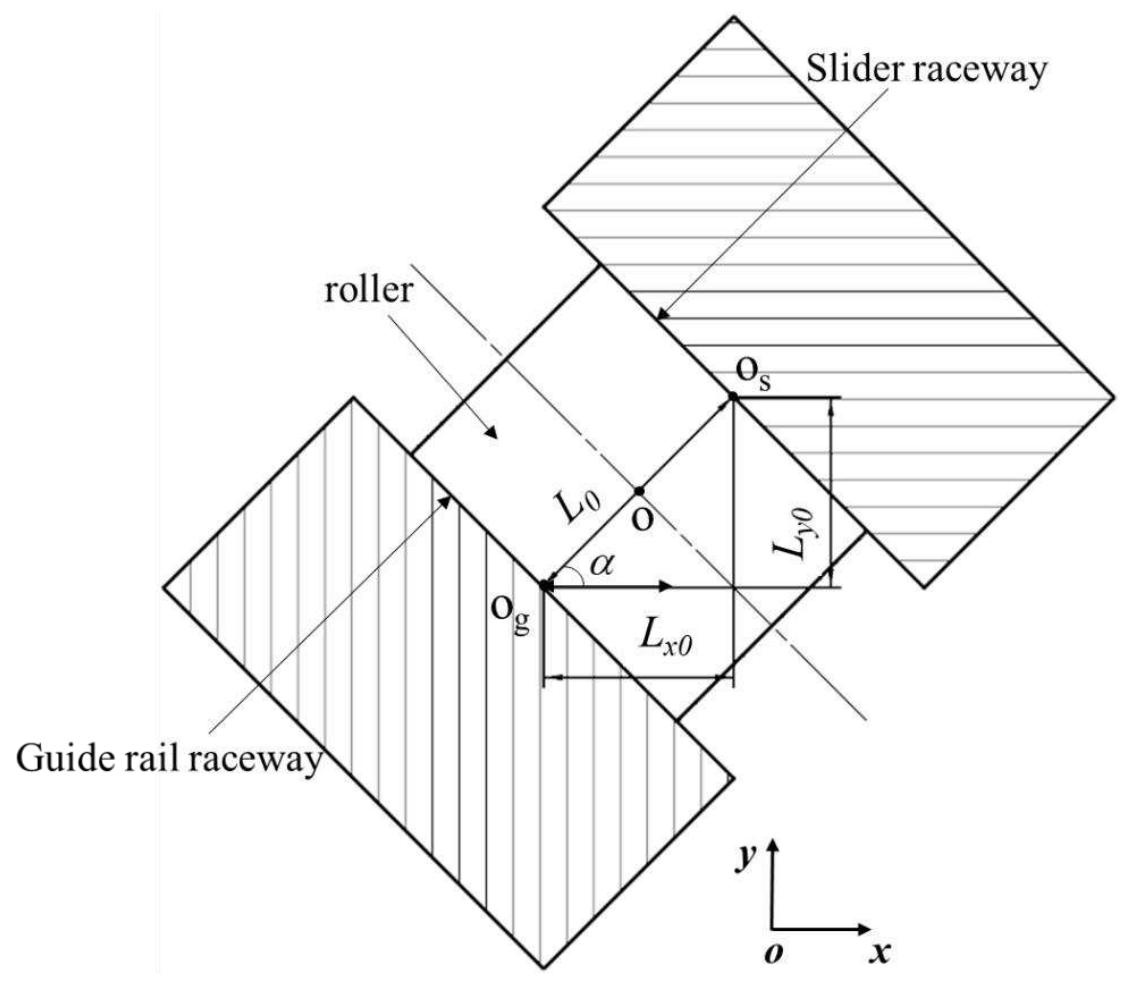

(a) 


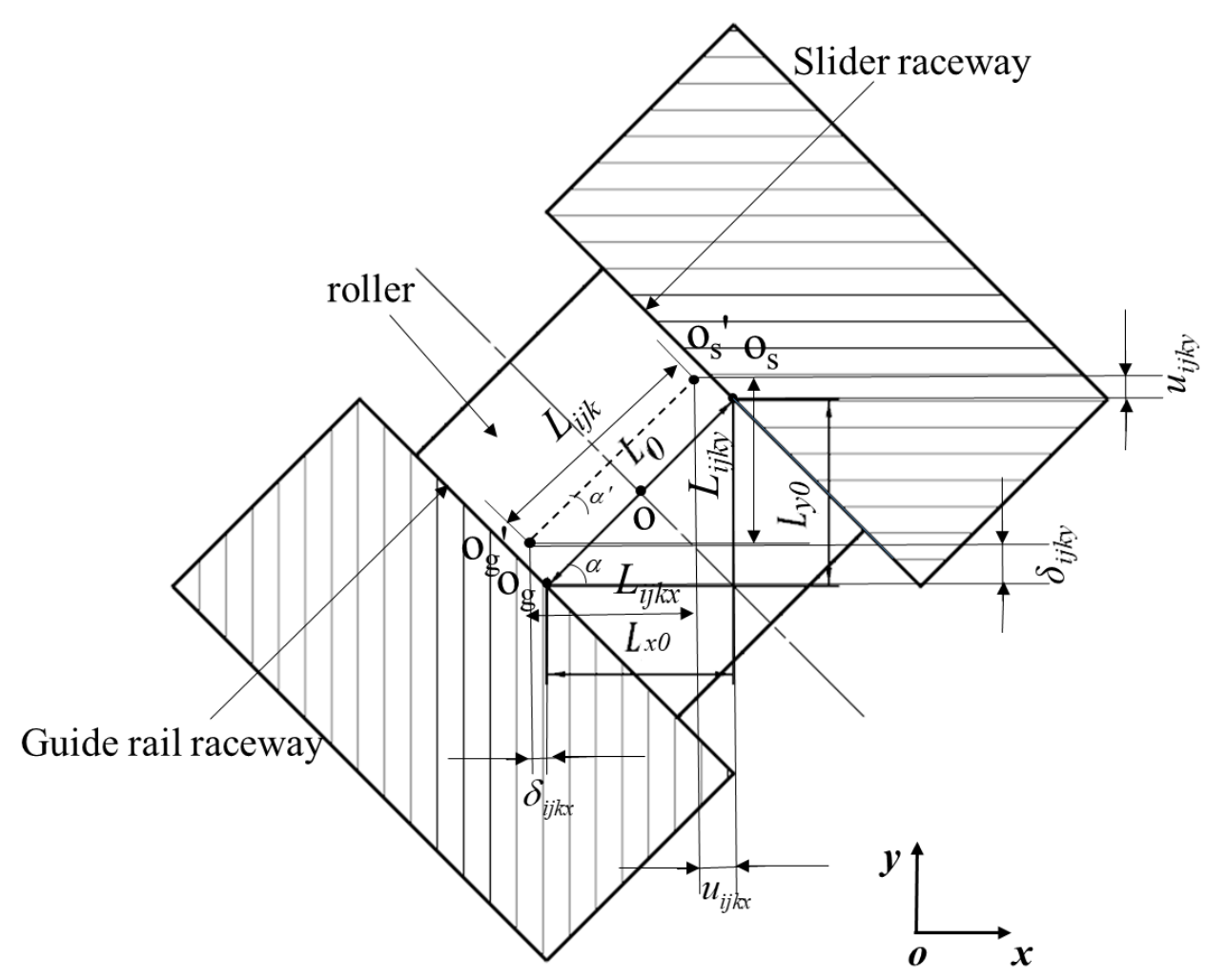

(b)

Fig. 11 (a) Contact model of linear guide rail before deformation;

(b) Error equivalence model after deformation

In Fig. 11 (a), $o$ is the geometric center of roller, $\alpha$ is the angle between the center line of roller and raceway and horizontal direction before deformation. $o_{g}$ and $o_{s}$ are the curvature center of guide rail and slider before deformation, $L_{o}$ is the distance of raceway center between guide rail and slider before deformation, $L_{x o}$ and $L_{y o}$ are the components in $x$ and $y$ direction in Eq. (14), respectively. In Fig. 11 (b), $\alpha^{\prime}$ is the angle between the center line of roller and raceway and horizontal direction after deformation. $o_{g}{ }^{\prime}$ and $o_{s}{ }^{\prime}$ are the curvature center of guide rail and slider after deformation, $\delta_{i j k x}$ and $\delta_{i j k y}$ are the horizontal and vertical offset of the actual curvature center of the guide rail raceway after deformation, $u_{i j k x}$ and $u_{i j k y}$ are the horizontal and vertical offset of the actual curvature center of the slider after deformation, $L_{i j k}$ is the distance of raceway center between guide 
rail and slider after deformation, $L_{i j k x}$ and $L_{i j k y}$ are the components in $x$ and $y$ direction in Eq. (16), respectively.

$$
\left\{\begin{array}{l}
L_{x 0}=D_{a} \times \cos \alpha \\
L_{y 0}=D_{a} \times \sin \alpha
\end{array}\right.
$$

where $D_{a}$ is the diameter of roller before deformation. On the basis of the deformation of slider in Eq. (13), the distance of raceway center between guide rail and slider after deformation is calculated by Eq. (15).

$$
\Delta=\sqrt{\left(\delta_{v}^{2}+\delta_{H}^{2}\right)}
$$

Then Eq. (15) is submitted into Eq. (16), so the center distance $L_{i j k}$ between guide rail raceway and slider raceway is deduced in Eq. (17). $\lambda$ denotes the preload, in fact, the linear guide rail can be preloaded directly by increasing the roller diameter. Therefore, if the roller diameter is increased by $\lambda$, the actual deformation is expressed as Eq. (16).

$$
\begin{gathered}
\Delta=\left(L_{i j k x}{ }^{2}+L_{i j k y}{ }^{2}\right)^{1 / 2}-D_{a}+\lambda \\
L_{i j k}{ }^{2}=L_{i j k x}{ }^{2}+L_{i j k y}{ }^{2}
\end{gathered}
$$

The center distance component of raceway after deformation in $x$ and $y$ direction is written as Eq. (18). Besides, the angle between the center line of raceway and horizontal direction after deformation is also as follows.

$$
\left\{\begin{array}{l}
L_{i j k x}=L_{i j k} \times \cos \alpha^{\prime} \\
L_{i j k y}=L_{i j k} \times \sin \alpha^{\prime} \\
\alpha^{\prime}=\alpha+\theta
\end{array}\right.
$$

The kinematic error of linear guide rail is expressed as the pose error of slider and guide rail, the pose error of slider and guide rail at a certain position is resulted from the comprehensive factors, namely the geometric error and the elastic deformation under external load. Relative to ideal position, there occurs five groups of errors for slider and guide rail, namely the horizontal displacement error $\delta_{x}, u_{z}$ along $x$ direction, the vertical displacement error $\delta_{y}, v_{T}$ along $y$ direction, the angular error pitch $\varepsilon_{x}, \alpha_{T}$ around $x$ axis, 
the angular error yaw $\varepsilon_{y}, \beta_{z}$ around $y$ axis, and the angular error of roll $\varepsilon_{z}, \delta_{T}$ around $z$ axis. Thus, take the left guide rail as an example. Due to the smaller error value, according to the theory of different motion of robots [28], the error equivalence model of a single slider is established, namely the geometric error of assembly surface of linear guide rail and the pose error of slider is equivalent to the elastic deformation of roller. The pose error transformation matrix of the slider and guide rail can be expressed as Eq. (19) and Eq. (20), respectively.

$$
\begin{aligned}
\Delta T_{s} & =\left[\begin{array}{cccc}
1 & -\varepsilon_{z} & \varepsilon_{y} & \delta_{x} \\
\varepsilon_{z} & 1 & -\varepsilon_{x} & \delta_{y} \\
-\varepsilon_{y} & \varepsilon_{x} & 1 & 0 \\
0 & 0 & 0 & 1
\end{array}\right] \\
\Delta T_{g} & =\left(\begin{array}{cccc}
1 & \delta_{T} & 0 & u_{z} \\
\delta_{T} & 1 & -\alpha_{T} & V_{T} \\
\beta_{z} & \alpha_{T} & 1 & 0 \\
0 & 0 & 0 & 1
\end{array}\right)
\end{aligned}
$$

Taking a single-row four-track guide rail as an example, assuming that the vector of the curvature center of the raceway is Eq. (21), corresponding to the jth roller in the ith raceway. Therefore, the deviations of the pose errors of slider and guide rail in the horizontal and vertical directions are respectively written as Eq. (22) and Eq. (23).

$$
\begin{gathered}
\left(\begin{array}{ll}
x_{i j k} \quad y_{i j k} \quad z_{i j k} & 1
\end{array}\right)^{T} \\
\left\{\begin{array}{l}
\mu_{i j k x}=\delta_{x}+\varepsilon_{y} \cdot z_{i j k}-\varepsilon_{z} \cdot y_{i j k} \\
\mu_{i j k y}=\delta_{y}-\varepsilon_{x} \cdot z_{i j k}+\varepsilon_{z} \cdot x_{i j k}
\end{array}\right. \\
\left\{\begin{array}{l}
\delta_{i j k x}=u_{z}-\delta_{T} \cdot y_{i j k}+\beta_{z} \cdot z_{i j k} \\
\delta_{i j k y}=V_{T}+\delta_{T} \cdot x_{i j k}-\alpha_{T} \cdot z_{i j k}
\end{array}\right.
\end{gathered}
$$

Then, caused by the geometric error and the elastic deformation, the horizontal displacement $L_{i j k x}$ and vertical displacement $L_{i j k y}$ of the center distance of raceway are written as

$$
\left\{\begin{array}{l}
L_{i j k x}=L_{x 0}-\mu_{i j k x}+\delta_{i j k x} \\
L_{i j k y}=L_{y o}-\mu_{i j k y}+\delta_{i j k y}
\end{array}\right.
$$

Finally, according to Eqs. (9)-(24), the kinematic error of a single slider is obtained, its 
formula is expressed as

$$
\left\{\begin{array}{l}
\delta_{x}=L_{x 0}-L_{i j k x}+u_{z} \\
\delta_{y}=L_{y o}-L_{i j k y}+V_{T} \\
\varepsilon_{x}=\alpha_{T} \\
\varepsilon_{y}=\beta_{z} \\
\varepsilon_{z}=\delta_{T}
\end{array}\right.
$$

\subsection{Kinematic error model of the linear axis}

The geometric error of base and the kinematic error of a single slider are studied in above sections in this paper, then the kinematic error of the linear axis is the main research object in this section. In addition, the kinematic error of the linear axis is the same as that of a single slider, and there are five categories of errors: straightness along $x$ and $y$ axis, pitch, yaw, and roll angular error. In this section, the kinematic error of a single slider is transformed to derive and obtain the kinematic error of the linear axis. As shown in Fig. 12, the linear axis of machine tool is composed of base, linear guide rail and carriage.

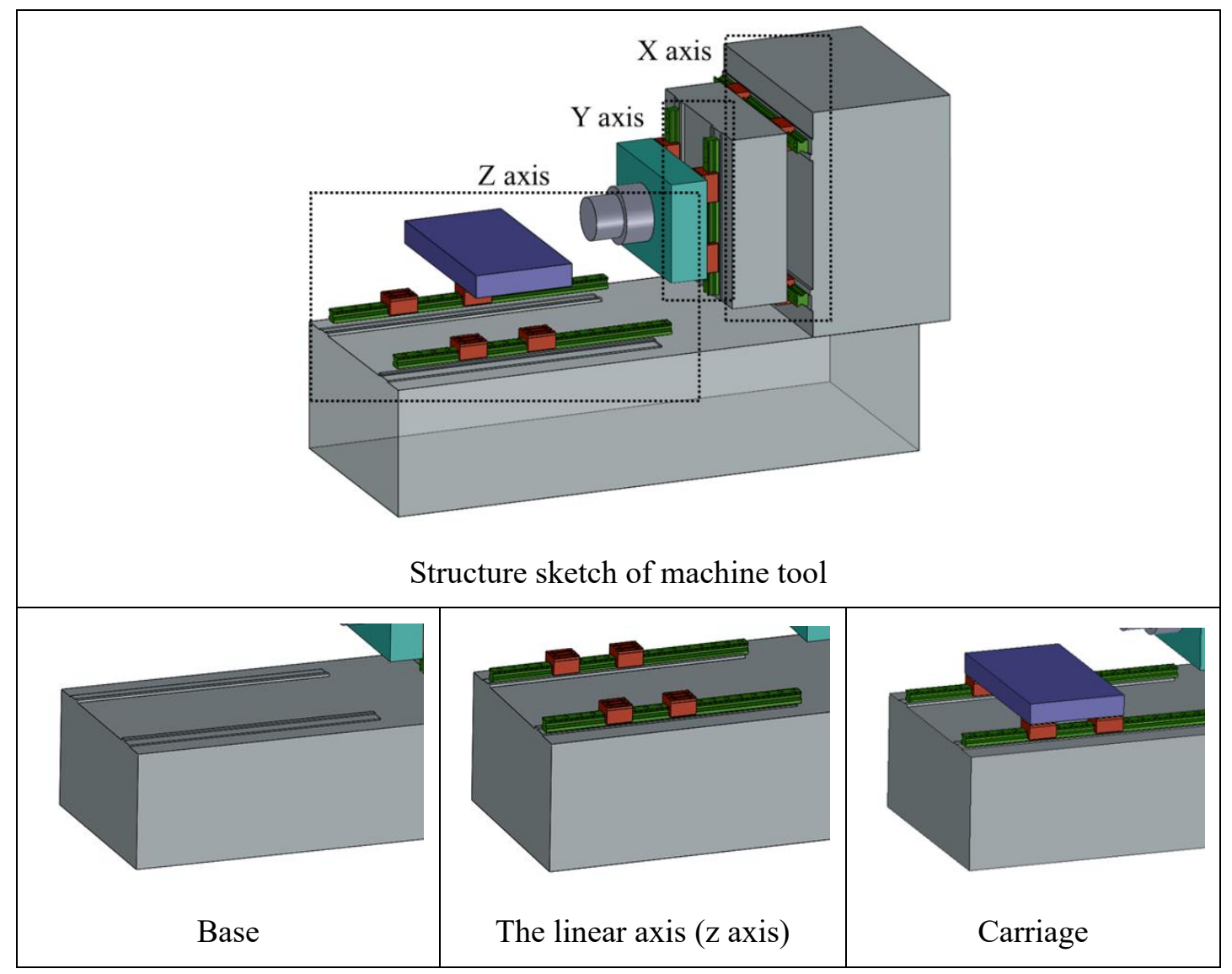




\begin{tabular}{|c|c|c|}
\hline Geometric error & $\begin{array}{c}\text { Kinematic errors of } \\
\text { a single slider }\end{array}$ & $\begin{array}{c}\text { Kinematic errors of } \\
\text { the linear axis }\end{array}$ \\
\hline
\end{tabular}

Fig. 12 All level errors of the linear axis in machine tool

As for force analysis of each slider of the linear guide rail, when the workpiece is milled in machine tool, apart from the weight of carriage and workpiece, the most important load is the milling force. The total load of slider keeps balanced in the horizontal and vertical direction, besides the gravity and milling force are decomposed into horizontal and vertical components. Therefore, the horizontal load and vertical load of each slider are calculated as in Eq. (26)-(27).

$$
\begin{gathered}
\left\{\begin{array}{l}
F_{V 1}=P_{1}+P_{1}^{\prime} \\
F_{V 2}=P_{2}+P_{2}^{\prime} \\
F_{V 3}=P_{3}+P_{3}^{\prime} \\
F_{V 4}=P_{4}+P_{4}^{\prime}
\end{array}\right. \\
\left\{\begin{array}{l}
F_{H 1}=P_{1 T} \\
F_{H 2}=P_{2 T} \\
F_{H 3}=P_{3 T} \\
F_{H 4}=P_{4 T}
\end{array}\right.
\end{gathered}
$$

where $P_{i}$ represents the vertical load of each slider under the workpiece gravity $G$ in Fig. 13, similarly, $P_{i}^{\prime}$ is the vertical load of each slider under the milling force $F_{m}, P_{i T}$ denotes the horizontal load of each slider under the milling force $F_{m}$ in Fig. 14, $d_{s}$ is the distance between two sliders along $z$ axis, $b_{g}$ is the distance between two guide rails along $x$ axis, $l_{1}$ is the distance from the gravity center to the carriage center along $z$ axis, $l_{2}$ is the distance from the gravity center to the carriage center along $x$ axis, $l_{3}$ is the distance from the milling force point to the carriage center along $z$ axis, $l_{4}$ is the distance from the milling force point to the slider center along $y$ axis. 


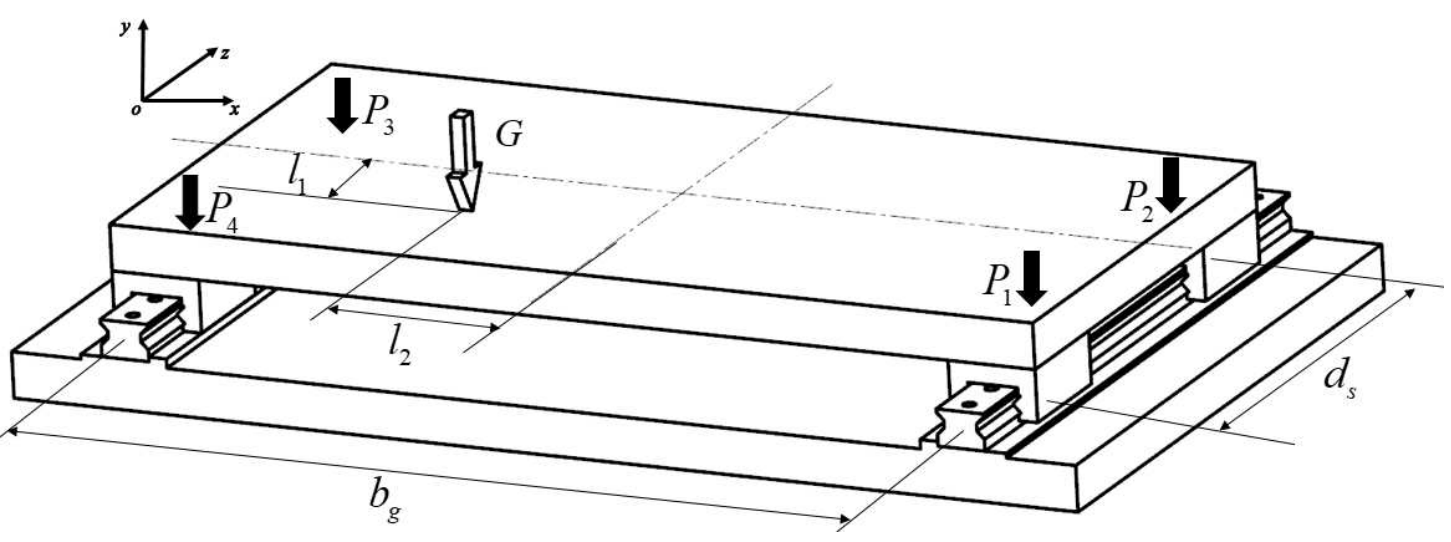

Fig. 13 Force analysis of each slider under the workpiece gravity

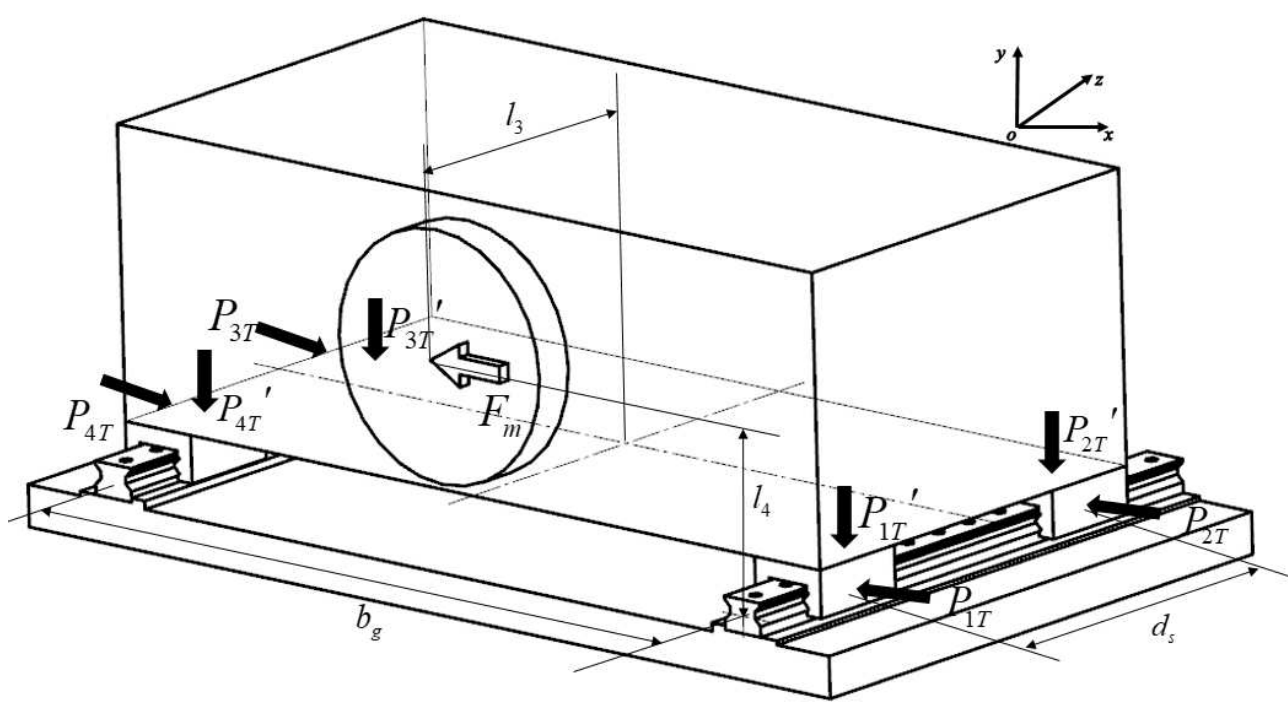

Fig. 14 Force analysis of each slider under the milling force

The gravity component of each slider is calculated as Eq. (28), and the milling force of each slider in horizontal and vertical direction is calculated as Eq. (29).

$$
\left\{\begin{array}{l}
P_{1}=\frac{G}{4}+\frac{G \cdot l_{1}}{2 \cdot d_{s}}-\frac{G \cdot l_{2}}{2 \cdot b_{g}} \\
P_{2}=\frac{G}{4}-\frac{G \cdot l_{1}}{2 \cdot d_{s}}-\frac{G \cdot l_{2}}{2 \cdot b_{g}} \\
P_{3}=\frac{G}{4}-\frac{G \cdot l_{1}}{2 \cdot d_{s}}+\frac{G \cdot l_{2}}{2 \cdot b_{g}} \\
P_{4}=\frac{G}{4}+\frac{G \cdot l_{1}}{2 \cdot d_{s}}+\frac{G \cdot l_{2}}{2 \cdot b_{g}}
\end{array}\right.
$$




$$
\left\{\begin{array}{l}
P_{1}^{\prime}=P_{2}^{\prime}=-\frac{F_{m} \cdot l_{4}}{2 \cdot b_{g}} \\
P_{3}^{\prime}=P_{4}^{\prime}=\frac{F_{m} \cdot l_{4}}{2 \cdot b_{g}} \\
P_{1 T}=P_{4 T}=-\frac{F_{m}}{4}-\frac{F_{m} \cdot l_{3}}{2 \cdot d_{s}} \\
P_{2 T}=P_{3 T}=-\frac{F_{m}}{4}+\frac{F_{m} \cdot l_{3}}{2 \cdot d_{s}}
\end{array}\right.
$$

By substituting Eqs. (28)-(29) into (26)-(27), the horizontal and vertical load of each slider can be calculated. Afterwards, Eqs. (26)-(27) are substituted into Eq. (12) in section 3.1. Finally, through the derivation and calculation of formulas in section 3.1, the kinematic error of each slider under the workpiece gravity and milling force can be obtained.

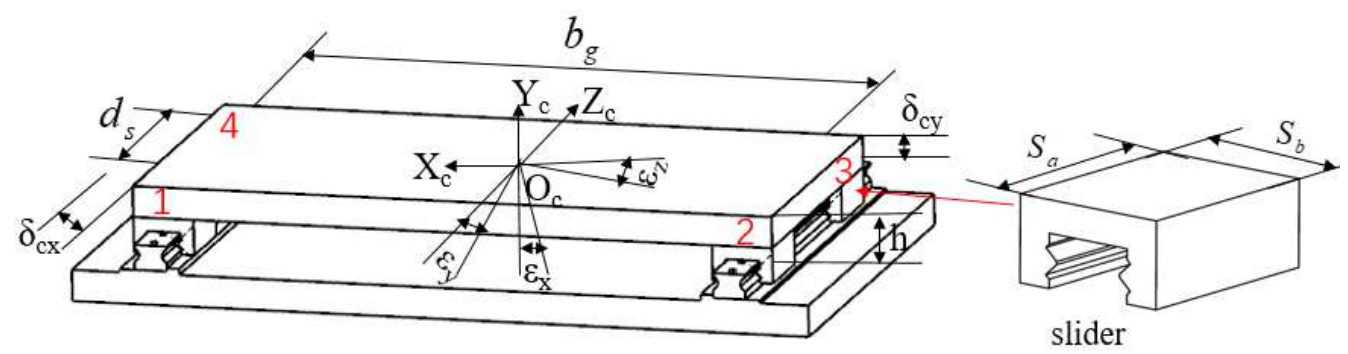

Fig. 15 Pose error derivation of carriage

As for the straightness solution of carriage in horizontal and vertical direction, based on the principle of vector summation, the straightness of each slider in horizontal and vertical direction are mapped into the carriage [29], aiming to calculate the kinematic error of the linear axis. So the carriage error parameters are deduced in Eqs. (30)-(33), number 1-4 of the following Eqs. (30)-(33) stands for the carriage position corresponding to slider position in Fig. 15.

$$
\begin{aligned}
& \left\{\begin{array}{l}
\Delta_{1 x}=\delta_{1 x}-d_{s} / 2 \cdot \sin \varepsilon_{1 y}+h \cdot \sin \varepsilon_{1 z} \\
\Delta_{1 y}=\delta_{1 y}-d_{s} / 2 \cdot \sin \varepsilon_{1 x}-b_{g} / 2 \cdot \sin \varepsilon_{1 z}
\end{array}\right. \\
& \left\{\begin{array}{l}
\Delta_{2 x}=\delta_{2 x}+d_{s} / 2 \cdot \sin \varepsilon_{2 y}+h \cdot \sin \varepsilon_{2 z} \\
\Delta_{2 y}=\delta_{2 y}+d_{s} / 2 \cdot \sin \varepsilon_{2 x}-b_{g} / 2 \cdot \sin \varepsilon_{2 z}
\end{array}\right. \\
& \left\{\begin{array}{l}
\Delta_{3 x}=\delta_{3 x}+d_{s} / 2 \cdot \sin \varepsilon_{3 y}+h \cdot \sin \varepsilon_{3 z} \\
\Delta_{3 y}=\delta_{3 y}+d_{s} / 2 \cdot \sin \varepsilon_{3 x}+b_{g} / 2 \cdot \sin \varepsilon_{3 z}
\end{array}\right.
\end{aligned}
$$




$$
\left\{\begin{array}{l}
\Delta_{4 x}=\delta_{4 x}-d_{s} / 2 \cdot \sin \varepsilon_{4 y}+h \cdot \sin \varepsilon_{4 z} \\
\Delta_{4 y}=\delta_{4 y}-d_{s} / 2 \cdot \sin \varepsilon_{4 x}+b_{g} / 2 \cdot \sin \varepsilon_{4 z}
\end{array}\right.
$$

where $\Delta_{i x}$ is the straightness of carriage position corresponding to slider position in horizontal direction, the same to the $\Delta_{i y}$ in vertical direction. $\delta_{i x}$ denotes the straightness of each slider in horizontal direction, $\delta_{i y}$ is the straightness of each slider in vertical direction, $\varepsilon_{i x}$ is the pitch angular error of each slider around $x$ axis, $\varepsilon_{i y}$ is the yaw angular error of each slider around $y$ axis, $\varepsilon_{i z}$ is the roll angular error of each slider around $z$ axis and the variables mentioned above, $i=1,2,3$, 4. In Fig. 15, $h$ denotes the distance from the top surface of carriage to a slider center along $y$ axis.

Through a series of derivations and calculations, the straightness of the linear axis in horizontal and vertical direction are expressed as

$$
\left\{\begin{array}{l}
\delta_{c x}=-\Delta_{1 x}-\Delta_{2 x}+\Delta_{3 x}+\Delta_{4 x} \\
\delta_{c y}=\Delta_{1 y}+\Delta_{2 y}-\Delta_{3 y}-\Delta_{4 y}
\end{array}\right.
$$

As for the angular errors solution (pitch, yaw and roll) of carriage, the angular error of carriage is determined by itself straightness and manufacturing error. Therefore, the angular error calculation (pitch, yaw and roll) of four positions of carriage corresponding to slider positions is shown in Eqs. (35)-(38) below.

$$
\begin{aligned}
& \left\{\begin{array}{l}
\mathrm{E}_{3 x}=\delta_{3 y}+\varepsilon_{3 x} \cdot\left(S_{a} / 2\right) \\
\mathrm{E}_{3 y}=\delta_{3 x}+\varepsilon_{3 y} \cdot\left(S_{a} / 2\right) \\
\mathrm{E}_{3 z}=\delta_{3 y}+\varepsilon_{3 z} \cdot\left(S_{b} / 2\right)
\end{array}\right. \\
& \left\{\begin{array}{l}
\mathrm{E}_{2 x}=\delta_{2 y}+\varepsilon_{2 x} \cdot\left(S_{a} / 2\right) \\
\mathrm{E}_{2 y}=\delta_{2 x}+\varepsilon_{2 y} \cdot\left(S_{a} / 2\right) \\
\mathrm{E}_{2 z}=\delta_{2 y}+\varepsilon_{2 z} \cdot\left(S_{b} / 2\right)
\end{array}\right.
\end{aligned}
$$




$$
\begin{array}{r}
\left\{\begin{array}{l}
\mathrm{E}_{4 x}=\delta_{4 y}+\varepsilon_{4 x} \cdot\left(S_{a} / 2\right) \\
\mathrm{E}_{4 y}=\delta_{4 x}+\varepsilon_{4 y} \cdot\left(S_{a} / 2\right) \\
\mathrm{E}_{4 z}=\delta_{4 y}+\varepsilon_{4 z} \cdot\left(S_{b} / 2\right)
\end{array}\right. \\
\left\{\begin{array}{l}
\mathrm{E}_{1 x}=\delta_{1 y}+\varepsilon_{1 x} \cdot\left(S_{a} / 2\right) \\
\mathrm{E}_{1 y}=\delta_{1 x}+\varepsilon_{1 y} \cdot\left(S_{a} / 2\right) \\
\mathrm{E}_{1 z}=\delta_{1 y}+\varepsilon_{1 z} \cdot\left(S_{b} / 2\right)
\end{array}\right.
\end{array}
$$

where $E_{i x}(i=1,2,3,4)$ is the pitch error of carriage position corresponding to slider position around $x$ axis, the same to the $E_{i y}$ and $E_{i z}(i=1,2,3,4)$ around $y$ and $z$ axis. In Fig. 15, $S_{a}$ is the length of slider, $S_{b}$ is the width of slider, $S_{c}$ is the height of slider.

Through a series of derivations and calculations, the angular error (pitch, yaw and roll) of the linear axis around $x, y$ and $z$ are expressed as

$$
\left\{\begin{array}{l}
\varepsilon_{c x}=\arctan \left(\left|\left(\mathrm{E}_{4 x}-\mathrm{E}_{1 x}\right) / 2-\left(\mathrm{E}_{2 x}-\mathrm{E}_{3 x}\right) / 2\right| / d_{s}\right) \\
\varepsilon_{c y}=\arctan \left(\left|\left(\mathrm{E}_{4 y}-\mathrm{E}_{1 y}\right) / 2-\left(\mathrm{E}_{2 y}-\mathrm{E}_{3 y}\right) / 2\right| / d_{s}\right) \\
\varepsilon_{c z}=\arctan \left(\left|\left(\mathrm{E}_{4 x}+\mathrm{E}_{3 x}\right) / 2-\left(\mathrm{E}_{1 x}+\mathrm{E}_{2 x}\right) / 2\right| / b_{g}\right)
\end{array}\right.
$$

Hence, by adopting the error equivalent model, the kinematic error of the linear axis is obtained, as shown in Eq. (40).

$$
\left\{\begin{array}{l}
\delta_{c x}=-\Delta_{1 x}-\Delta_{2 x}+\Delta_{3 x}+\Delta_{4 x} \\
\delta_{c y}=\Delta_{1 y}+\Delta_{2 y}-\Delta_{3 y}-\Delta_{4 y} \\
\varepsilon_{c x}=\arctan \left(\left|\left(\mathrm{E}_{4 x}-\mathrm{E}_{1 x}\right) / 2-\left(\mathrm{E}_{2 x}-\mathrm{E}_{3 x}\right) / 2\right| / d_{s}\right) \\
\varepsilon_{c y}=\arctan \left(\left|\left(\mathrm{E}_{4 y}-\mathrm{E}_{1 y}\right) / 2-\left(\mathrm{E}_{2 y}-\mathrm{E}_{3 y}\right) / 2\right| / d_{s}\right) \\
\varepsilon_{c z}=\arctan \left(\left|\left(\mathrm{E}_{4 x}+\mathrm{E}_{3 x}\right) / 2-\left(\mathrm{E}_{1 x}+\mathrm{E}_{2 x}\right) / 2\right| / b_{g}\right)
\end{array}\right.
$$

\section{Experimental validation of kinematic error model}

In order to verify the accuracy of the proposed model in this paper, a 4-axis horizontal machining center of a company is taken as a case. The $z$-axis geometric error of the machine tool was measured, including the straightness in horizontal and vertical direction and angular 
deviation of pitch, yaw and roll. Five measurement results of geometric error are compared with the corresponding calculation results of the proposed theoretical model, aiming to verify the proposed model.

\subsection{Experiment setup}

Some approaches have been utilizied to measure the kinematic error of the linear axis [30], in this paper, the horizontal machine tool of a certain company is regarded as a case to carry out the verification experiments. The Renishaw multi-laser interferometer XL-80 is employed to measure angular errors ( pitch and yaw) of $z$-axis of machine tool, the instrument resolution is $0.01^{\prime \prime}$, the angle measurement range is $\pm 10^{\circ}$, the angle accuracy is $( \pm 0.2 \% \pm$ $0.5 \pm 0.1 \mathrm{M}) \mu \mathrm{m} / \mathrm{m}, \mathrm{M}$ is the measurement distance, the unit is $\mathrm{m}$. The dial indicator and marble square are adopted to measure the straightness along the plane $x o z$ and $y o z$, the electronic level meter is used to measure the angular error (roll), as shown in Fig. 16. Besides , the type NSK RA55 of the linear guide rail is used in the machien tool, some parameters is listed in Table 2. All guide rail, roller and slider of linear guide rail use the material GCr15, the elasticity modulus is $206 \mathrm{GPa}$, and the Poisson's ratio is 0.3 . Therfore, all five kinematic errors of z-axis of horizontal machine tool have been measured [31], as shown in Fig. 17. 


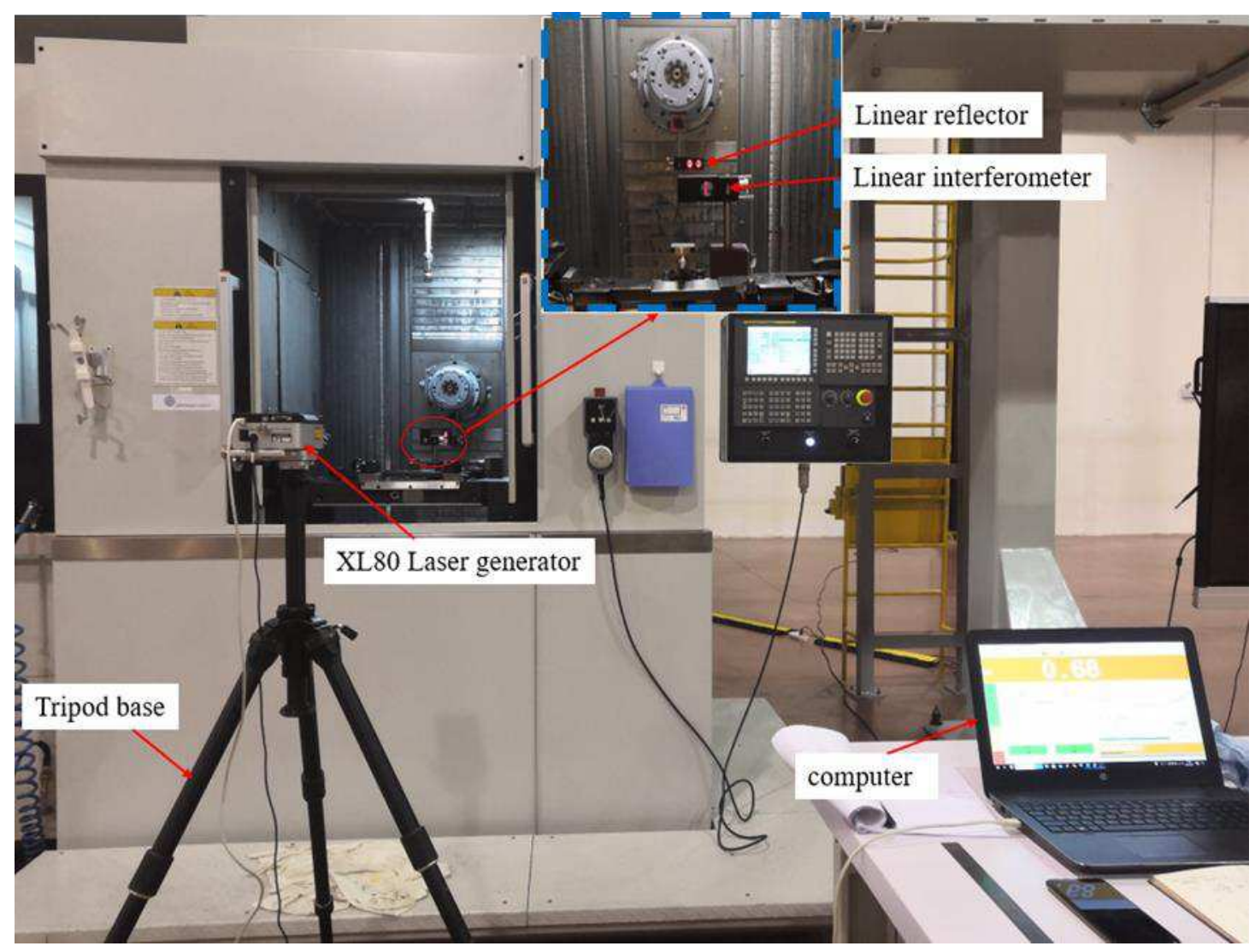

Fig. 16 Renishaw equipment of multi-laser interferometer

Table 2 Parameters of NSK guide rail RA55

\begin{tabular}{ll}
\hline & Type \\
Items & RA55 \\
\hline
\end{tabular}

Slider dimension /mm

$128 \times 98 \times 70$

Guide rail dimension $/ \mathrm{mm}$

$1316 \times 53 \times 43.5$

Roller diameter $/ \mathrm{mm}$

6

Roller contact length /mm

Static bearing capacity /N

Dynamic bearing capacity / $\mathrm{N}$

129000

Moderate preload /N

4361

Initial contact angle $/^{\circ}$

Number of grooves 
After the dial indicator and marble square are adjusted, and the linear axis of machine tool is moved along $z$ axis, then the straightness is measured. The top and side surface of the marble square is measured with the dial indicator, respectively, namely the straightness of surface yoz and $x o z$ along z axis in Fig. 17 (a) and (b). Secondly, after the laser interferometer is adjusted, and the linear axis of machine tool is also moved along $\mathrm{z}$ axis, the angular error of pitch and yaw are measured in Fig. 17 (c) and (d). Finally, after the electronic level is adjusted, and the linear axis of machine tool is also moved along $z$ axis, the angular error of roll is measured in Fig. 17 (e). In addition, the $z$ axis moving stroke of the machine tool is $610 \mathrm{~mm}$. The above moving along the $\mathrm{z}$ axis of machine tool are set up 10 measuring points each stroke and repeated for 10 times. A total of 100 groups of measurement data are obtained.

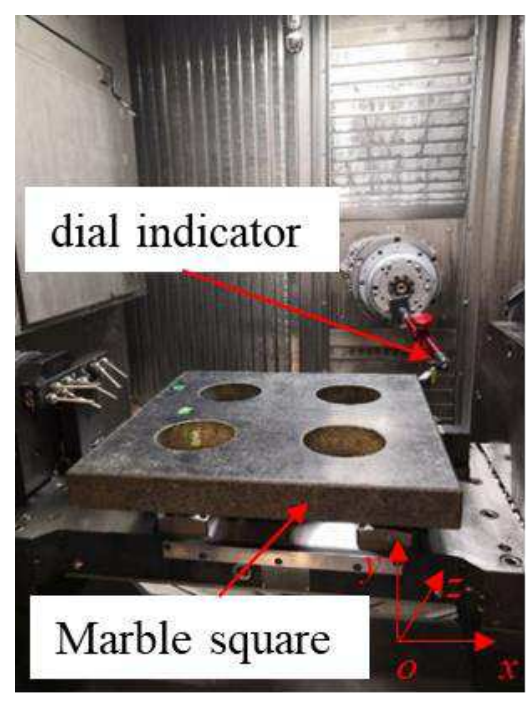

(a)

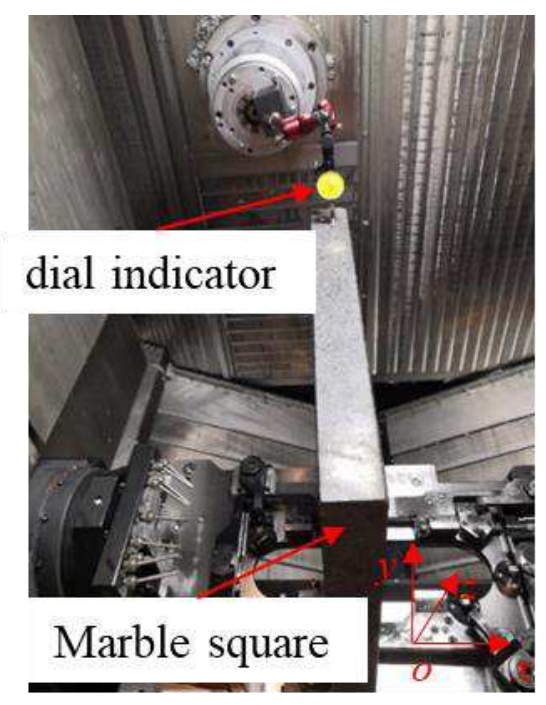

(b) 


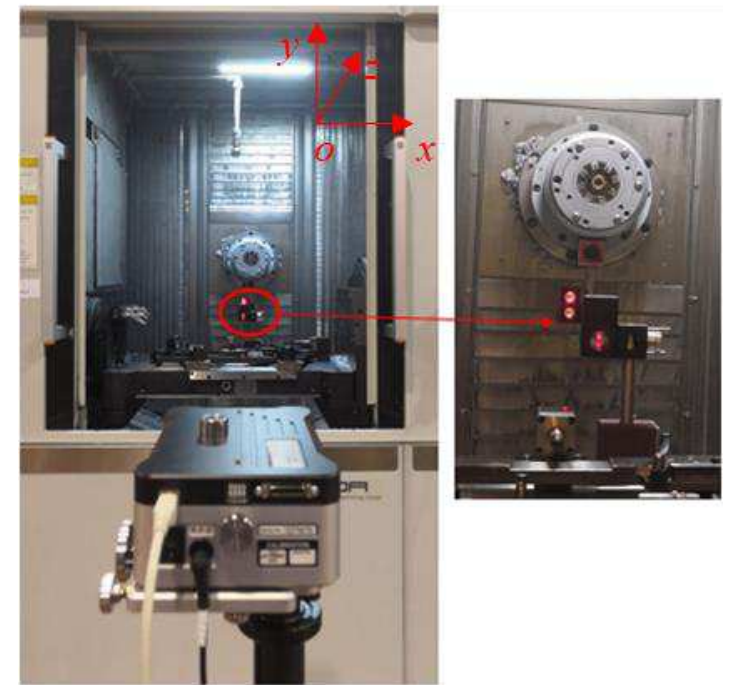

(c)

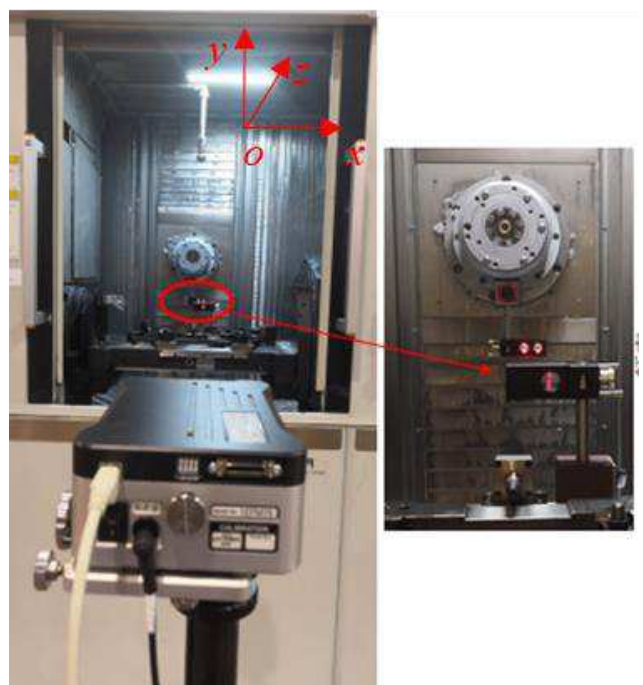

(d)

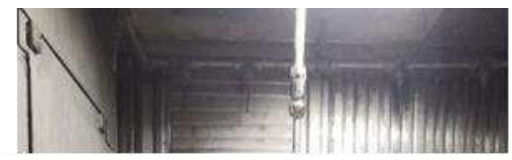

\section{Electronic level meter}

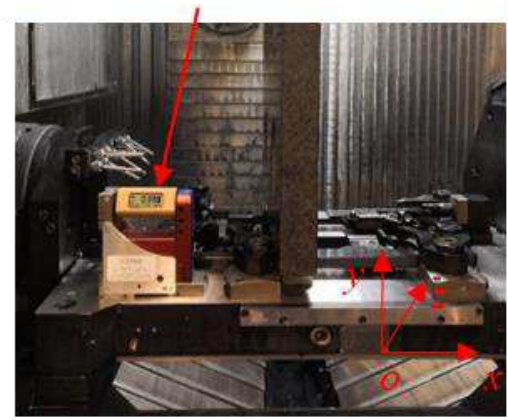

(e)

Fig. 17 Measurement of kinematic error of $z$-axis of horizontal machine tool

(a) Straightness of the yoz plane; (b) Straightness of the xoz plane; (c) yoz plane angular error around $x$-axis (pitch); (d) xoz plane angular error around $y$-axis (yaw); (e) xoy plane angular error around the $z$

$$
\text { axis (roll) }
$$

\subsection{Verification}

With measured straightness and angular error of $z$ axis of machine tool, the corresponding kinematic errors of the linear axis are all calculated by the proposed model in this paper, as shown in Fig. 18. In order to verify the validity of the proposed model, the 
theoretical model results are compared with the experiment data, as shown in Fig. 19.

Five groups of measured kinematic error parameters were plotted as the frequency histogram, and then Gaussian fitting was performed to obtain the Gaussian curve. Afterwards, five kinds of kinematic error parameters calculated by the proposed theoretical model were plotted as the frequency vs error curves, moreover the peak lines of the frequency curves of the experiment data were compared and analyzed with the theoretical model. The comparison results show that for five kinds of kinematic errors, the frequency value of the theoretical model curve is much smaller than that measured by the experiment in Table 3 . It is mainly due to the larger sample size of the theoretical model than the experimental model. Furthermore, the comparison mainly focuses on the frequency peak value, namely the average value of kinematic error.

When machine tool moves along $\mathrm{z}$ axis, on one hand, as for the straightness of two surfaces, and surface $x o z$ along $z$ axis, as shown in Table 3, the error of the maximum frequency value between the theoretical model and experiment results is $0.18 \%$ in Fig. 18 (a) and Fig. 19 (a). Similarly, surface $y o z$ along $z$ axis, the error of the maximum frequency value between the theoretical model and experiment results is $0.26 \%$ in Fig. 18 (b) and Fig. 19 (b). On the other hand, as for the angular error around $x, y$ and $z$ axis, the pitch error around $x$ axis between the theoretical model and experiment results is 6.46\% in Fig. 18 (c) and Fig. 19 (c), and the yaw error around $y$ axis between the theoretical model and experiment results is 8.24\% in Fig. 18 (d) and Fig. 19 (d), and the roll error around $z$ axis between the theoretical model and experiment results is $8.54 \%$ in Fig. 18 (e) and Fig. 19 (e).

It can be seen from the above results that the errors of five kinematic error values calculated by the theoretical model are all within $10 \%$, thus it belongs to the acceptable accuracy range. Therefore, the results show that the theoretical model proposed in this paper is accurate and feasible. In addition, the theoretical model proposed in this paper can also provide a scientific and reasonable guidance for the design, manufacture and assembly of the linear axis of the machine tools. 


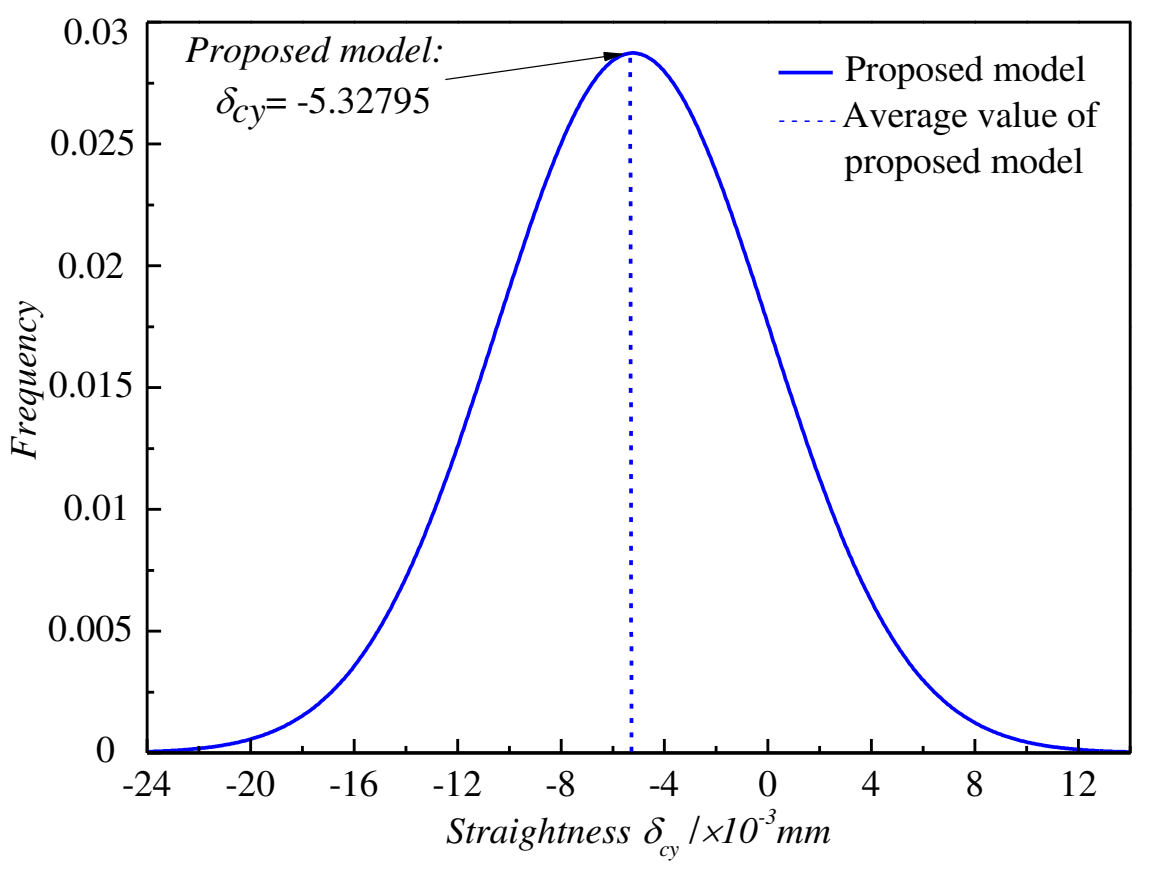

(a)

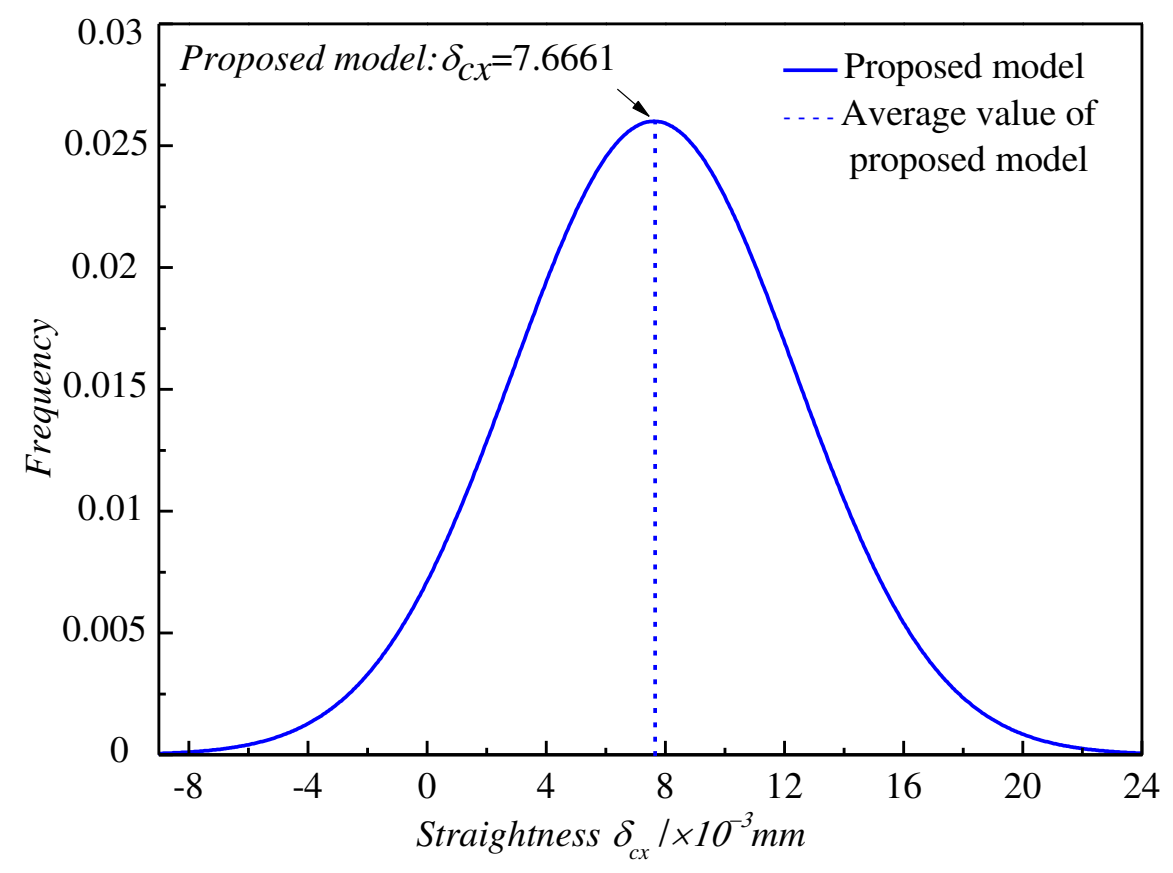

(b) 


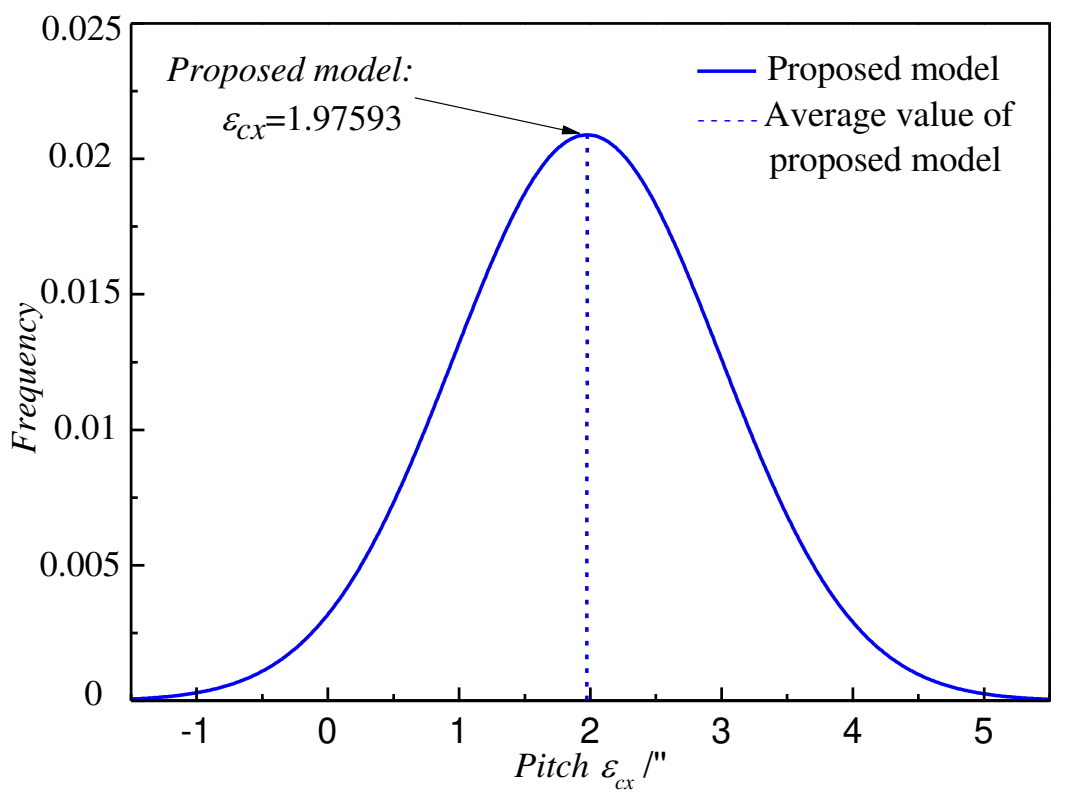

(c)

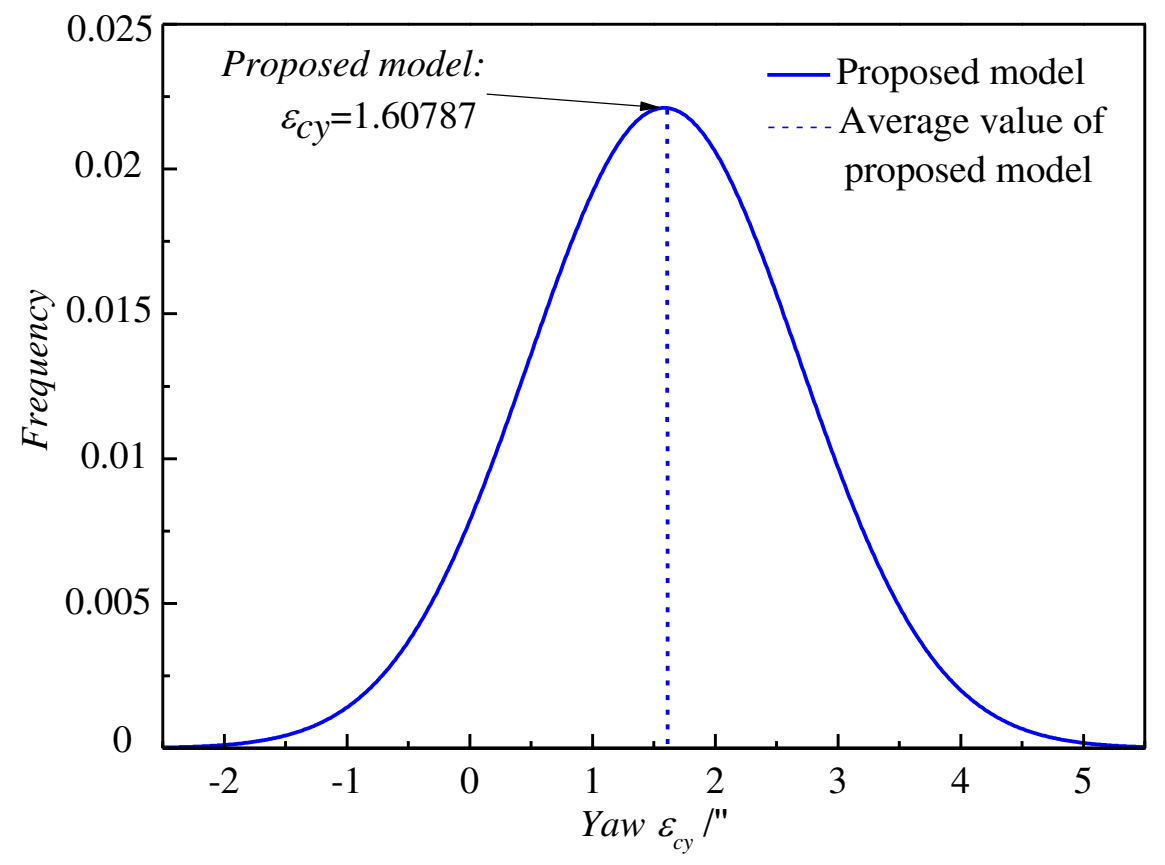

(d) 


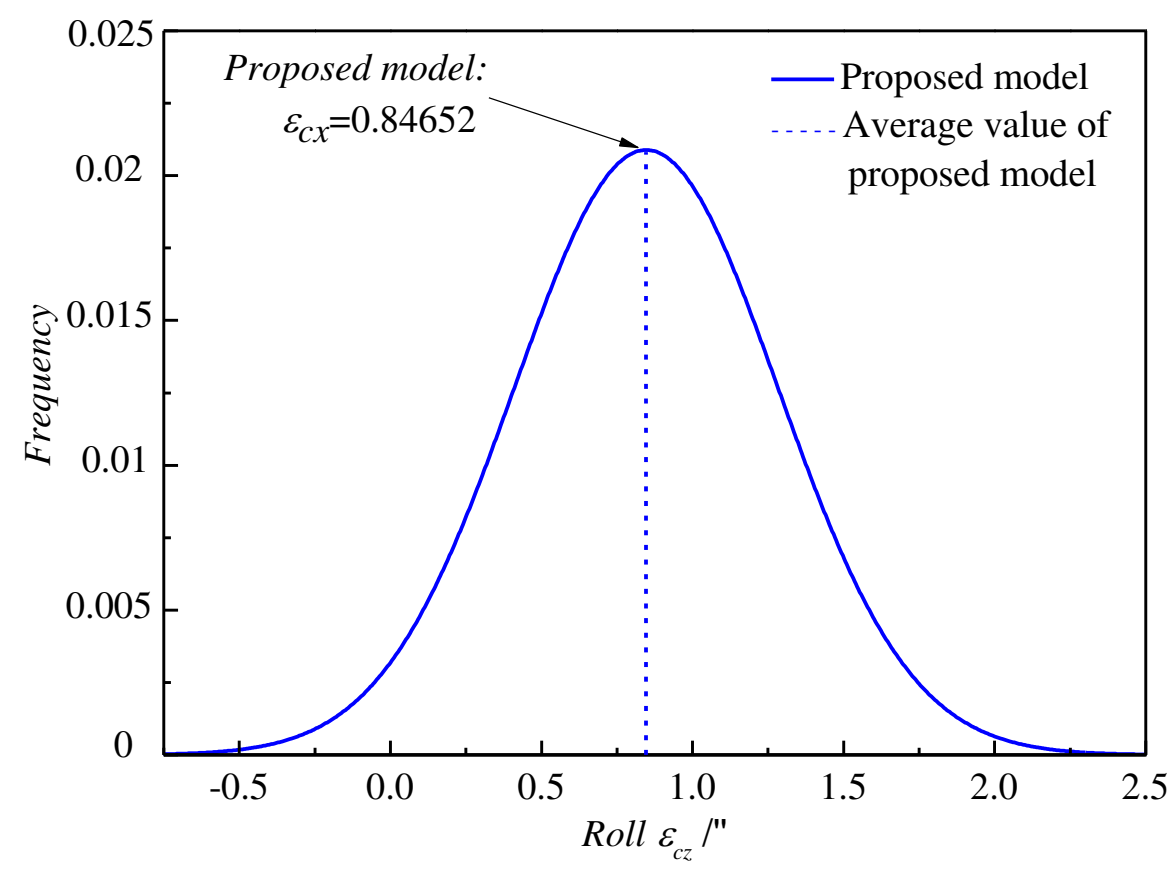

(e)

Fig. 18 Calculated kinematic errors of the linear axis:

(a) Straightness $\delta_{c x}$; (b) Straightness $\delta_{c y}$; (c) Pitch $\varepsilon_{c x}$; (d) Yaw $\varepsilon_{c y}$; (e) Roll $\varepsilon_{c z}$

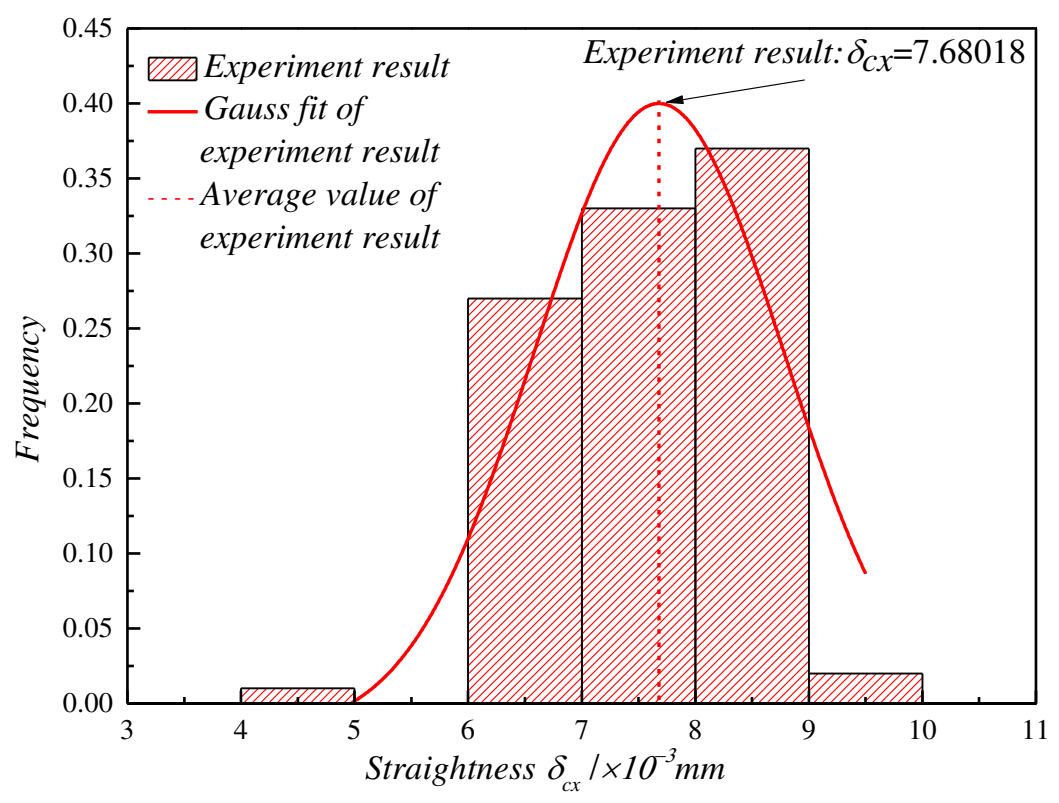

(a) 


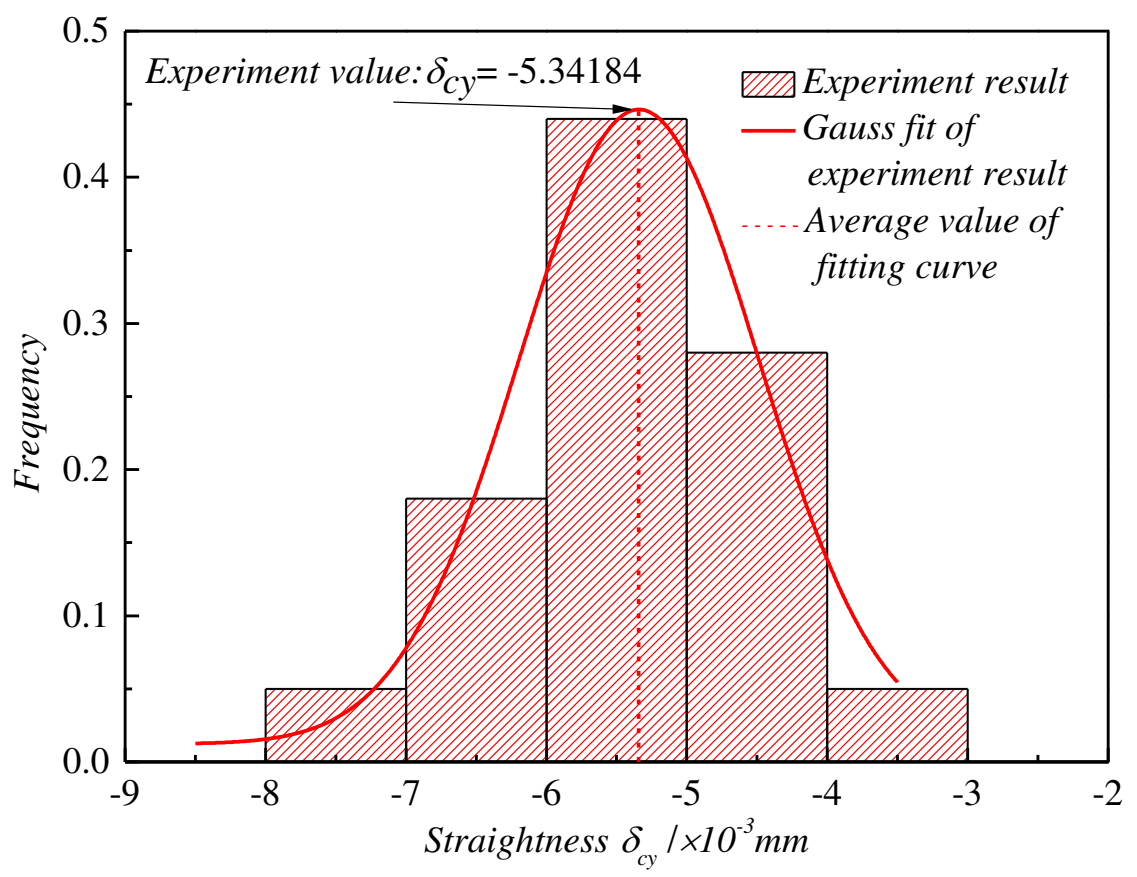

(b)

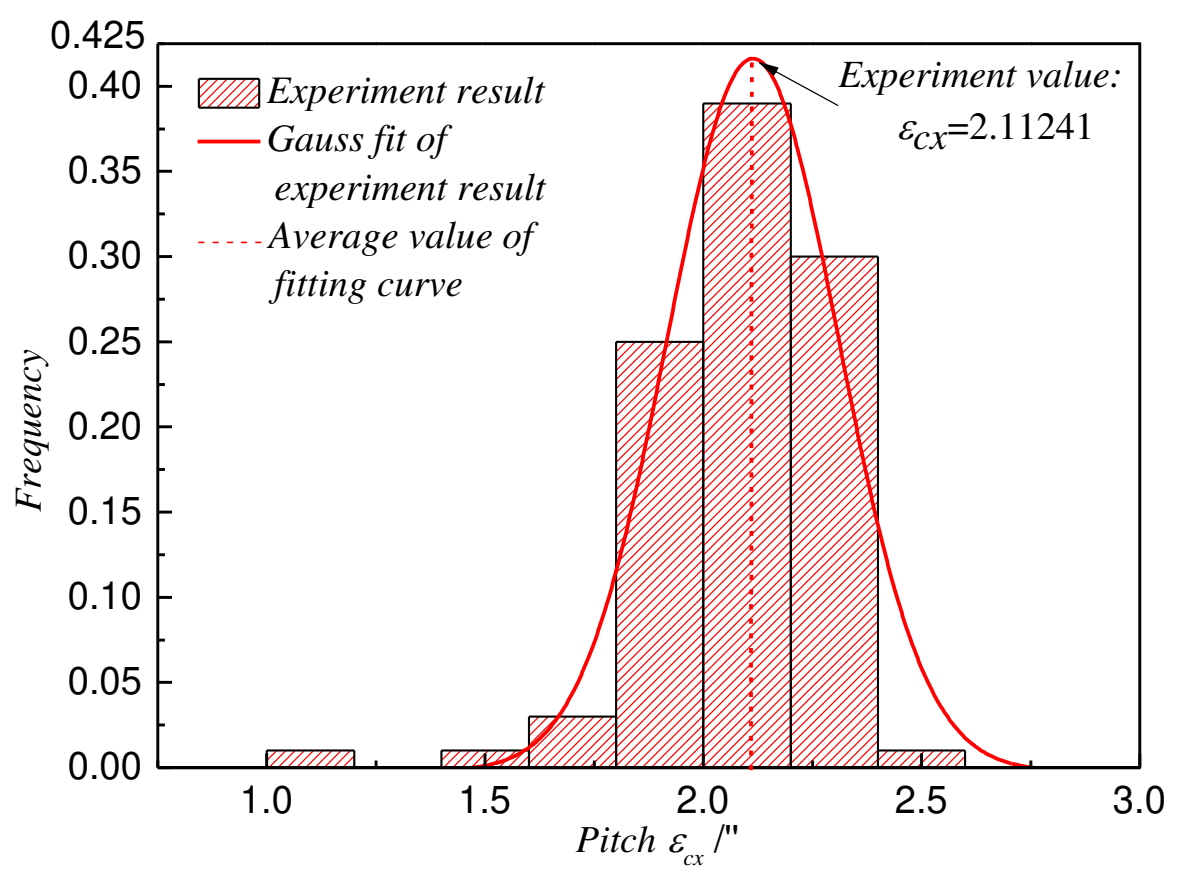

(c) 


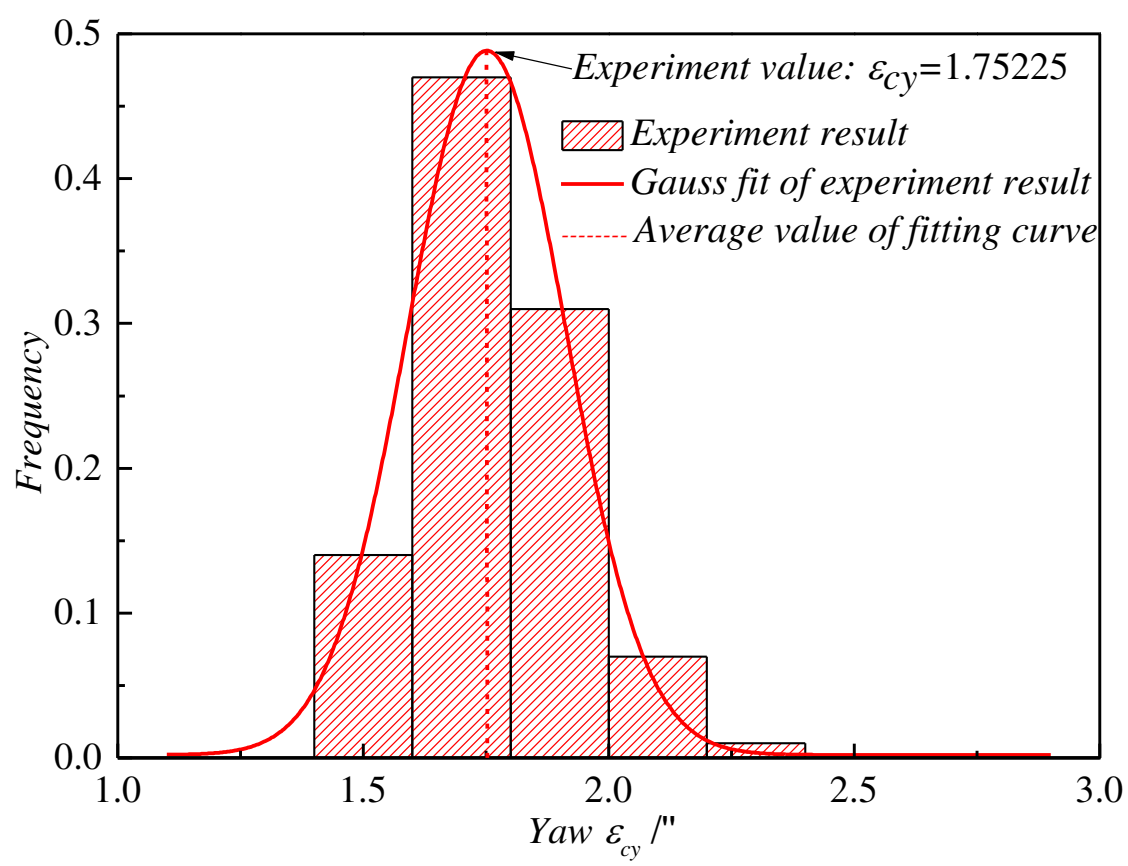

(d)

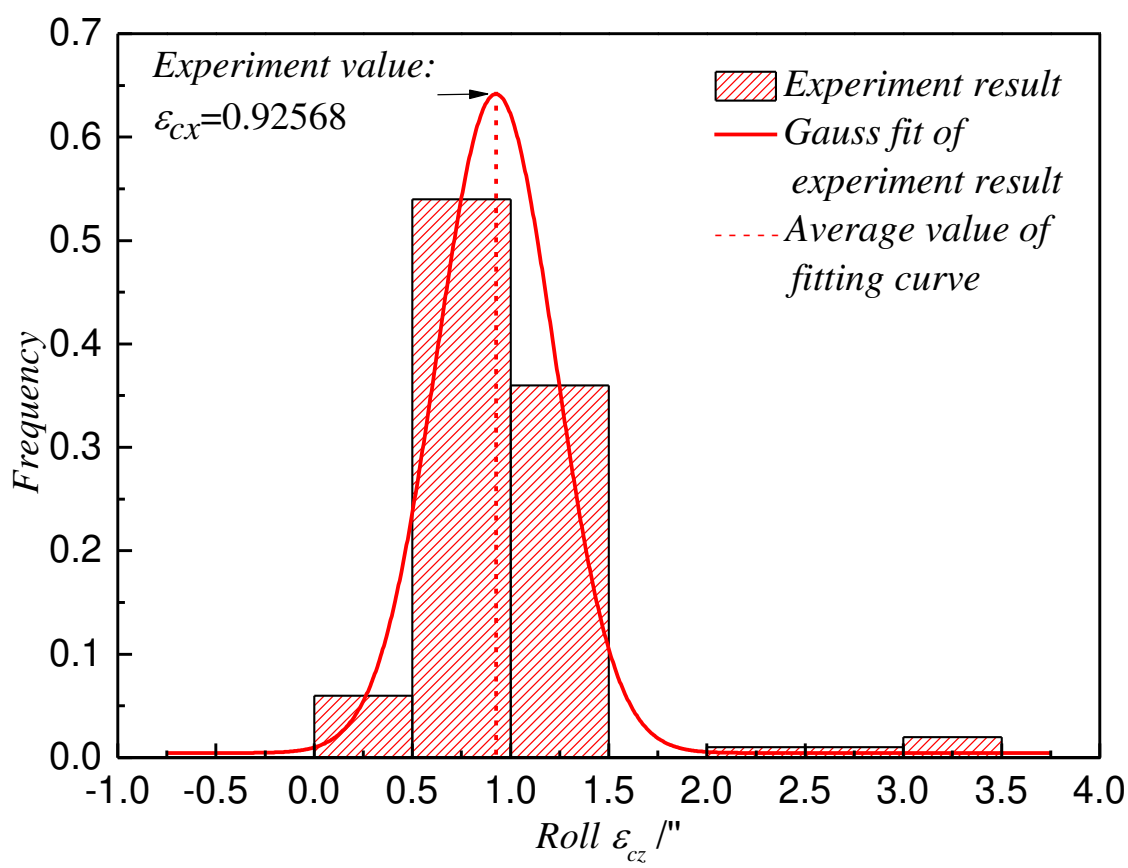

(e)

Fig. 19 Measured kinematic errors of the linear axis:

(a) Straightness $\delta_{c x}$; (b) Straightness $\delta_{c y}$; (c) Pitch $\varepsilon_{c x}$; (d) Yaw $\varepsilon_{c y}$; (e) Roll $\varepsilon_{c z}$ 
Table 3 Errors comparison of the proposed model and experiment measurement for the kinematic

\begin{tabular}{llllll} 
Stroms & Straightness & Straightness & Pitch & Yaw & Roll \\
Experiment result & $\delta_{c x}\left(\times 10^{-3} \mathrm{~mm}\right)$ & $\delta_{c y}\left(\times 10^{-3} \mathrm{~mm}\right)$ & $\varepsilon_{c x}\left({ }^{\prime \prime}\right)$ & $\varepsilon_{c y}\left({ }^{\prime \prime}\right)$ & $\varepsilon_{c z}\left({ }^{\prime \prime}\right)$ \\
Proposed model & 7.68018 & -5.34184 & 2.11241 & 1.75225 & 0.92568 \\
Errors & 7.66611 & -5.32795 & 1.97593 & 1.60787 & 0.84652 \\
\hline
\end{tabular}

\section{Discussions}

In the manufacturing and assembly process of the linear axis, the geometric error of parts and the external load play a key role in making a precision linear axis of the machine tool. According to above verification results in section 4.2, some effect factors on the kinematic error of the linear axis is researched in the proposed model, including the preload of guide rail, the geometric error of the assembly surface of linear guide rail, and the external load and moment.

\subsection{Effect of preload}

According to literatures $[13,32]$, as for a single slider, the preload played an important role in the straightness of the assembly main datum surface $A$ and the subordinate datum surface $B$ or $C$, however it can be seen from Fig. 20 that the preload has almost no influence on all five kinematic errors of the linear axis. It can be inferred that the kinematic errors of four sliders of the linear axis produce the elimination each other. Although the preload has no obvious effect on the kinematic error of the linear axis, the selection of linear guide rail must comply with the machine tool design rules in the other aspects of bearing capacity and accuracy requirements. 


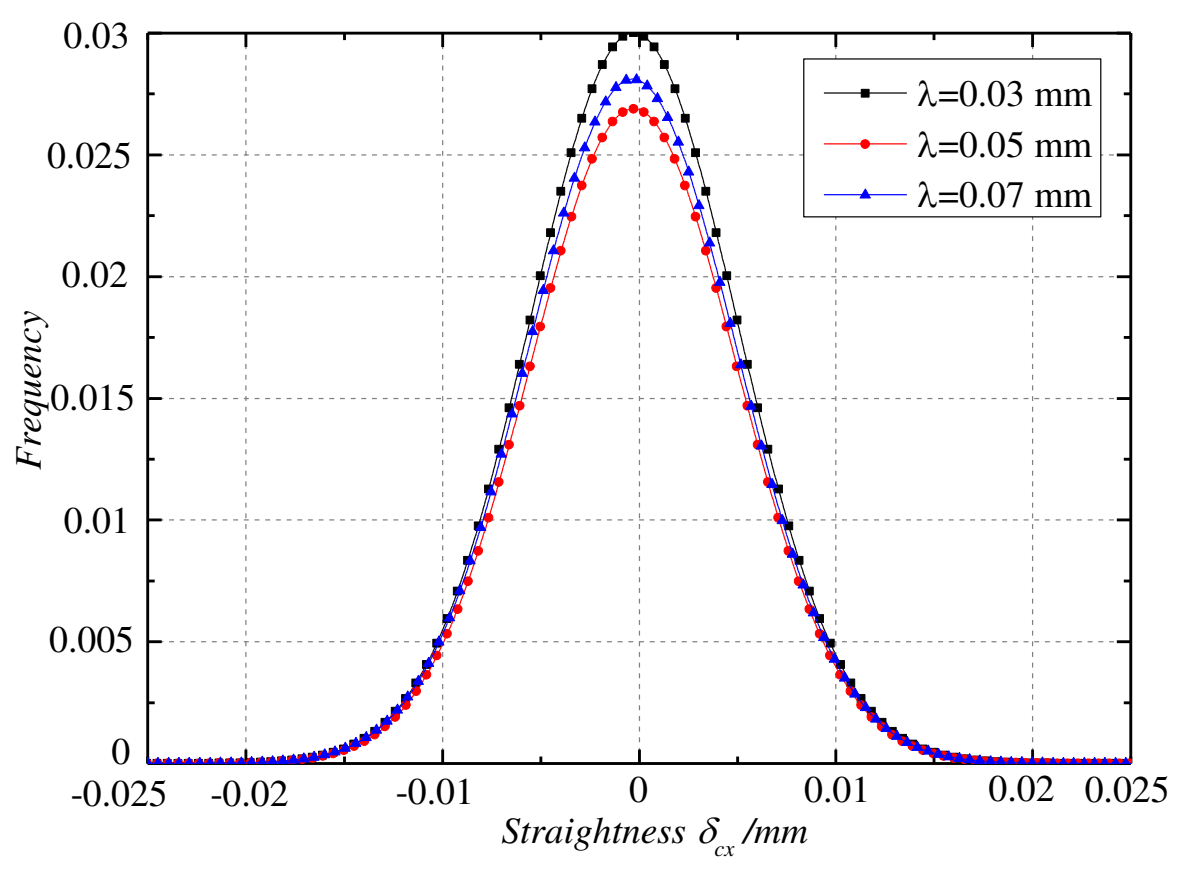

(a)

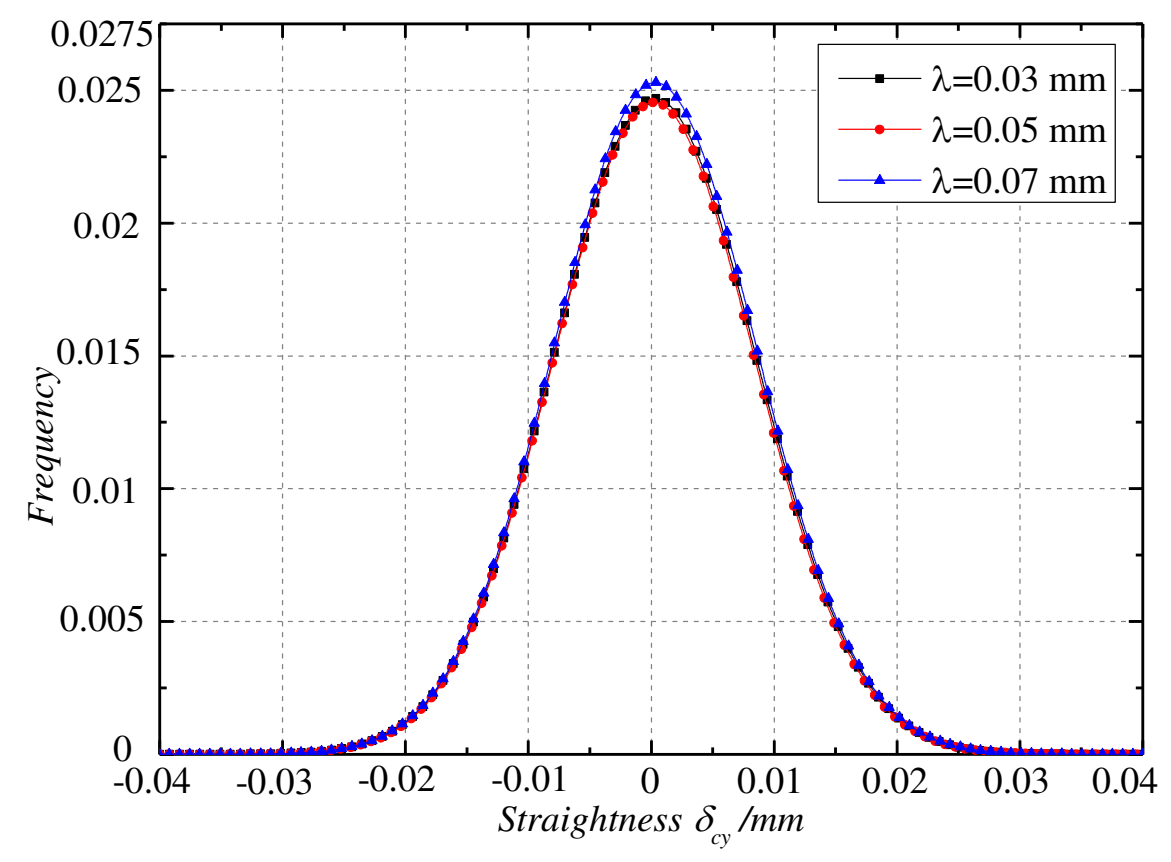

(b) 


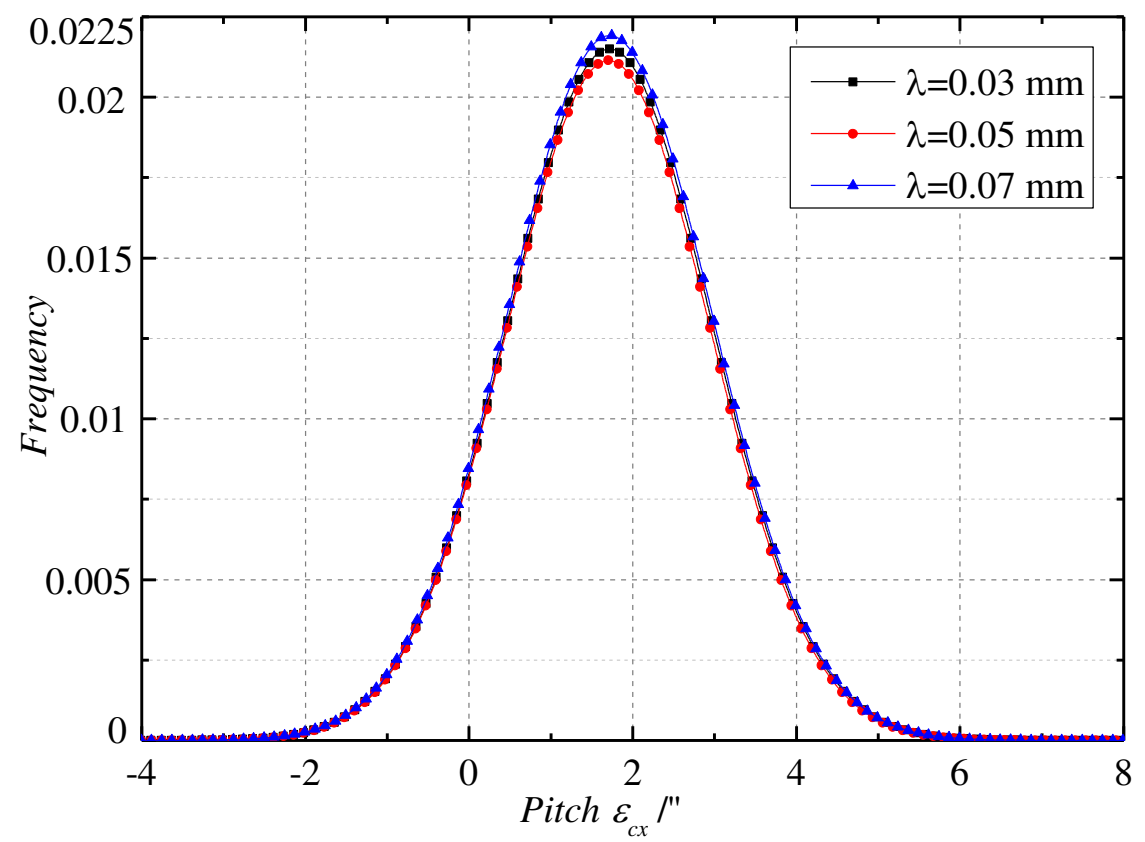

(c)

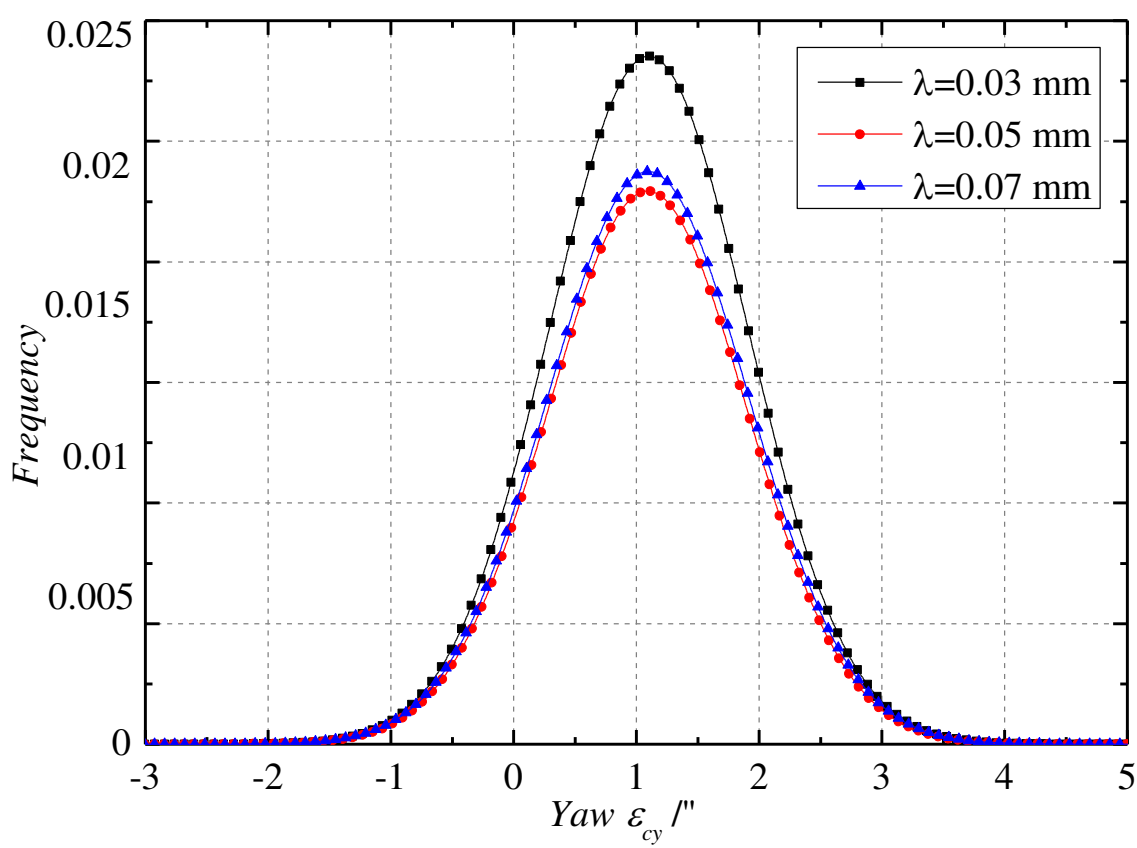

(d) 


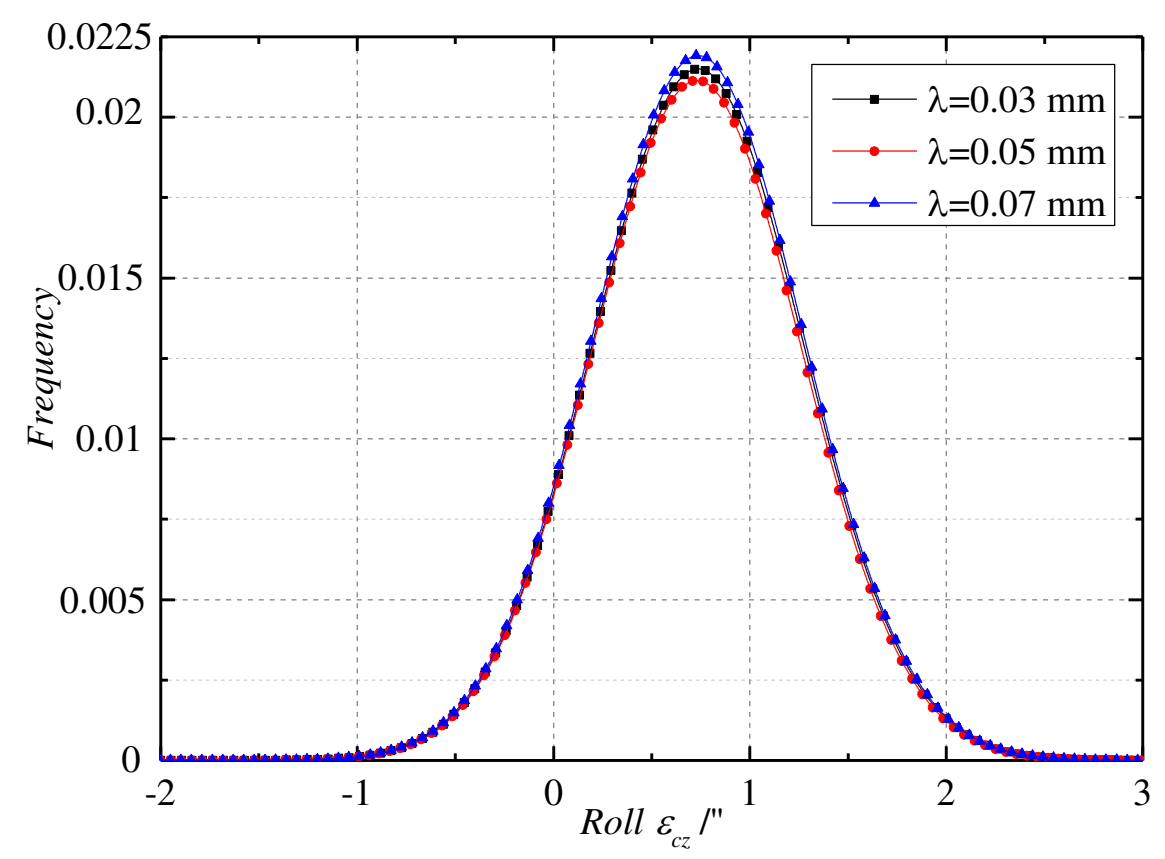

(e)

Fig. 20 Kinematic error of the linear axis under different preload

(a) Straightness $\delta_{c x} ;$ (b) Straightness $\delta_{c y} ;$ (c) Pitch $\varepsilon_{c x}$; (d) Yaw $\varepsilon_{c y}$; (e) Roll $\varepsilon_{c z}$

\subsection{Effect of flatness}

As shown in Fig. 21, the flatness error of the assembly main datum surface $A$ has a significant on the kinematic error of the linear axis, especially the straightness error of surface $x o z$ moving along $z$ axis, and more on the angular error of pitch and roll. With the improvement of precision grade, five kinematic errors of the linear axis show an obvious tendency to decrease, moreover the distribution range shrinks. When the flatness tolerance drops from $0.04 \mathrm{~mm}$ to $0.015 \mathrm{~mm}$, the straightness error of surface $x o z$ of the linear axis decreases from $0.05 \mathrm{~mm}$ to $0.02 \mathrm{~mm}$, and the angular error of pitch decreases from $9 "$ to $3 "$, these results imply remarkable parameter changes. Meanwhile, it is found that the angular error of roll has the same obvious change, and decreases from $3.5 "$ to $1.5 "$. In addition, the proposed model in this paper takes the elastic deformation of rollers into account, hence the mean center line of the kinematic error curve has a deviation and is not at the position of zero 
point.

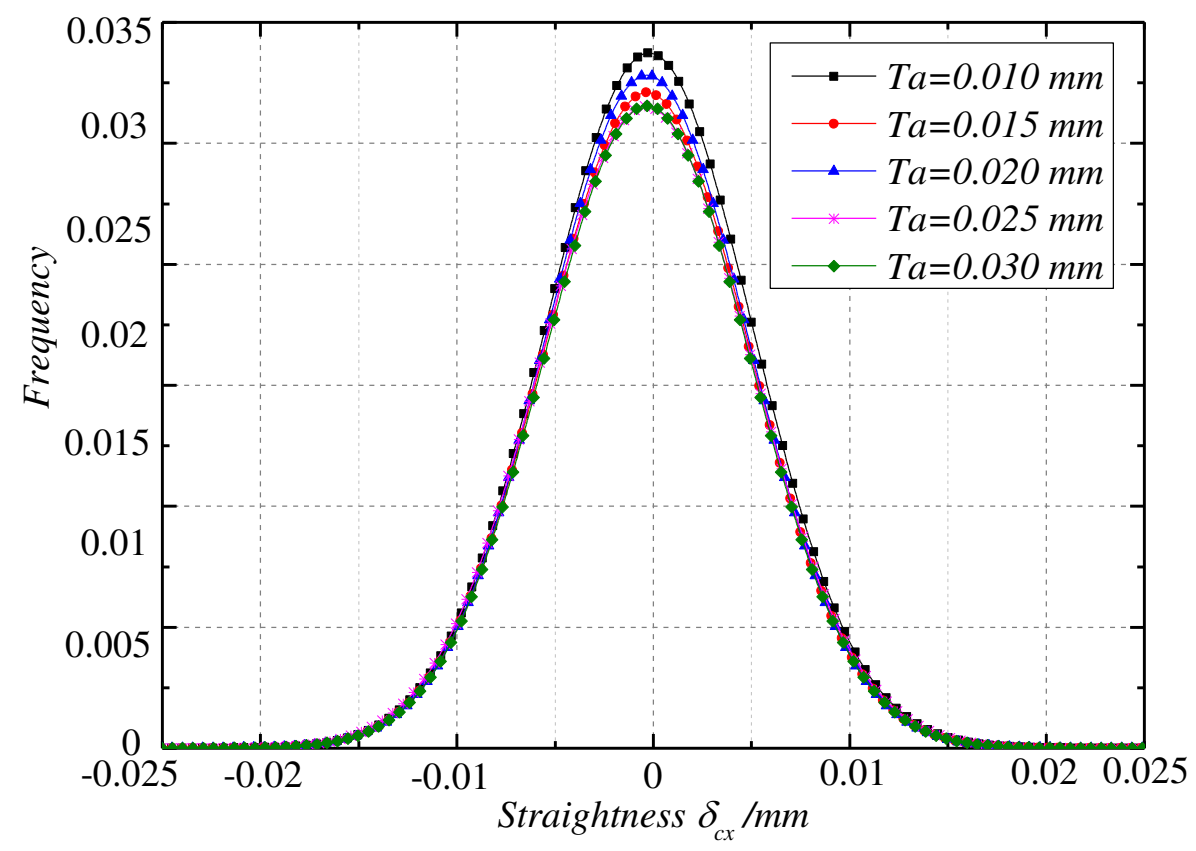

(a)

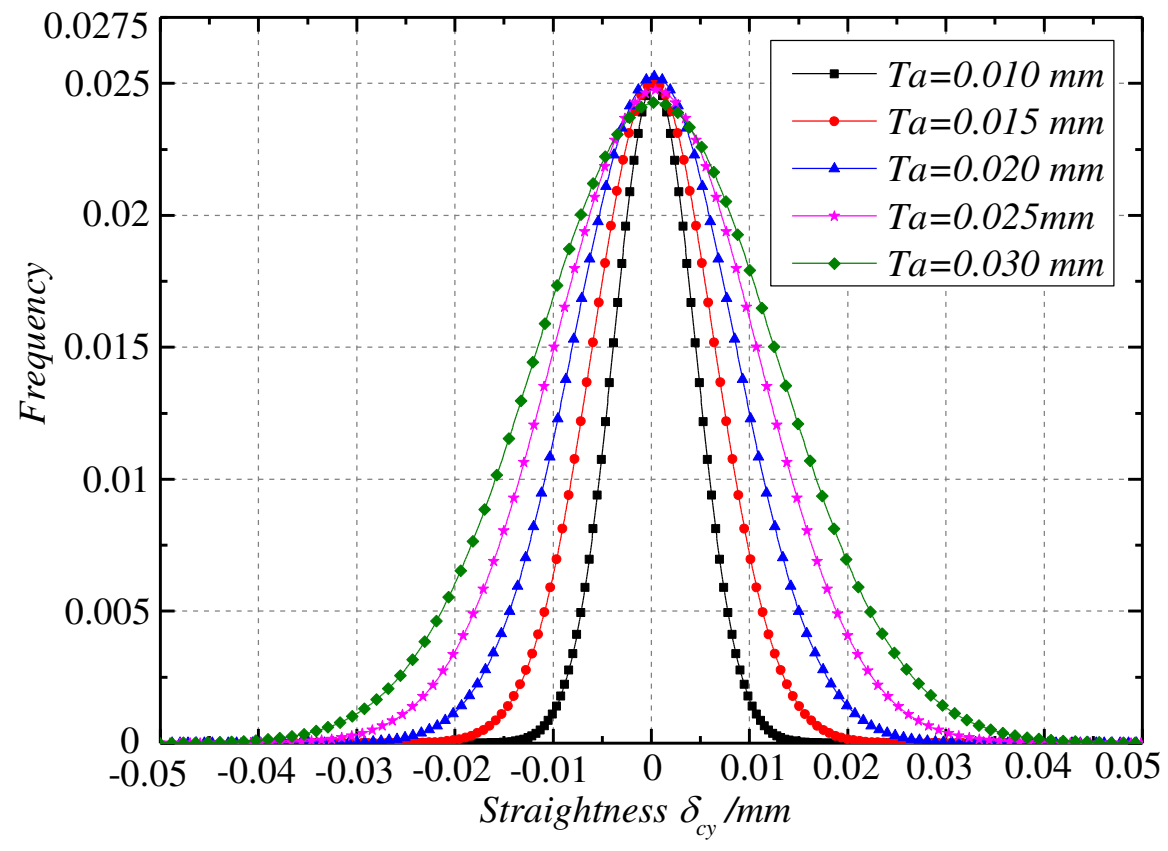

(b) 


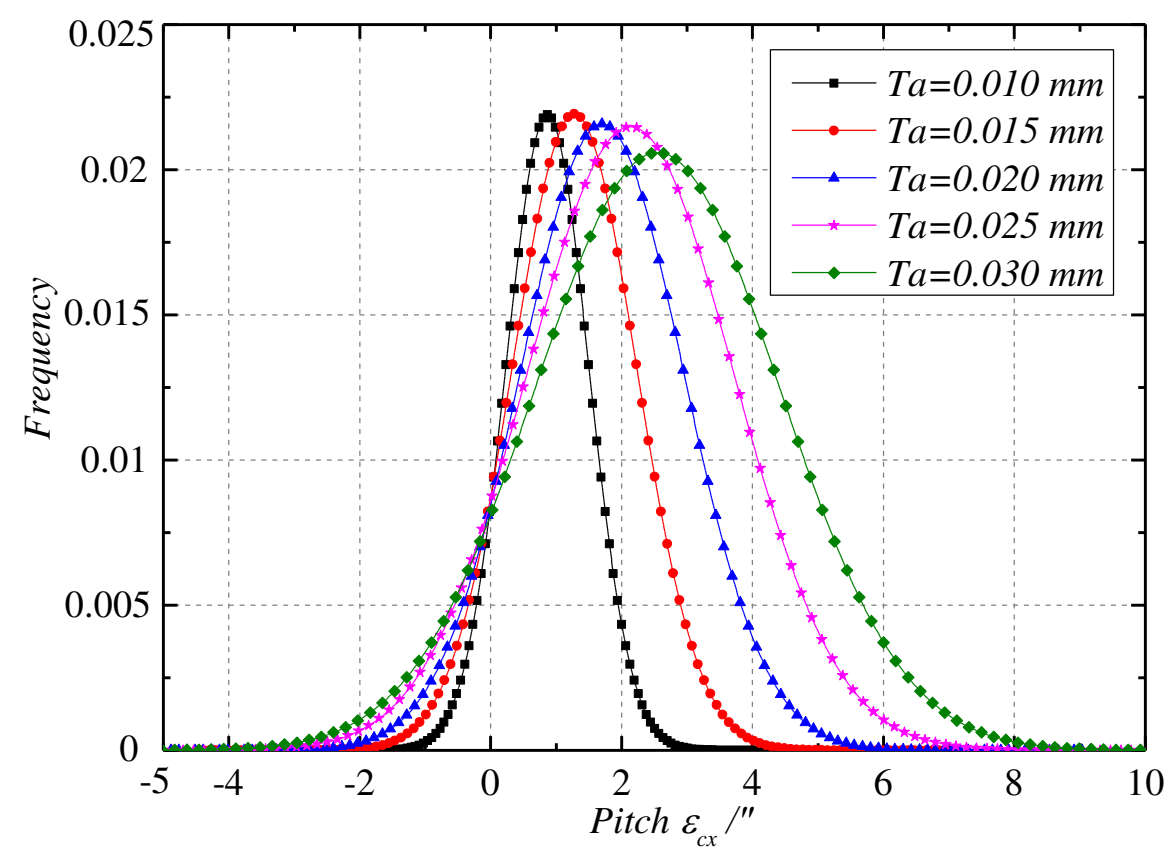

(c)

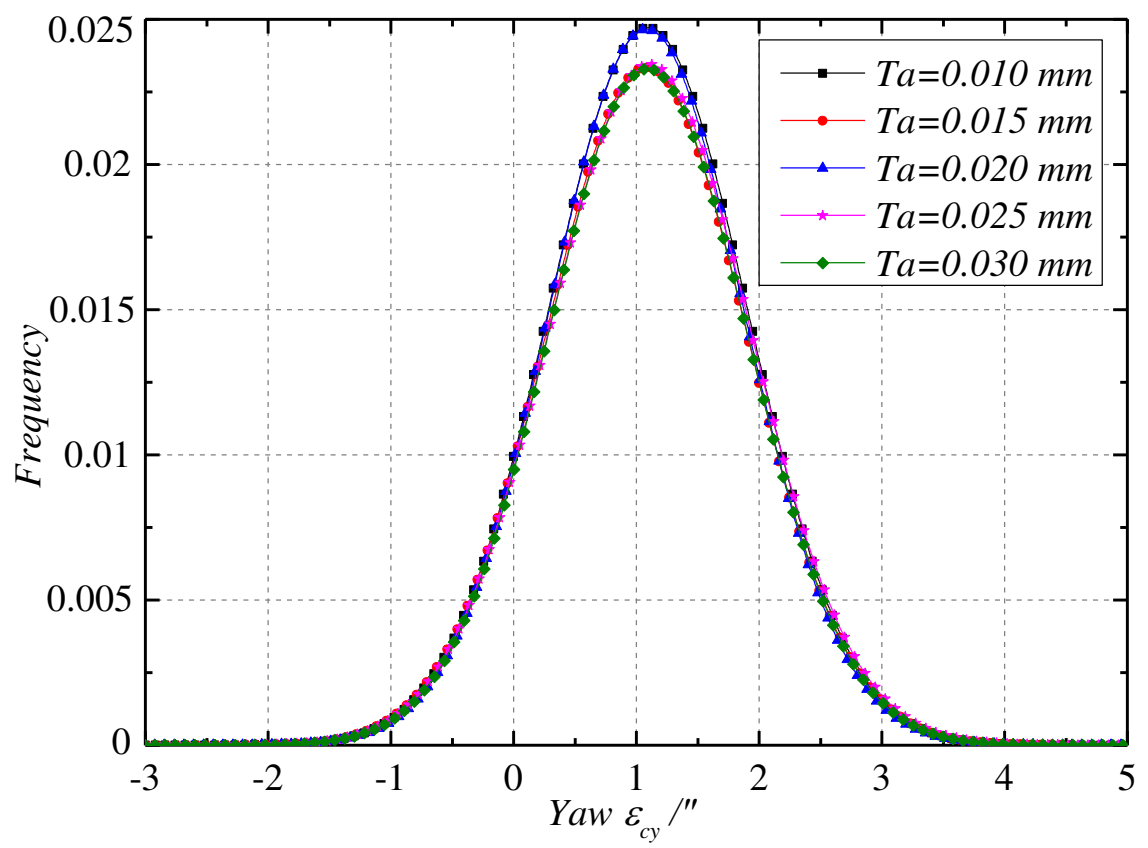

(d) 


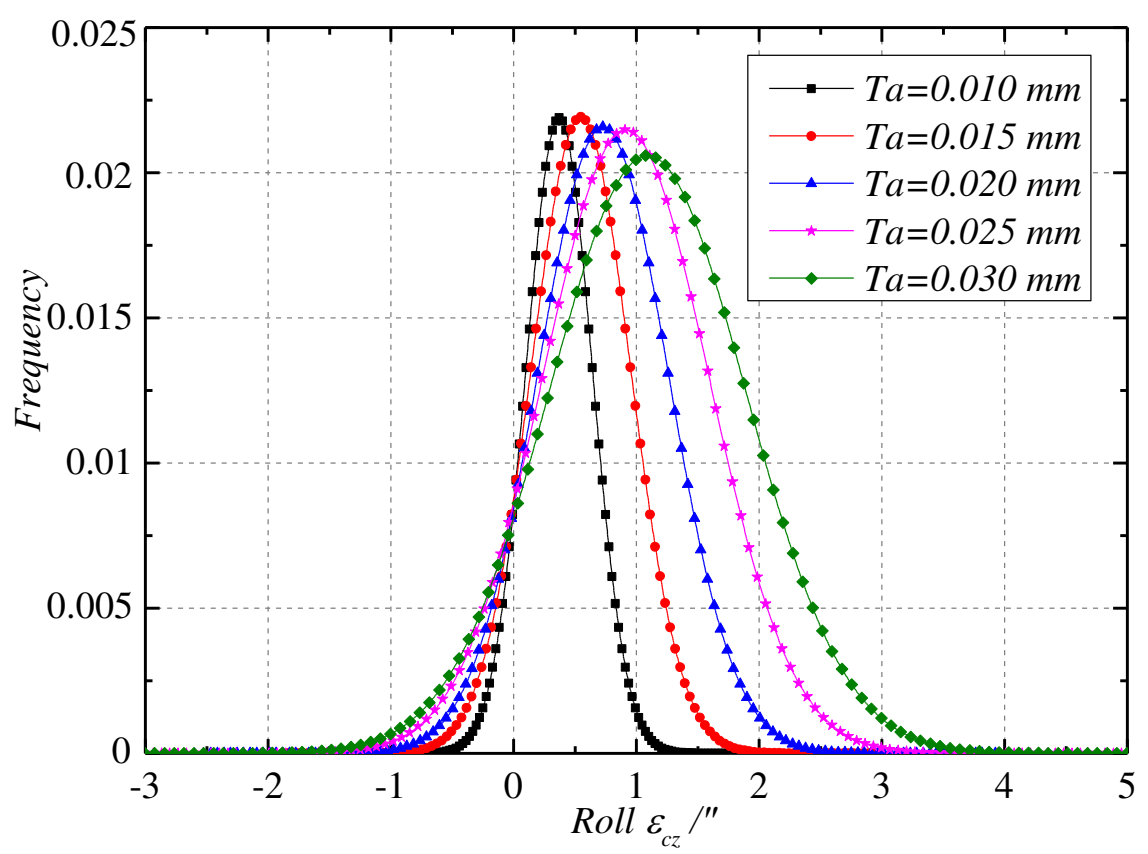

(e)

Fig. 21 Kinematic error of the linear axis under different flatness

(a) Straightness $\delta_{c x}$; (b) Straightness $\delta_{c y} ;$ (c) Pitch $\varepsilon_{c x}$; (d) Yaw $\varepsilon_{c y}$; (e) Roll $\varepsilon_{c z}$

\subsection{Effect of parallelism}

It can be seen from Fig. 22 that the parallelism error of the subordinate datum surface $C$ plays a key role in the kinematic error of the linear axis, especially the straightness error of surface yoz moving along $z$ axis, and the angular error of yaw. With the improvement of precision grade, five kinematic errors of the linear axis have a tendency to decrease, and the distribution range shrinks. When the parallelism tolerance decreases from $0.033 \mathrm{~mm}$ to 0.013 $\mathrm{mm}$, the straightness error of surface $y o z$ of the linear axis decreases from $0.03 \mathrm{~mm}$ to 0.01 $\mathrm{mm}$, and the angular error of roll decreases from $8 "$ to $3 "$, these results show a great change. However, the other three kinematic errors do not change obviously. 


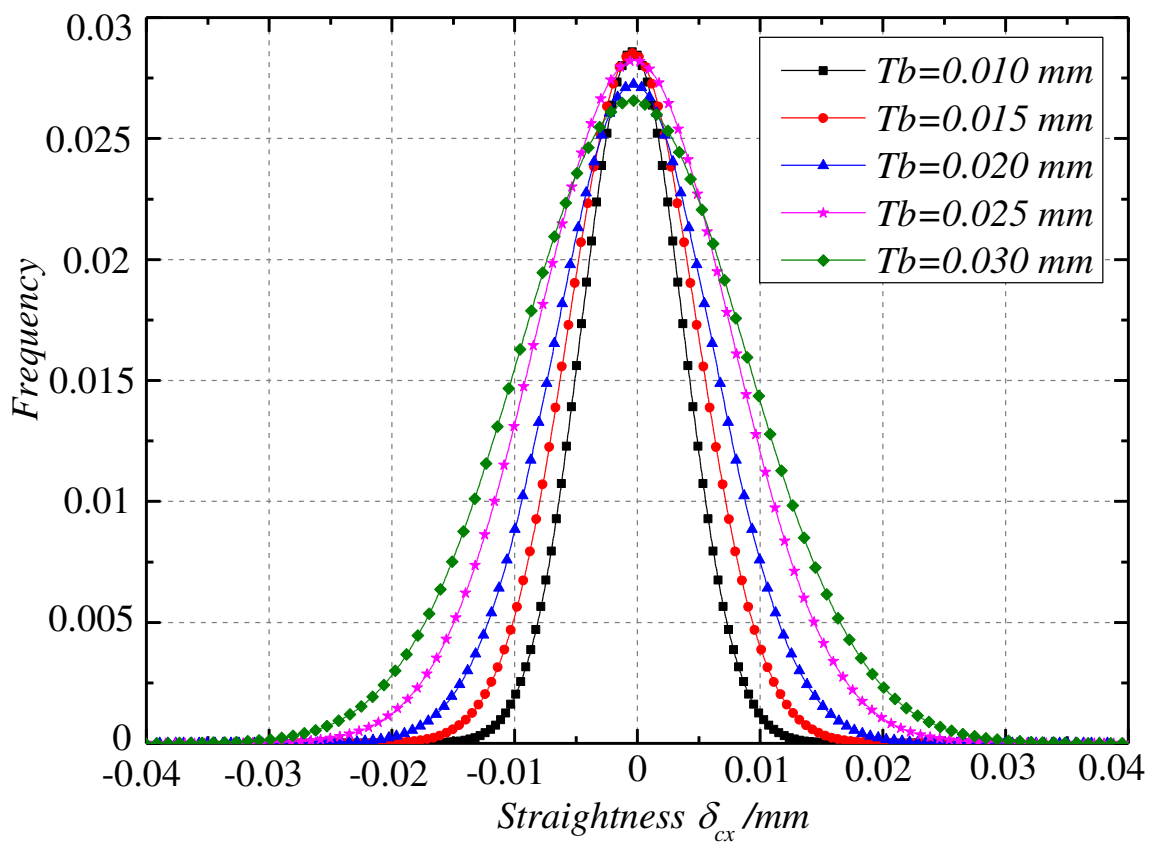

(a)

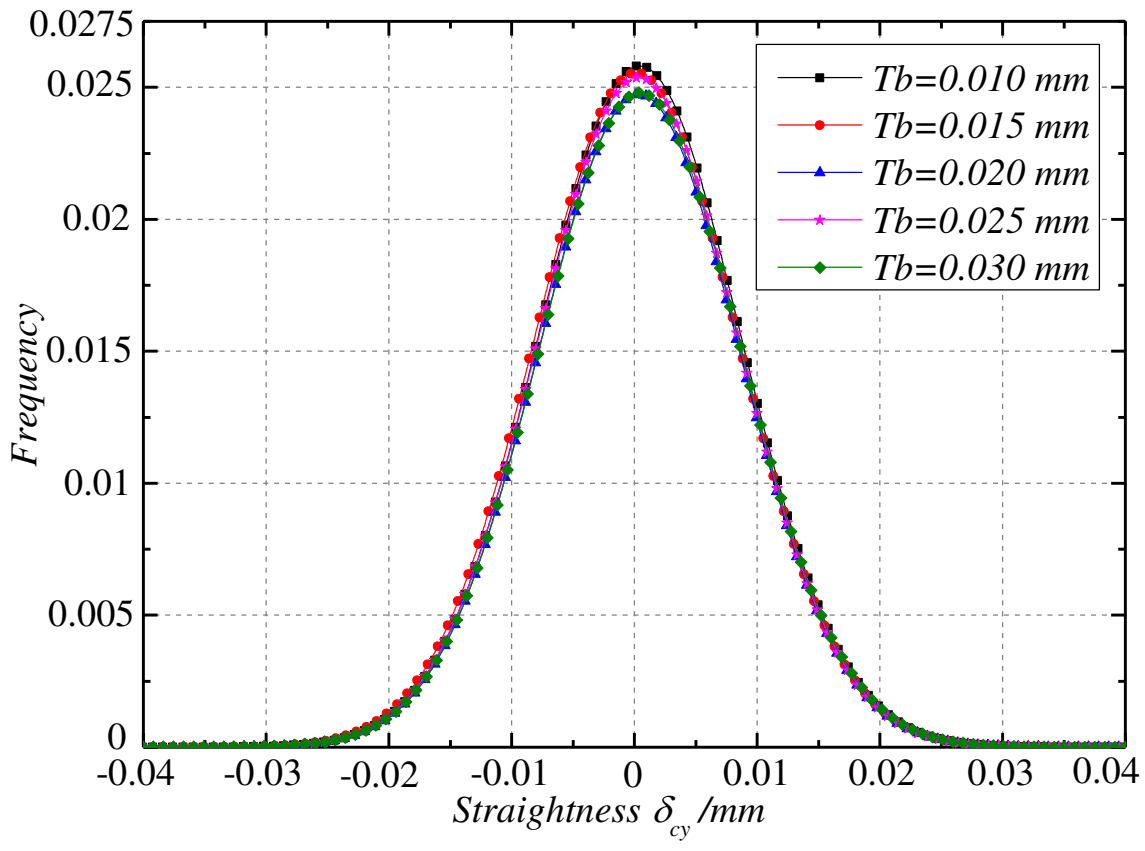

(b) 


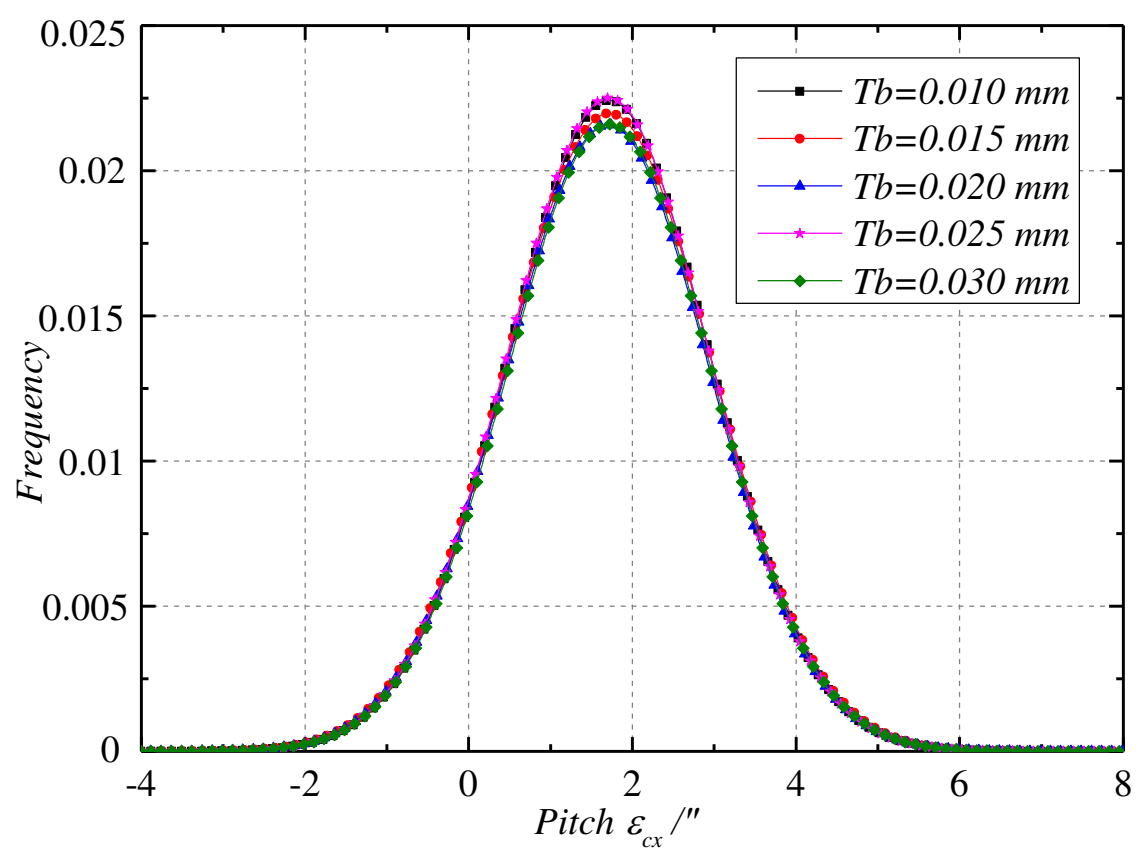

(c)

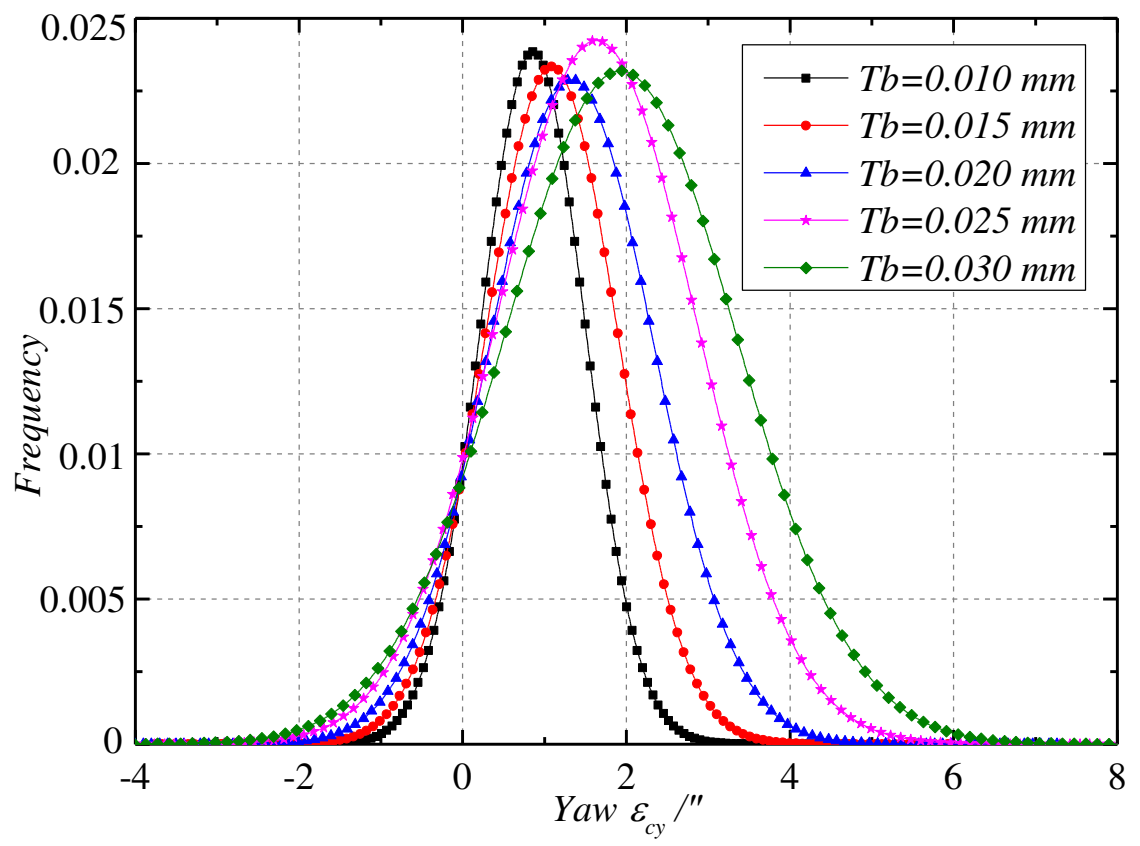

(d) 


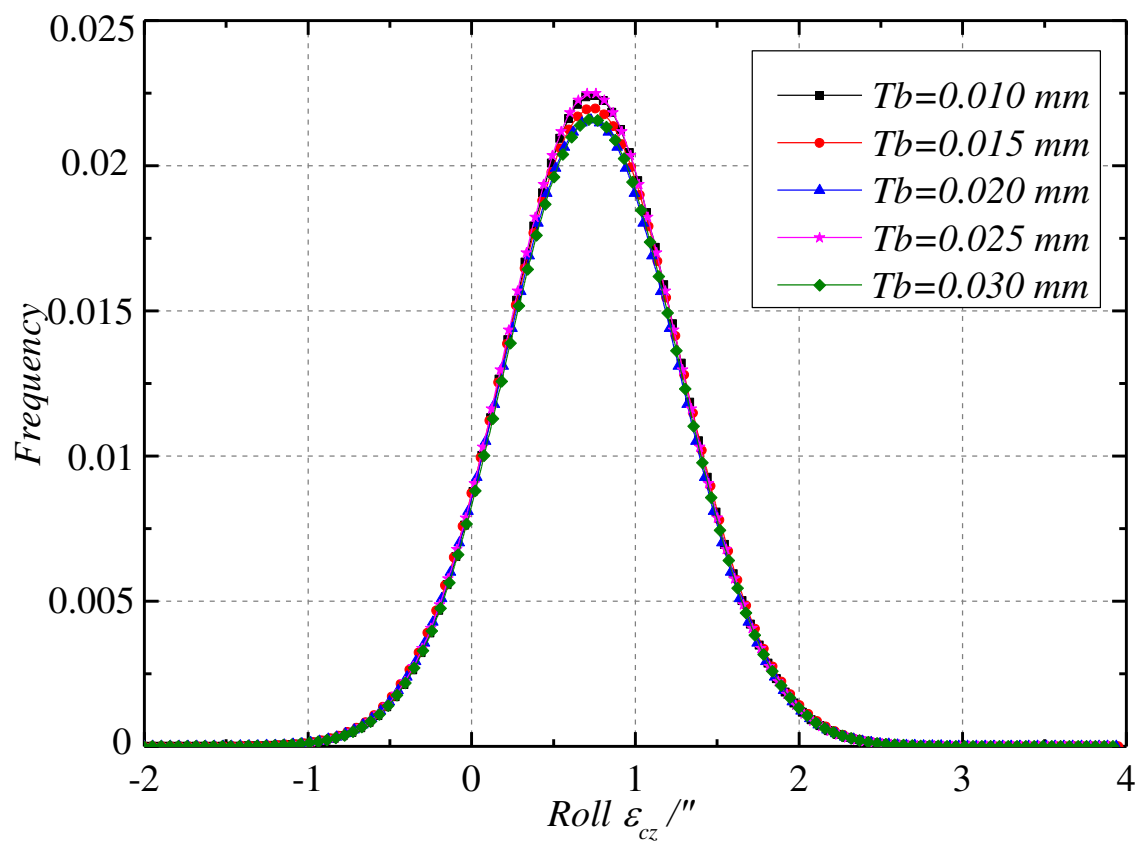

(e)

Fig. 22 Kinematic error of the linear axis under different parallelism

(a) Straightness $\delta_{c x}$; (b) Straightness $\delta_{c y} ;$ (c) Pitch $\varepsilon_{c x}$; (d) Yaw $\varepsilon_{c y}$; (e) Roll $\varepsilon_{c z}$

\subsection{Effect of perpendicularity}

As shown in Fig. 23, the influence trend of perpendicularity on the kinematic error of the linear axis is similar to that of parallelism, both have a great influence on the straightness error of surface yoz moving along $z$ axis, and the angular error of yaw. With the improvement of precision grade, five kinematic errors of the linear axis have a tendency to decrease, and the distribution range shrinks. Especially, the straightness error of surface yoz moving along $z$ axis, and the angular error of yaw show obvious changes. When the perpendicularity tolerance decreases from $0.03 \mathrm{~mm}$ to $0.01 \mathrm{~mm}$, the straightness error of surface yoz moving along $z$ axis decreases from $0.035 \mathrm{~mm}$ to $0.018 \mathrm{~mm}$, and the angular error of yaw decreases from $7.5^{\prime \prime}$ to $3.5 "$, a series of changes happen. However, similar to the parallelism error, the other three kinematic errors do not have such an obvious change. 


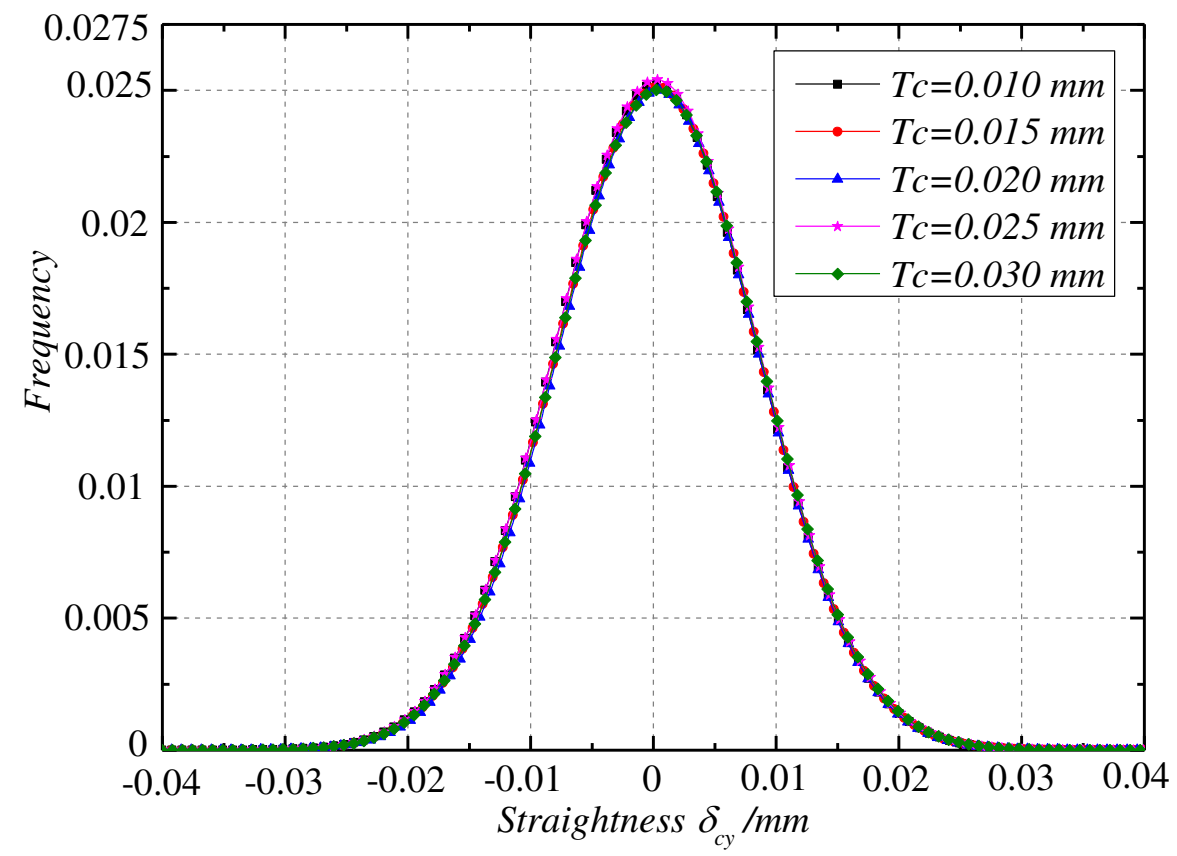

(a)

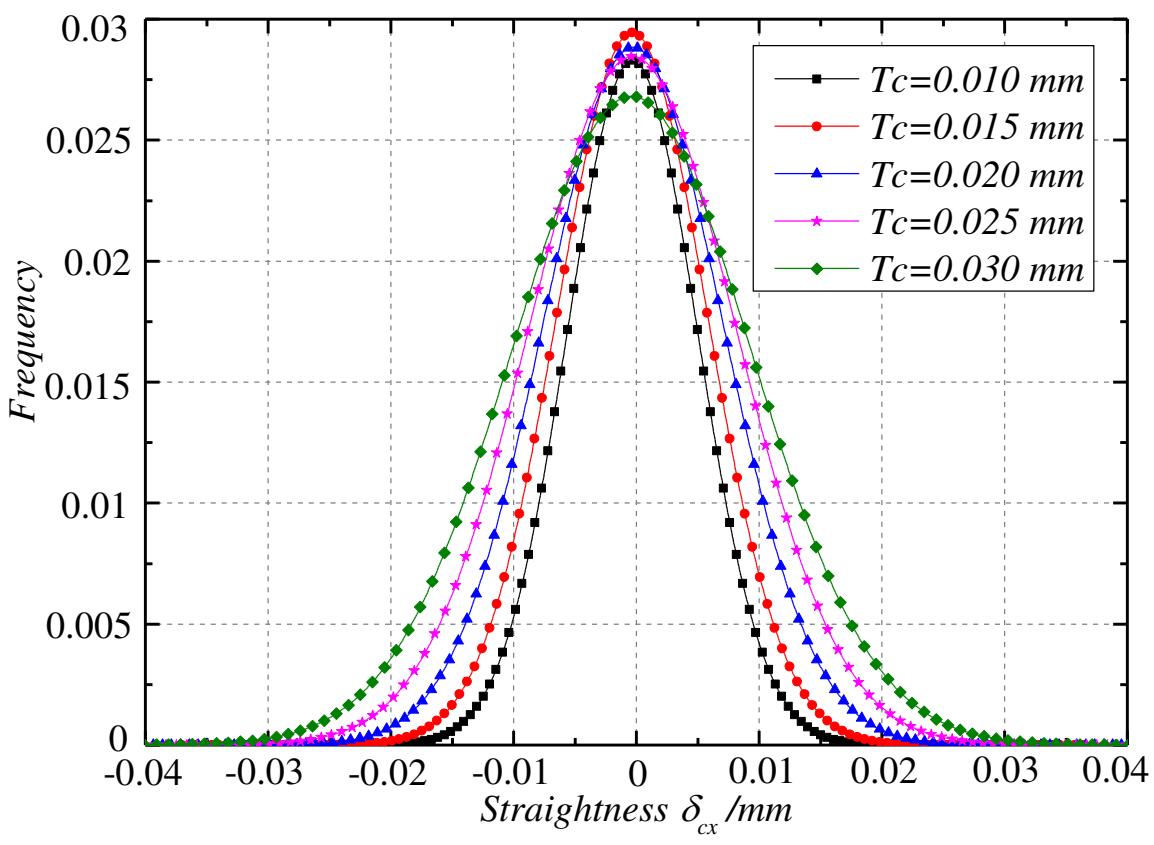

(b) 


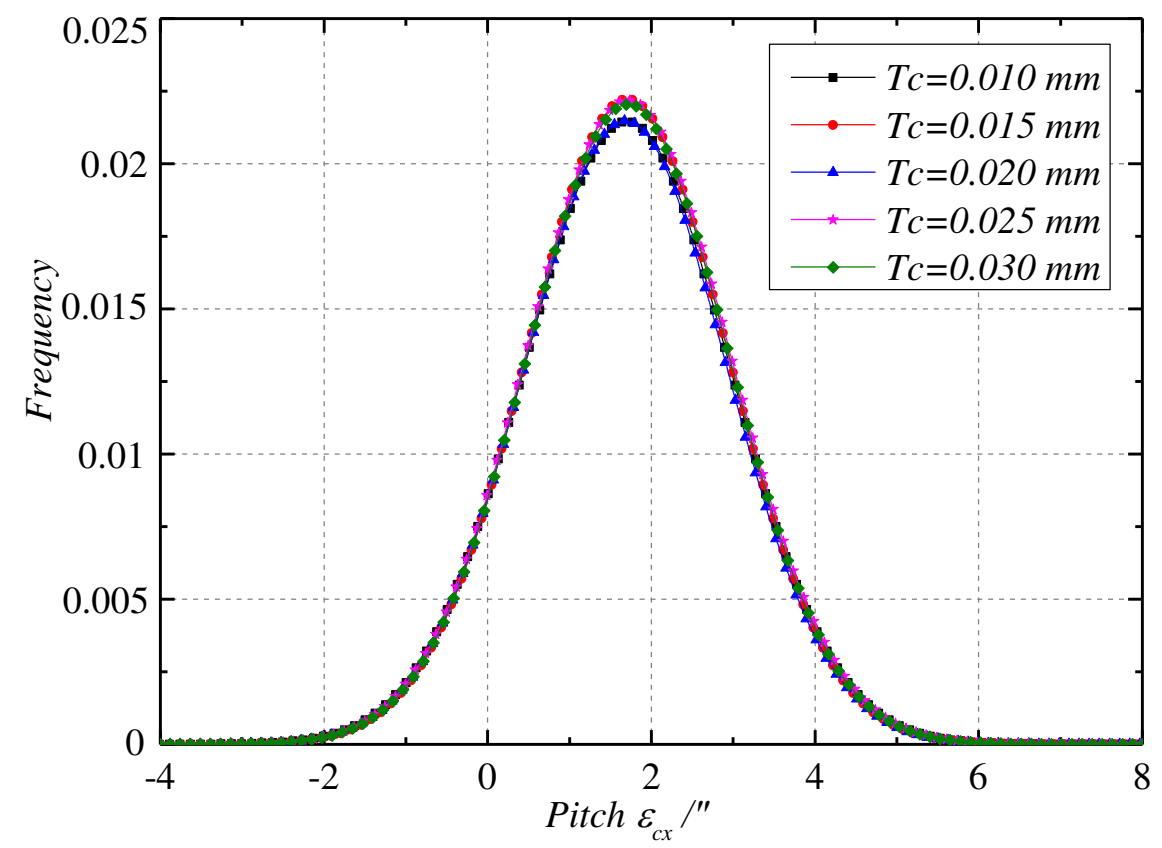

(c)

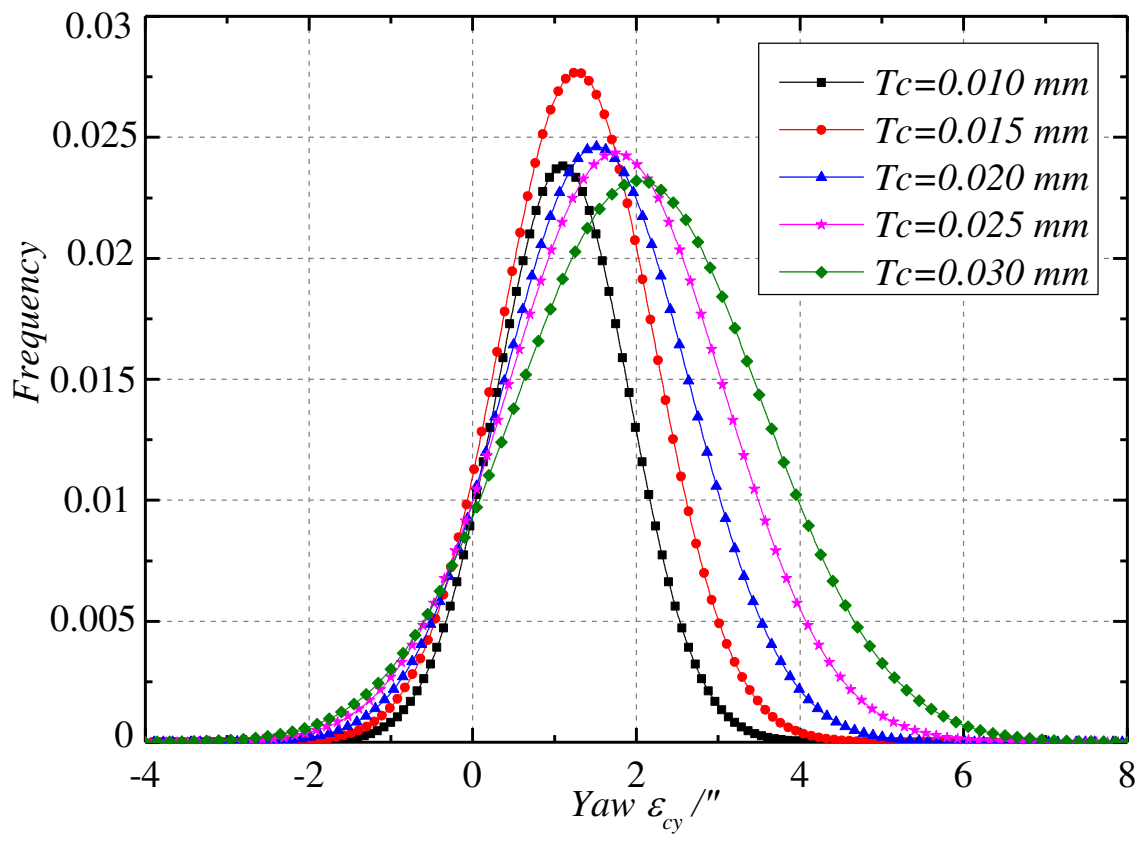

(d) 


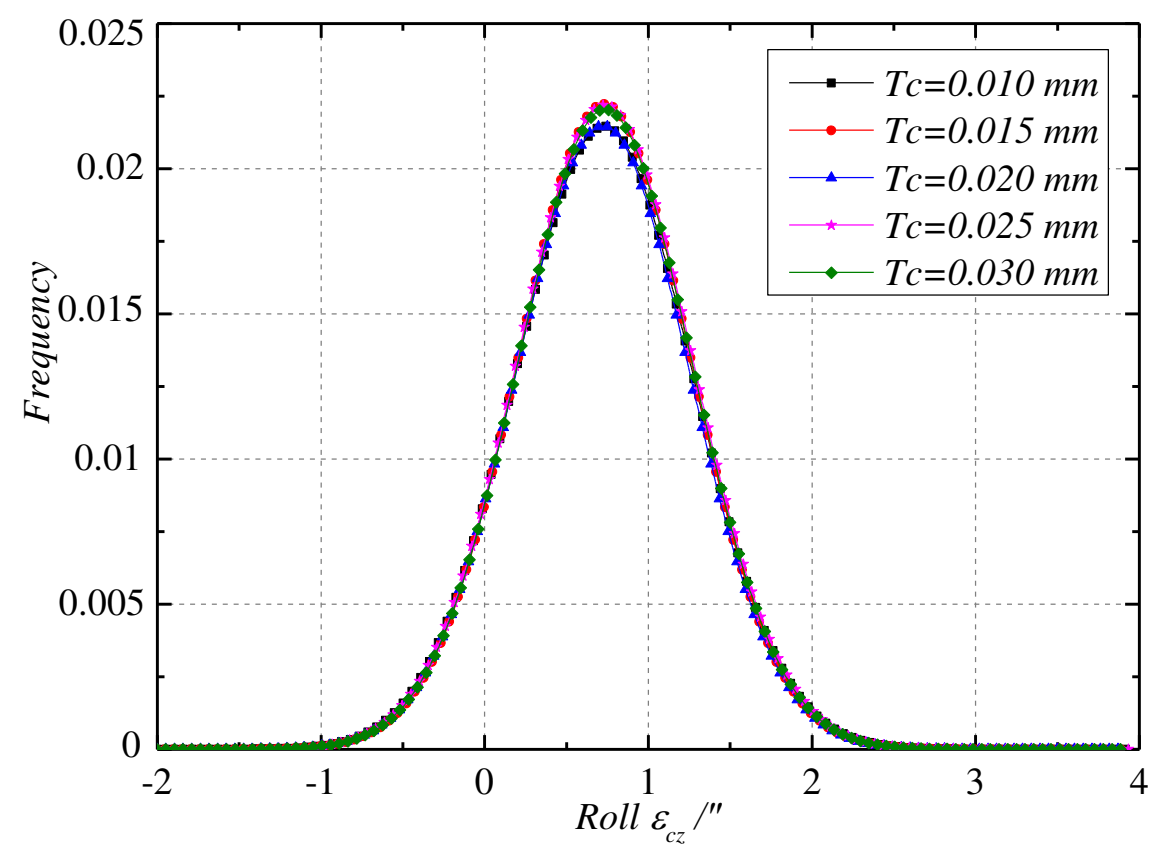

(e)

Fig. 23Kinematic error of the linear axis under different perpendicularity

(a) Straightness $\delta_{c x}$; (b) Straightness $\delta_{c y}$; (c) Pitch $\varepsilon_{c x}$; (d) Yaw $\varepsilon_{c y}$; (e) Roll $\varepsilon_{c z}$

\subsection{Effect of load}

\section{(1) Milling force}

It can be seen from Fig. 24 that the milling force has little effect on the kinematic error of the linear axis. When the milling force increases to $1200 \mathrm{~N}$, both the straightness error of surface yoz moving along $z$ axis and the angular error of yaw are larger than the other three kinematic errors, while the overall distribution range is not highly sensitive to the milling force. 


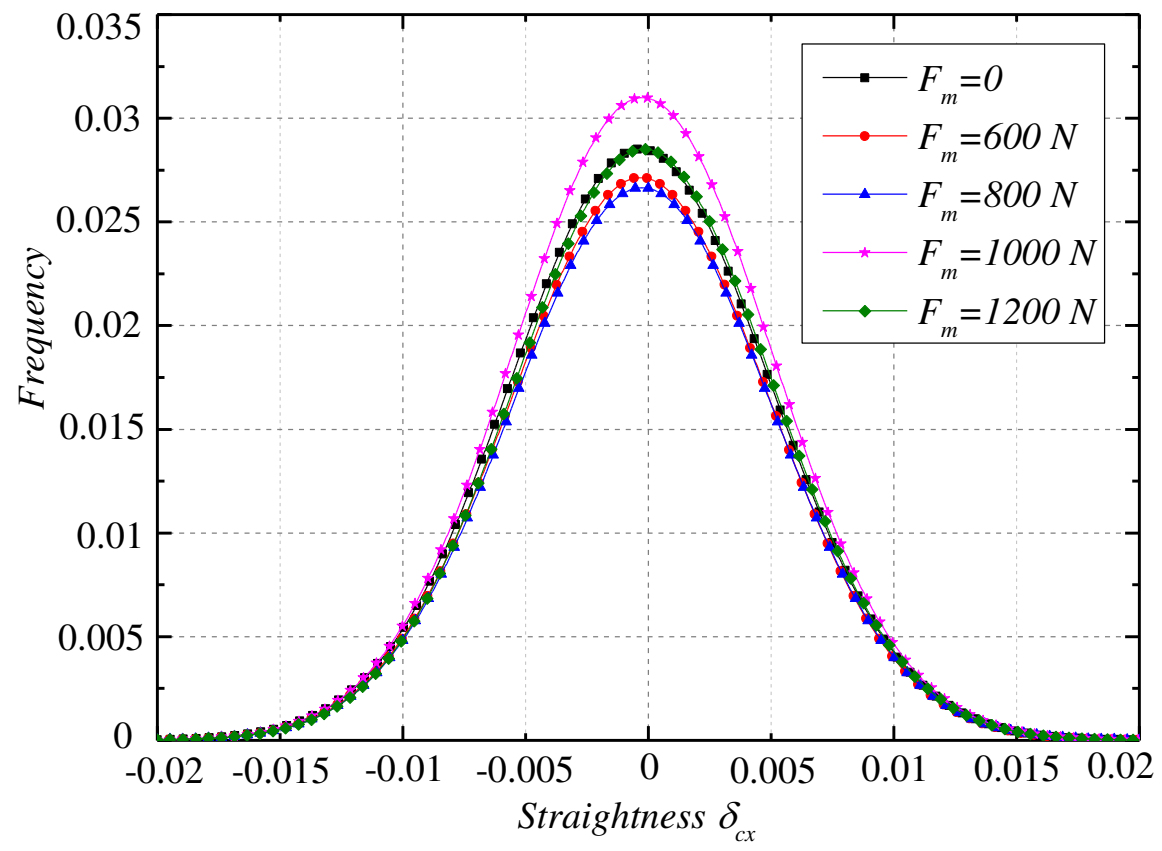

(a)

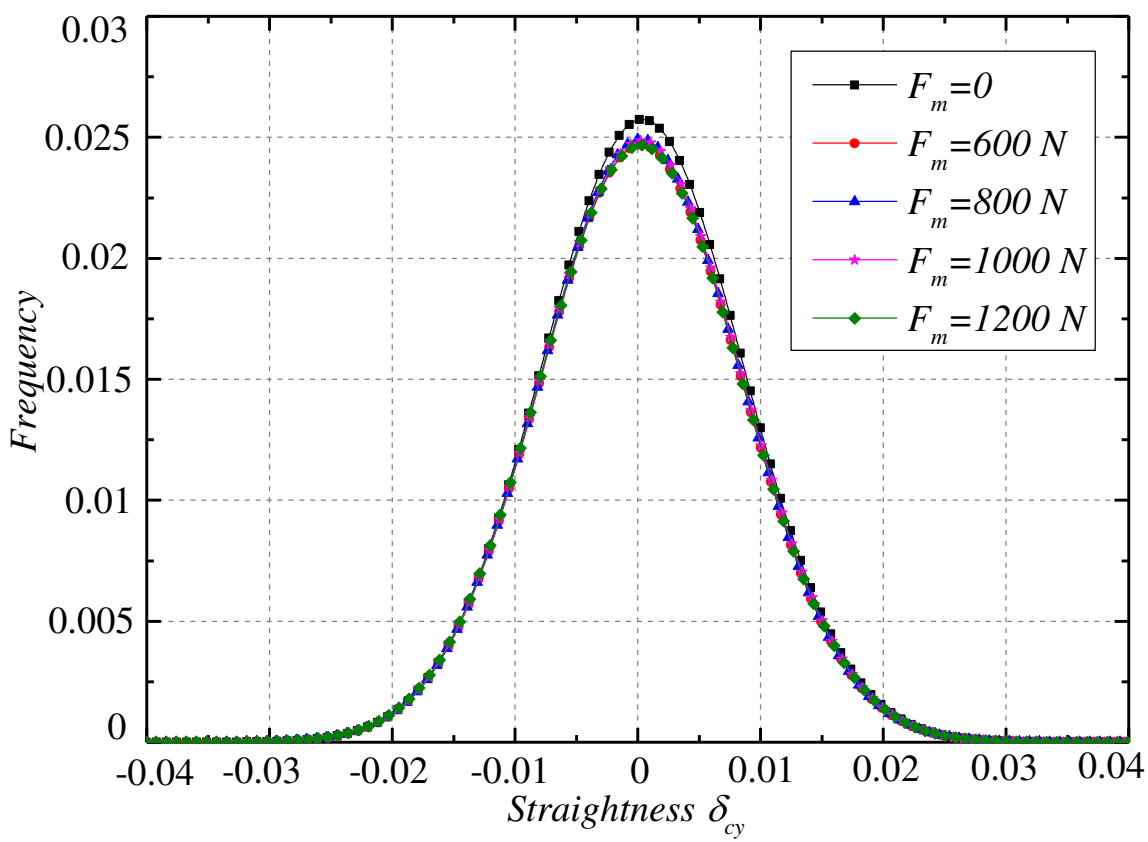

(b) 


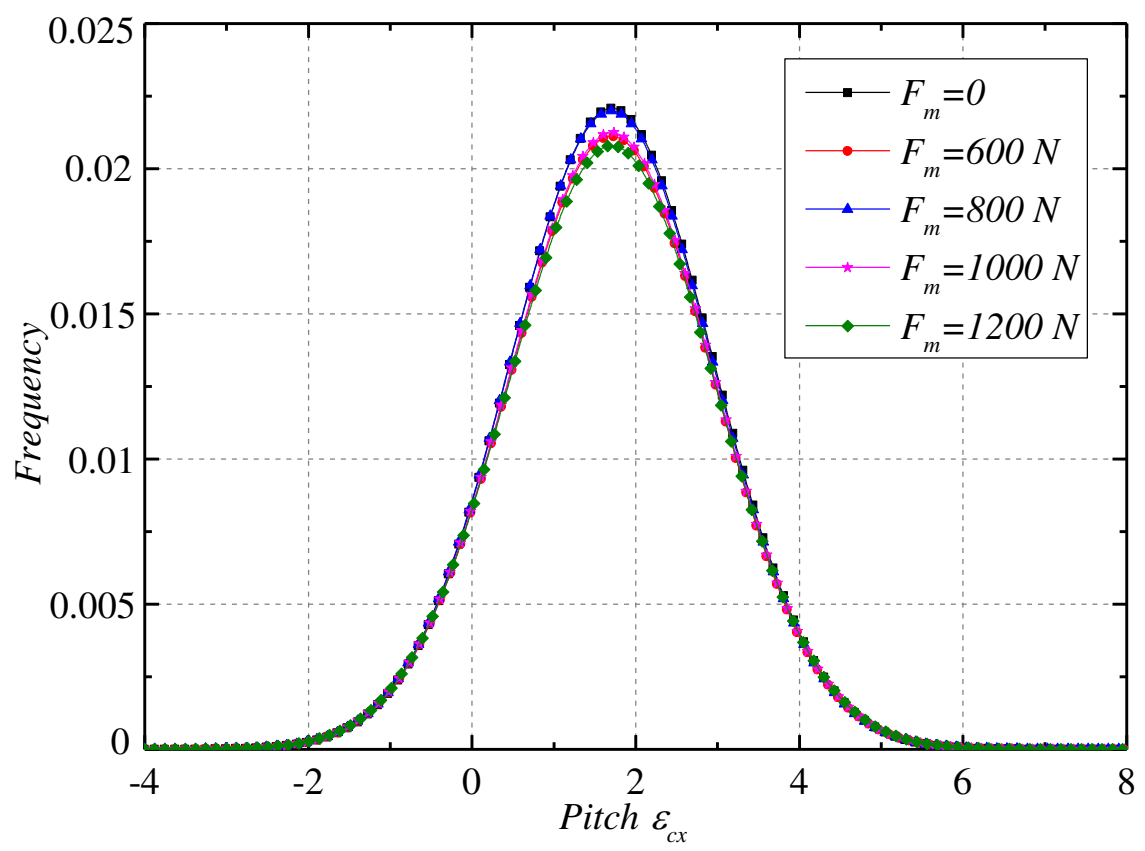

(c)

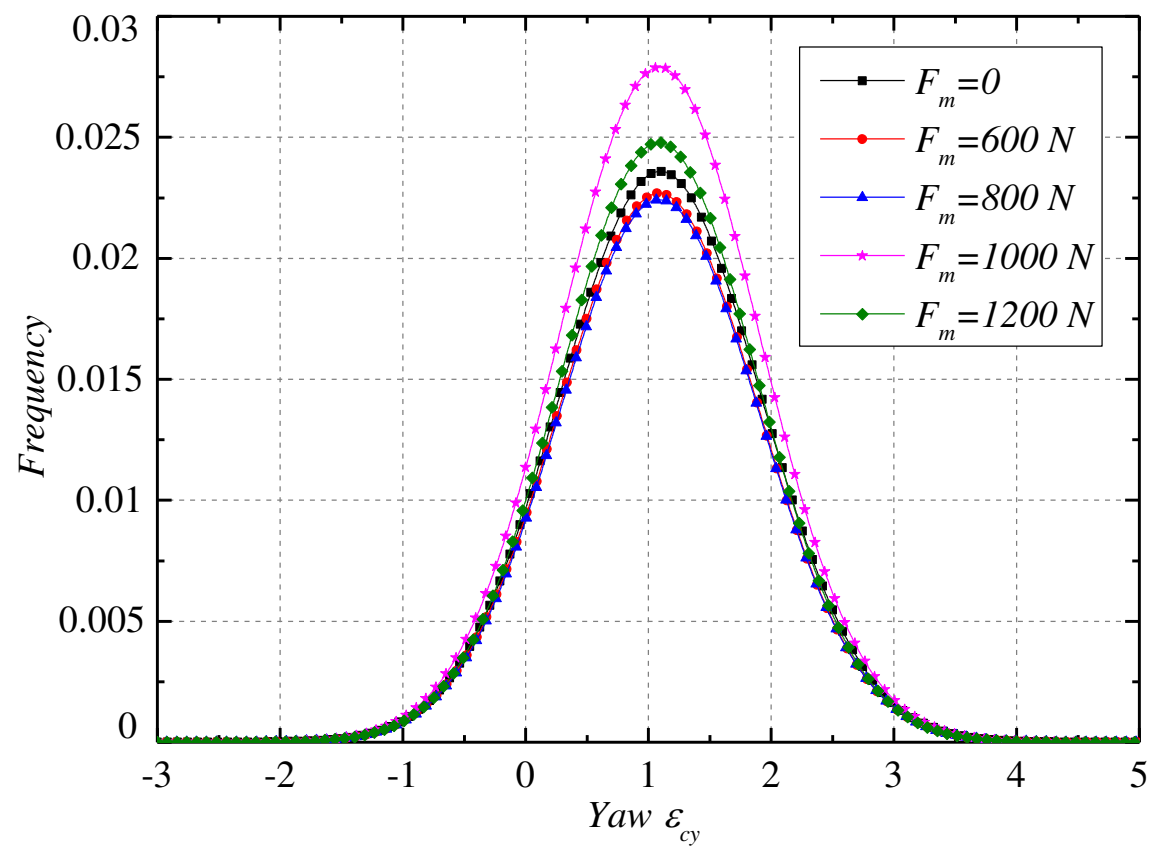

(d) 


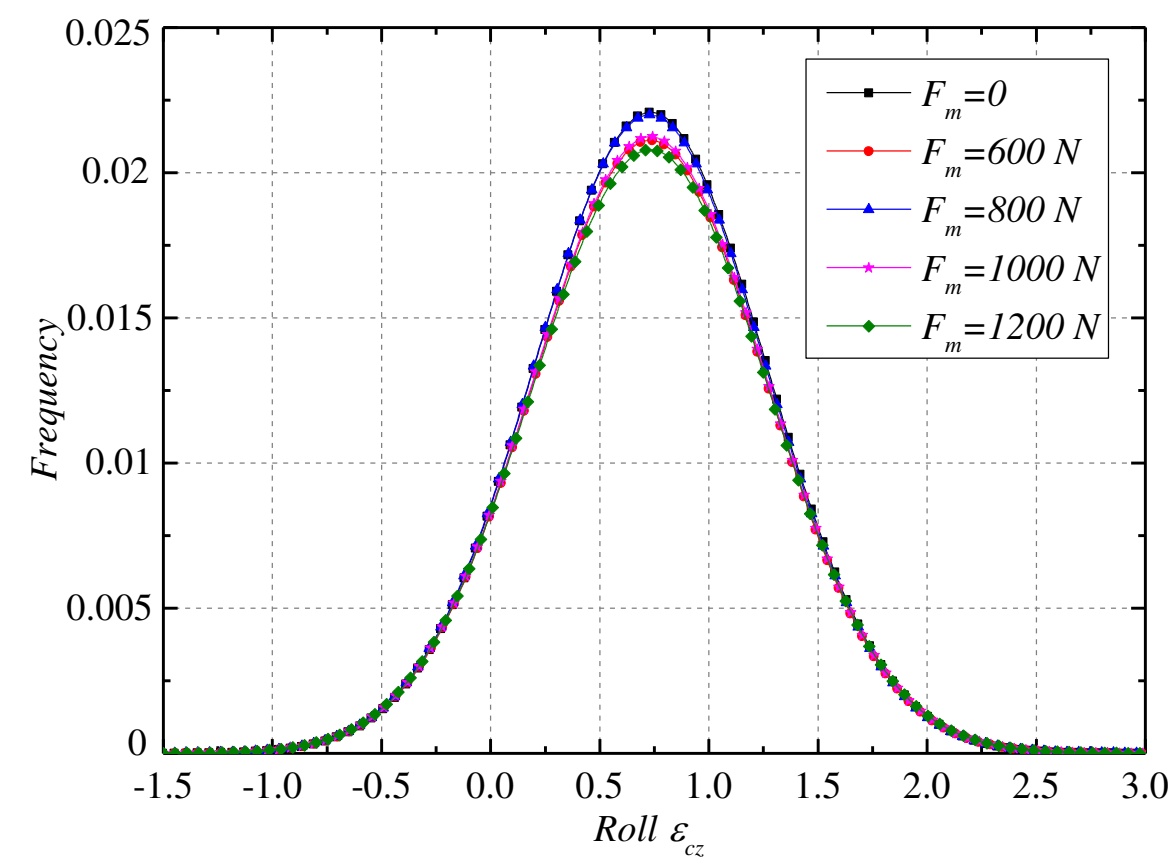

(e)

Fig. 24 Kinematic error of the linear axis under different milling force

(a) Straightness $\delta_{c x}$; (b) Straightness $\delta_{c y} ;$ (c) Pitch $\varepsilon_{c x}$; (d) Yaw $\varepsilon_{c y}$; (e) Roll $\varepsilon_{c z}$

\section{(2) Gravity}

As shown in Fig. 25, the workpiece gravity has almost no influence on the kinematic error of the linear axis. When the workpiece gravity increases form $1.60 \times 10^{4} \mathrm{~N}$ to $1.72 \times 10^{4} \mathrm{~N}$, the overall distribution range is not sensitive to the gravity. 


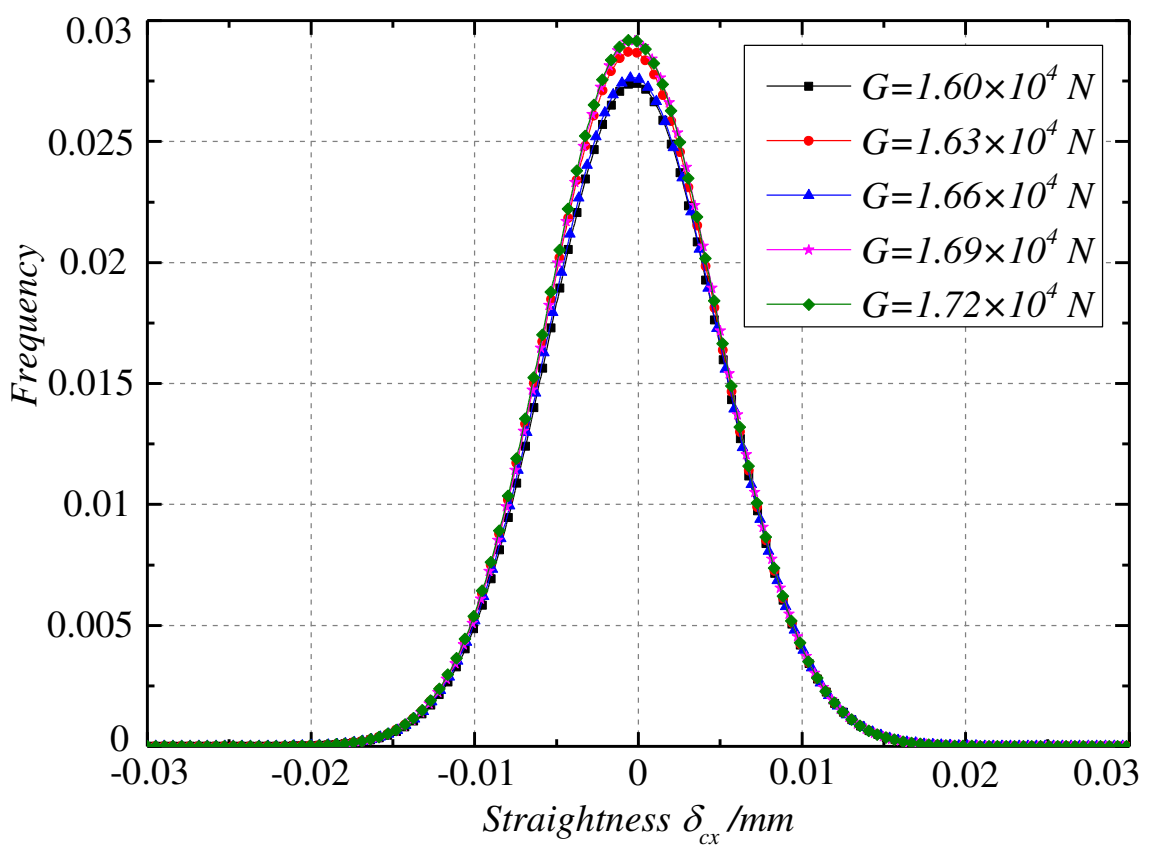

(a)

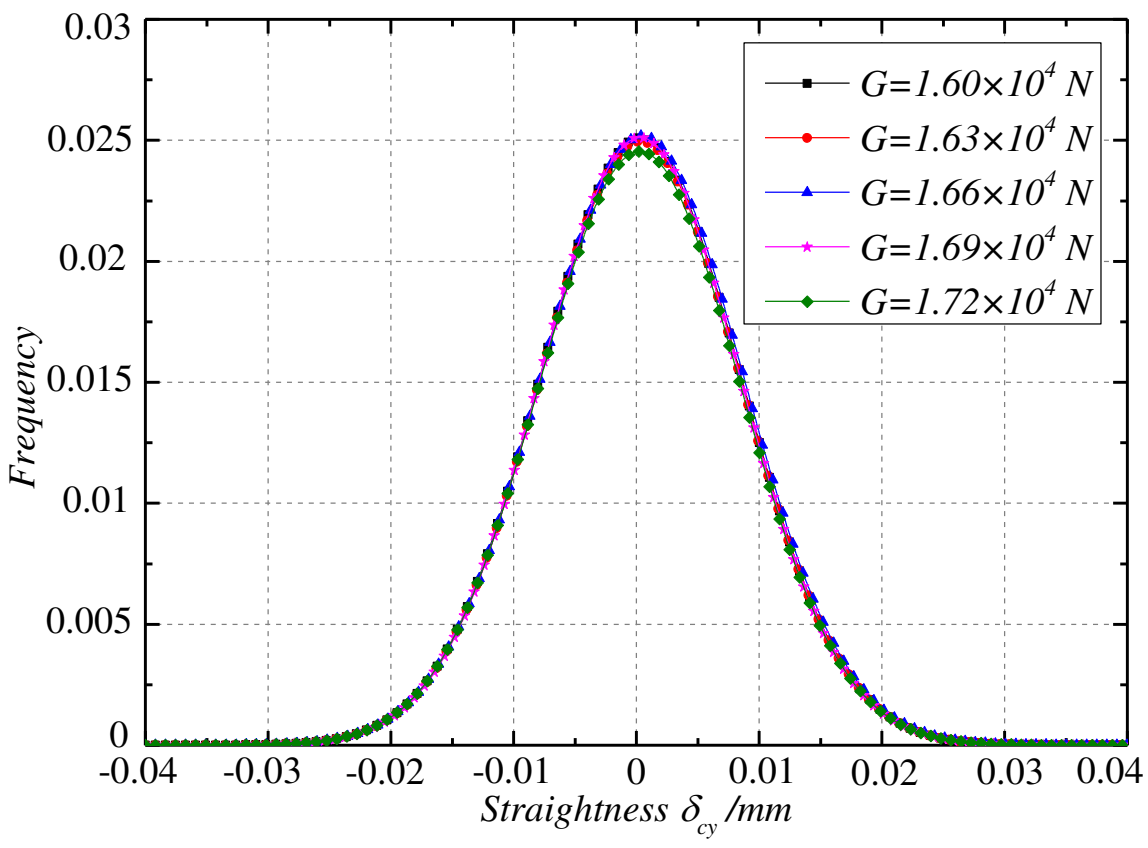

(b) 


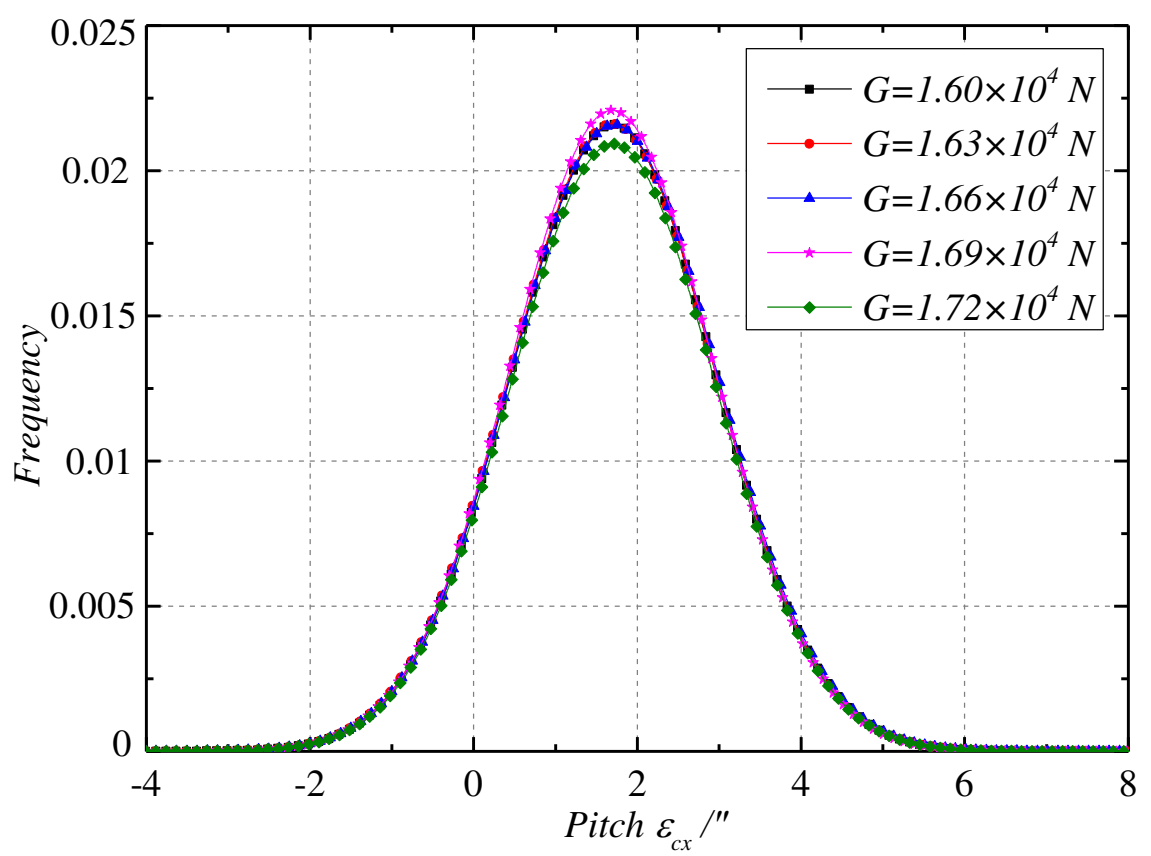

(c)

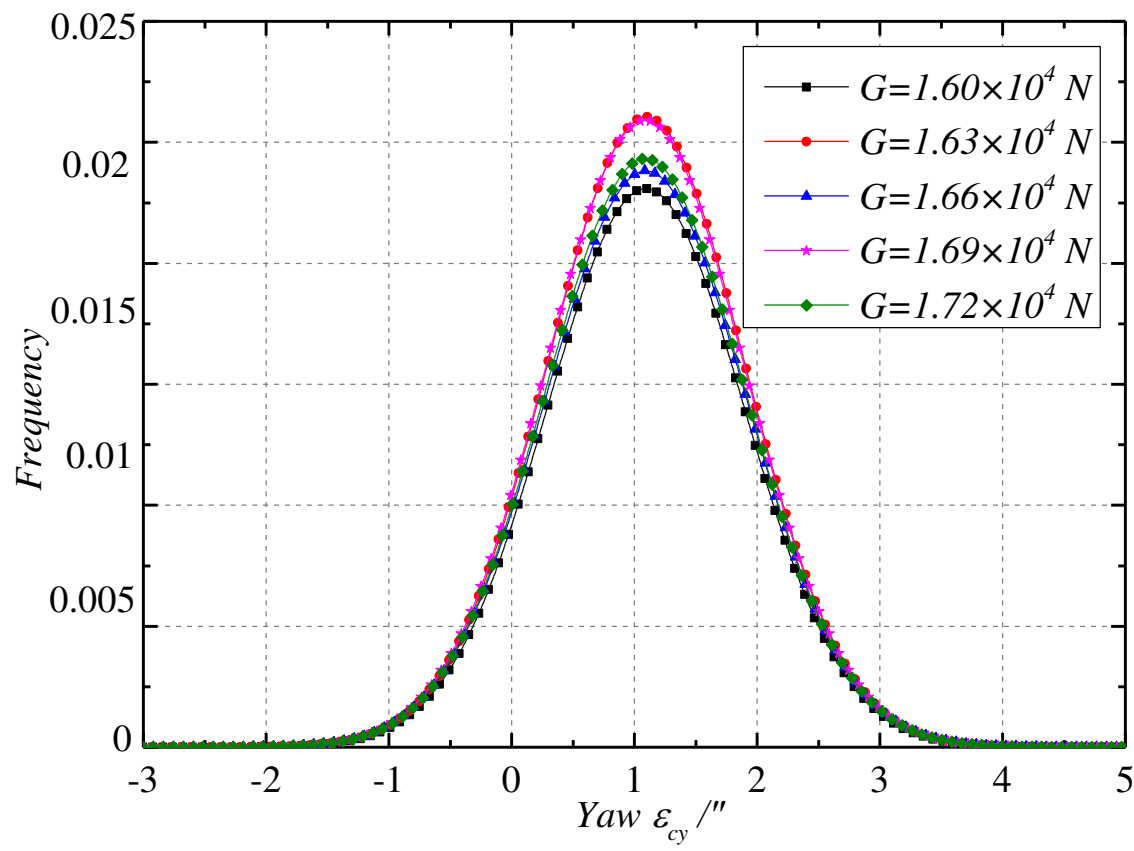

(d) 


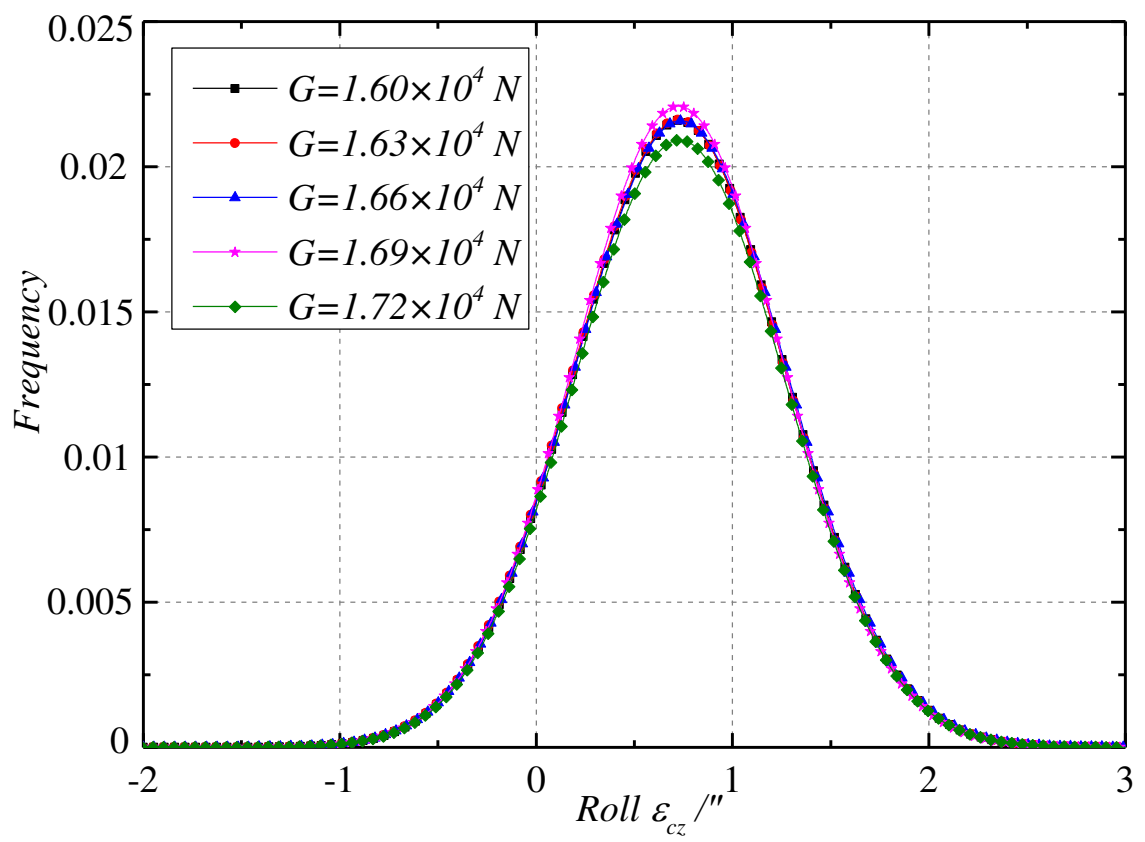

(e)

Fig. 25 Kinematic error of the linear axis under different gravity

(a) Straightness $\delta_{c x}$; (b) Straightness $\delta_{c y} ;$ (c) Pitch $\varepsilon_{c x}$; (d) Yaw $\varepsilon_{c y}$; (e) Roll $\varepsilon_{c z}$

According to the analysis of the effect factors in all the above figures, the effect degree comparison of effect factors on the kinematic error of the linear axis is shown in Table 5. As can be seen from the following Table, flatness is the most crucial effect factor, especially on the straightness $\delta_{c y}$ along yoz plane, Pitch $\varepsilon_{c x}$ and Roll $\varepsilon_{c z}$. Then, parallelism and perpendicularity are also the key effect factors on the straightness $\delta_{c x}$ along xoz plane and Yaw $\varepsilon_{c y}$. In addition, the effect of gravity and milling force on the kinematic error of the linear axis is relatively smaller. Therefore, based on the analysis results, some guidelines can be provided for the design and machining of the linear axis of high-end machine tool. 
Table 4 Effect analysis on the kinematic error of linear axis

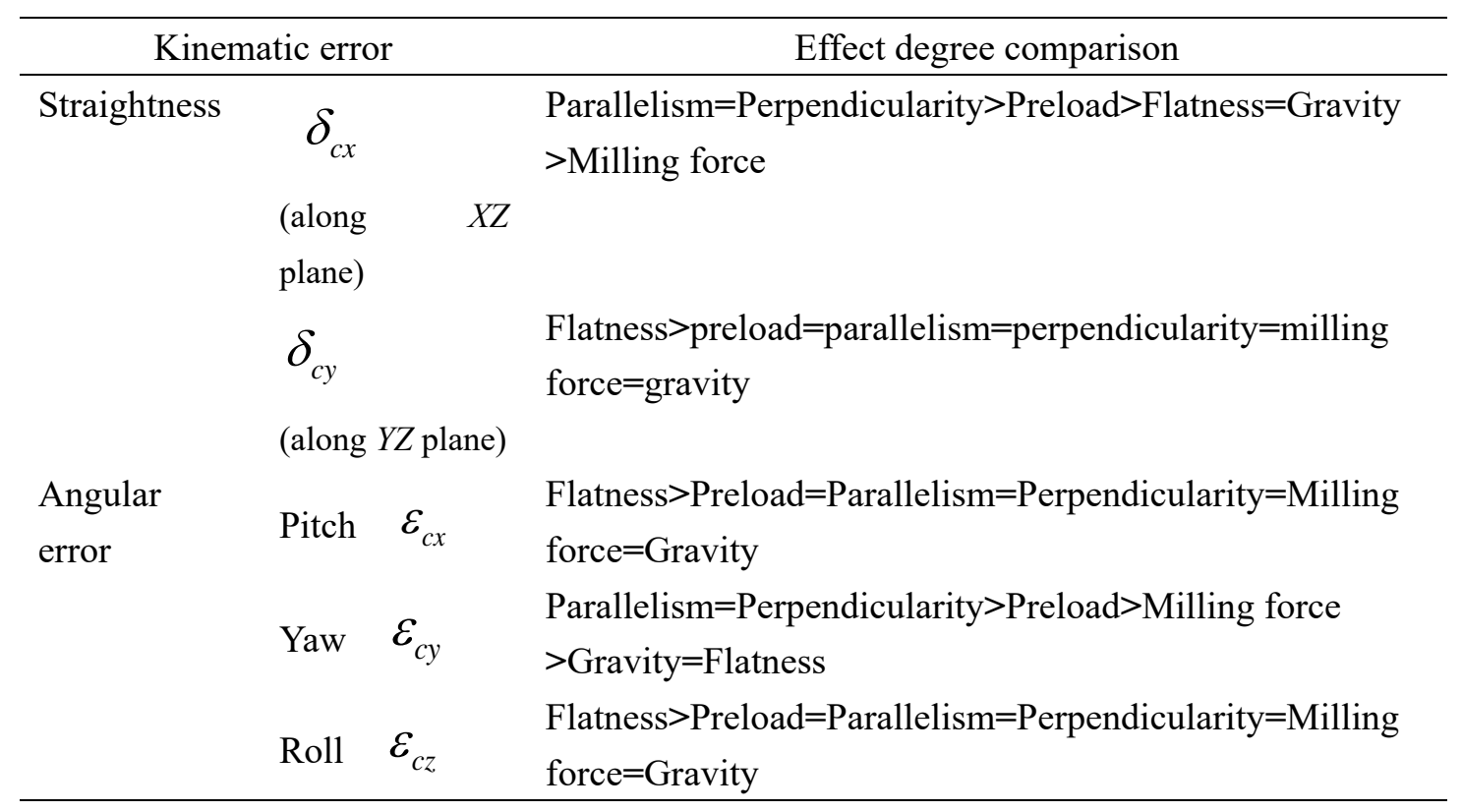

\section{Conclusions}

This paper presented a systematic error equivalence model of slider, aiming to predict the kinematic errors of the linear axis of machine tool. The proposed methodology contains: (i) the error equivalence method for the kinematic error of slider; (ii) the vector summation method for the kinematic error of the linear axis. The verification experiment shows a great consistence. It indicates that the proposed model is accurate and effectiveness. Through the effect analysis of the kinematic error of the linear axis, it is obvious that the influence of flatness is relatively greater among all factors. In summary, the proposed model can be used to obtain the kinematic error of the linear axis at the design stage. Furthermore, the quantitative guidance can be provided in the design and manufacturing of the linear axis of machine tool. It improves the design and manufacturing efficiency significantly.

In future study, we will add the study of the surface topography effect of linear guide rail on the kinematic error of the linear axis, and further obtain the accuracy retention rules of the linear axis, aiming to predict the operation life of machine tool.

\section{DECLARATIONS}

(1) Ethical Approval 
Yes.

(2) Consent to Participate

Yes, the authors consent to participate.

(3) Consent to Publish

Yes, the authors consent to publish.

(4) Authors Contributions

Xinxin LI: Conceptualization, Methodology, Software, Validation, Visualization, Writing original draft, Writing - review \& editing.

Zhimin LI: Conceptualization, Methodology, Validation, Visualization, Supervision, Writing -

Writing -review \& editing.

Sun JIN: Conceptualization, Supervision.

Jichang ZHANG: Conceptualization, Supervision.

Siyi DING: Conceptualization, Software

Zhihua NIU: Conceptualization, Visualization

(5) Funding

This work is provided the financial support by the National Science and Technology major project (No. 2017ZX04016001) and the National Natural Science Foundation of China (No. 51775346).

(6) Competing Interests

The authors declare that they have no known competing interests or personal relationships that could have appeared to influence the work reported in this paper.

(7) Availability of data and materials

Not applicable.

\section{References}

1. He G, Sun G, Zhang H, Huang C and Zhang D (2017) Hierarchical error model to estimate motion error of linear motion bearing table. Int. J. Adv. Manuf. Technol. 93(5-8): 1915-1927. 10.1007/s00170-017-0635-0

2. Zha J, Lv D, Jia Q and Chen Y (2016) Motion straightness of hydrostatic guideways considering the ratio of pad center spacing to guide rail profile error wavelength. Int. J. Adv. Manuf. Technol. 
82(9-12): 2065-2073. 10.1007/s00170-015-7515-2

3. Xue F, Zhao W, Chen Y and Wang Z (2012) Research on error averaging effect of hydrostatic guideways. Precis. Eng.-J. Int. Soc. Precis. Eng. Nanotechnol. 36(1): 84-90. 10.1016/j.precisioneng.2011.07.007

4. Fan J, Tao H, Wu C, Pan R, Tang Y and Li Z (2018) Kinematic errors prediction for multi-axis machine tools' guideways based on tolerance. Int. J. Adv. Manuf. Technol. 98(5-8): 1131-1144. 10.1007/s00170-018-2335-9

5. Zhong X, Liu H, Mao X, Li B and He S (2019) Influence and error transfer in assembly process of geometric errors of a translational axis on volumetric error in machine tools. Measurement 140450-461. 10.1016/j.measurement.2019.04.032

6. Zhang P, Chen Y, Zhang C, Zha J and Wang T (2018) Influence of geometric errors of guide rails and table on motion errors of hydrostatic guideways under quasi-static condition. Int. J. Mach. Tools Manuf. 12555-67. https://doi.org/10.1016/j.ijmachtools.2017.10.006

7. Chlebus E and Dybala B (1999) Modelling and calculation of properties of sliding guideways. Int. J. Mach. Tools Manuf. 39(12): 1823-1839. https://doi.org/10.1016/S0890-6955(99)00041-3

8. Majda P (2012) Modeling of geometric errors of linear guideway and their influence on joint kinematic error in machine tools. Precis. Eng.-J. Int. Soc. Precis. Eng. Nanotechnol. 36(3): 369-378. 10.1016/j.precisioneng.2012.02.001

9. Shimizu S (1998) Stiffness analysis of precision machinery elements. stiffness analysis of linear motion rolling guide. Journal of the Japan Society for Precision Engineering 64(11): 1573-1576. $10.2493 / \mathrm{jj}$ spe.64.1573

10. Ohta H and Tanaka K (2010) Vertical stiffnesses of preloaded linear guideway type ball bearings incorporating the flexibility of the carriage and rail. J. Tribol.-Trans. ASME 132(1): 1-9. $10.1115 / 1.4000277$

11. Jeong J, Kang E and Jeong J (2014) Equivalent stiffness modeling of linear motion guideways for stage systems. Int. J. Precis. Eng. Manuf. 15(9): 1987-1993. 10.1007/s12541-014-0555-y

12. Zou HT and Wang BL (2015) Investigation of the contact stiffness variation of linear rolling guides due to the effects of friction and wear during operation. Tribol. Int. 92472-484. 10.1016/j.triboint.2015.07.005

13. Ma Y and Li Y (2019) Motion error of rolling guide based on uncertainty of geometric error. Chin. J. Mech. Eng. 55(5): 11-18. 10.3901/JME.2019.05.011

14. Khim G, Oh JS and Park CH (2014) Analysis of 5-DOF motion errors influenced by the guide rails of an aerostatic linear motion stage. Int. J. Precis. Eng. Manuf. 15(2): 283-290. $10.1007 / \mathrm{s} 12541-014-0336-7$

15. Khim G, Park CH and Oh JS (2015) A method of calculating motion error in a linear motion bearing stage. The Scientific World Journal 20151-10. 10.1155/2015/696417

16. Ekinci TO and Mayer JRR (2007) Relationships between straightness and angular kinematic errors in machines. Int. J. Mach. Tools Manuf. 47(12-13): 1997-2004. 10.1016/j.ijmachtools.2007.02.002

17. Ekinci TO, Mayer JRR and Cloutier GM (2009) Investigation of accuracy of aerostatic guideways. Int. J. Mach. Tools Manuf. 49(6): 478-487. 10.1016/j.ijmachtools.2009.01.001

18. Khim G, Park CH, Shamoto E and Kim SW (2011) Prediction and compensation of motion 
accuracy in a linear motion bearing table. Precis. Eng.-J. Int. Soc. Precis. Eng. Nanotechnol. 35(3): 393-399. 10.1016/j.precisioneng.2010.12.006

19. Barus C (1900) A treatise on the theory of screws. Science 12(313): 1001-1003.

20. Bourdet P, Mathieu L, Lartigue C and A B (1996) The concept of the small displacement torsor in metrology. Advances in Applied Mathematics 40110-122. http://dx.doi.org/

21. Ding S, Jin S, Li Z and Chen H (2019) Multistage rotational optimization using unified Jacobian-torsor model in aero-engine assembly. Proc. Inst. Mech. Eng. Part B-J. Eng. Manuf. 233(1): 251-266. 10.1177/0954405417703431

22. Alain D, Walid G and Luc LR (2003) Application of a unified jacobian-torsor model for tolerance analysis. J. Comput. Inf. Sci. Eng. 3(1): 2-14. 10.1115/1.1573235

23 Jin S., Chen H, Li Z andLai X (2015) A small displacement torsor model for 3D tolerance analysis of conical structures, Proc. Inst. Mech. Eng. Part C-J. Eng. Mech. Eng. Sci. 229(14) 2514-2523.

24. Zhong X, Yang R and Zhou B (2003) Accuracy analysis of assembly success rate with Monte Carlo simulations. J. DongHua Uni. 20(4): 128-131. 10.3969/j.issn.1672-5220.2003.04.027

25. Jia Z and Wang F (2011) Foundation of machine manufacturing technology, Science Press, Beijing

26. Johnson KL (1985) Contact mechanics, Cambridge: Cambridge University Press

27. Tedric A. H and Michael N. K (1991) Rolling bearing analysis, New York: Wiley

28. Murray RM, Li Z and Sastry SS (1994) A mathematical introduction to robotic manipulation, CRC Press

29. Goswami DY (2004) The CRC Handbook of Mechanical Engineering, CRC press

30. Yu W, Wang DL, Wang Z, Dong HM, Yu DS (2016) The kinematic invariants in testing error motion of machine tool linear axes. Mechanism and Machine Science 408: 1525-1540. 10.1007/978-981-10-2875-5_121

31. ISO 230-1:2012, Test code for machine tools_part 1: geometric accuracy of machines operating under no-load or quasi-static conditions, pp 1-11, (2012)

32. Pawełko P, Berczyński S and Grządziel Z (2014) Modeling roller guides with preload. Arch. Civ. Mech. Eng. 14(4): 691-699. 10.1016/j.acme.2013.12.002 
Figures

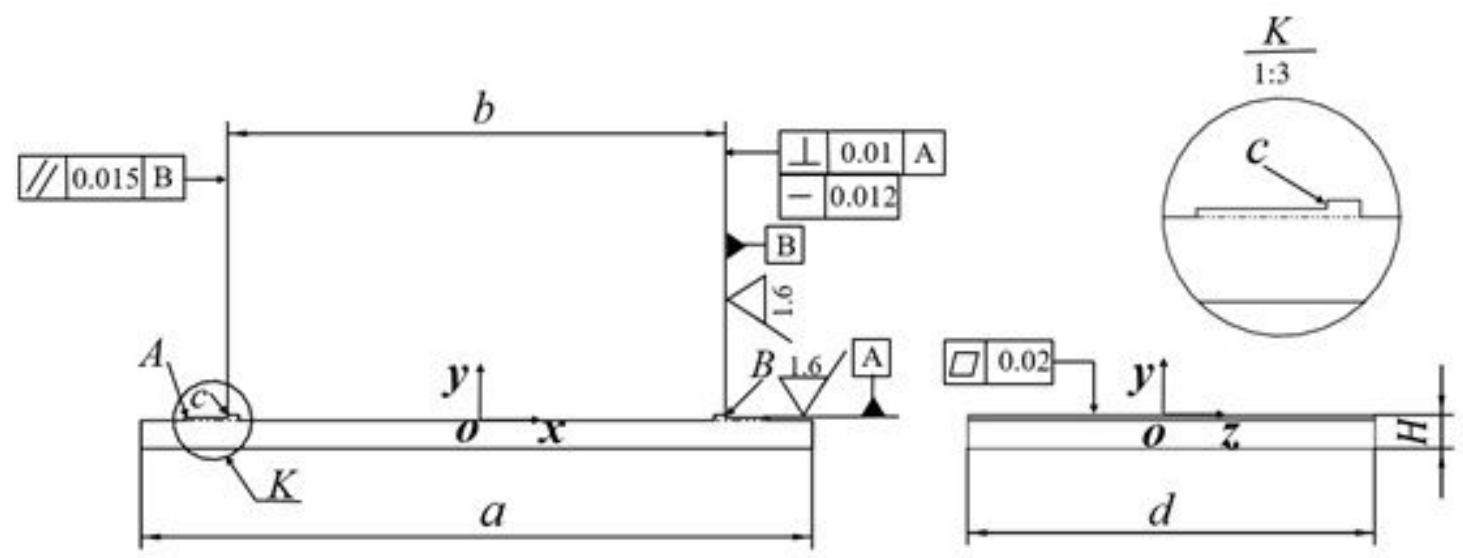

Figure 1

Geometric errors of assembly surface of linear guide rail 


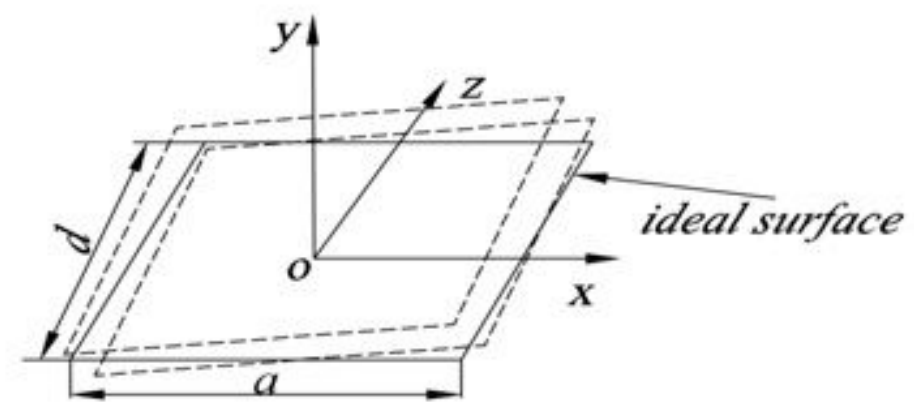

(a)

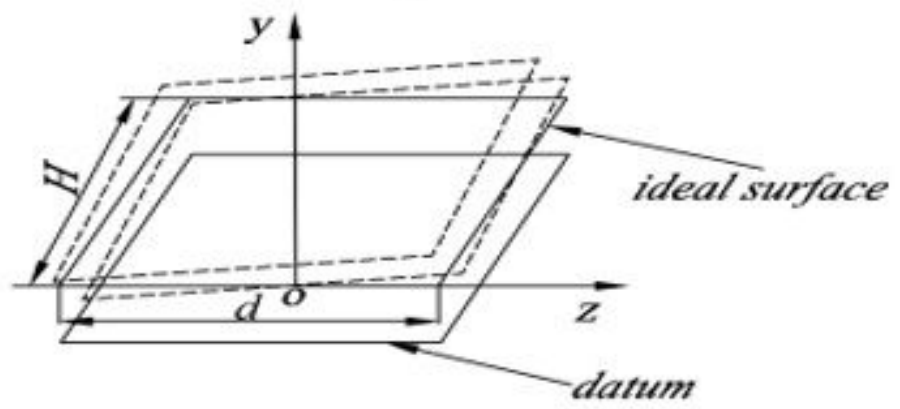

(b)

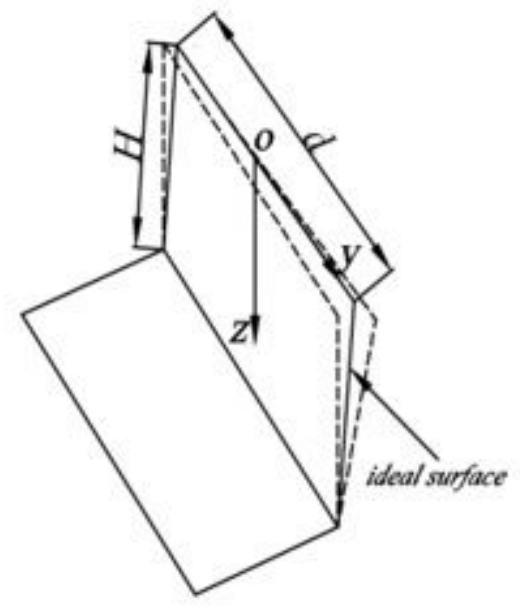

(c)

Figure 2

Variation range (a) flatness; (b) parallelism; (c) perpendicularity 


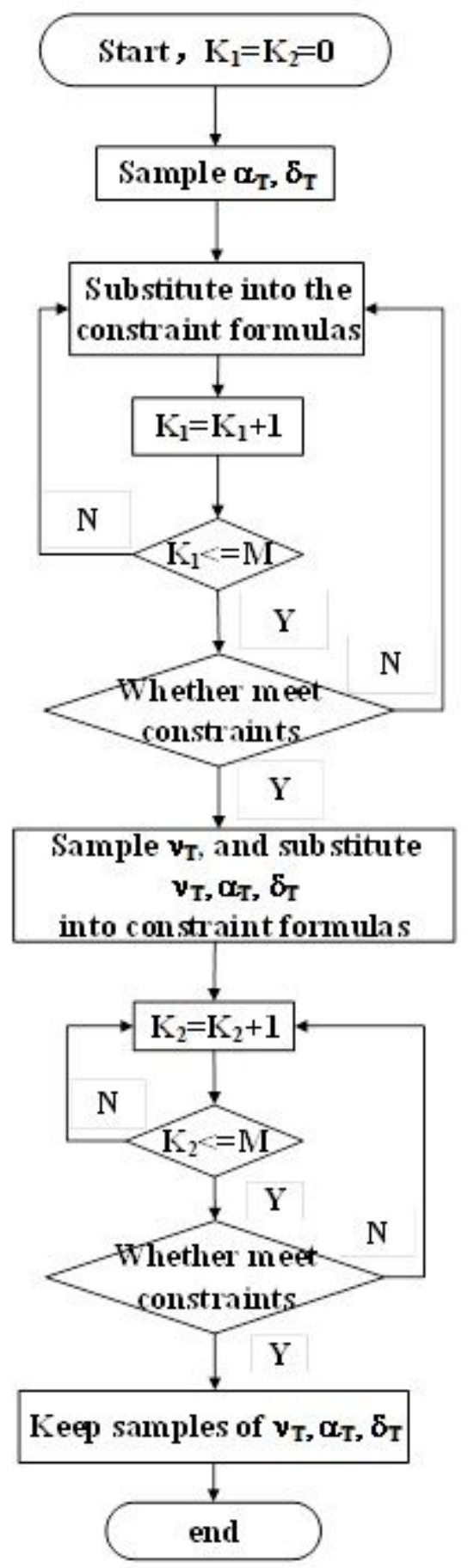

Figure 3

Sampling flow chart of MCS simulation 


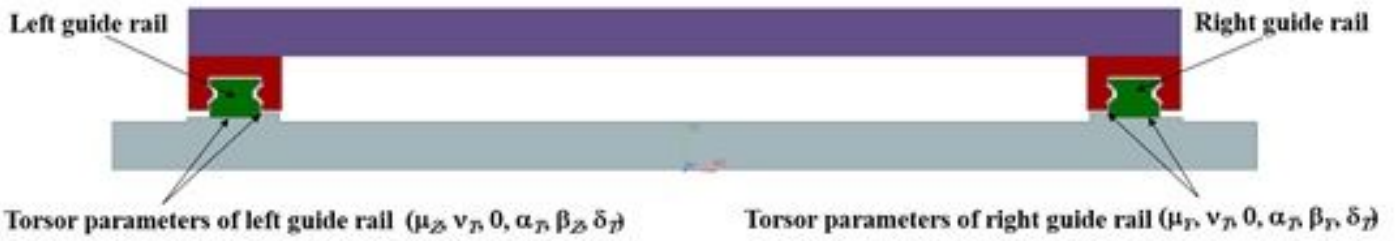

Figure 4

Structure sketch of the linear axis 

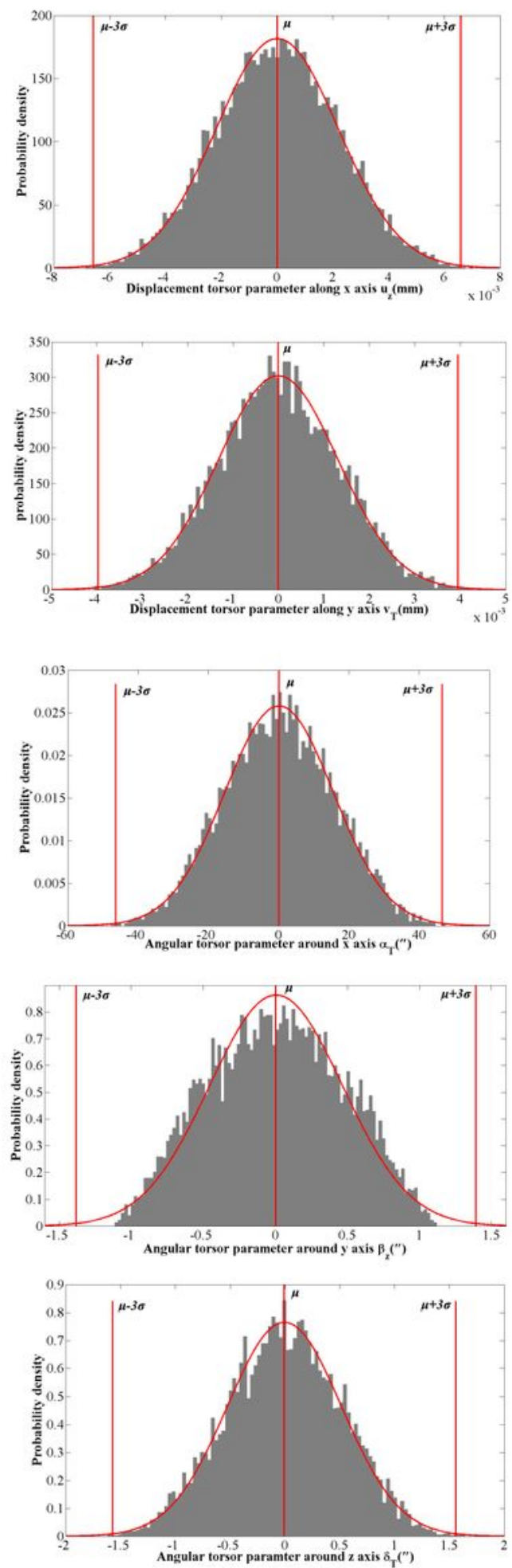

Figure 5

MCS results of comprehensive pose error for left guide rail 

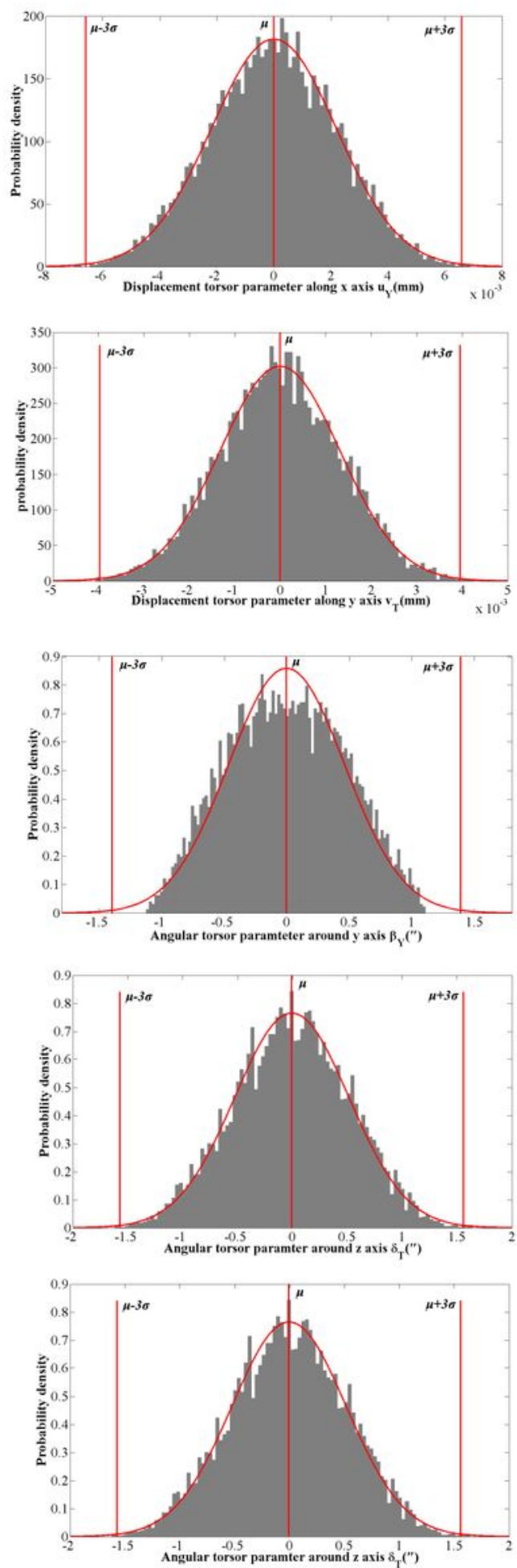

Figure 6

MCS simulation results of comprehensive pose error for left guide rail 


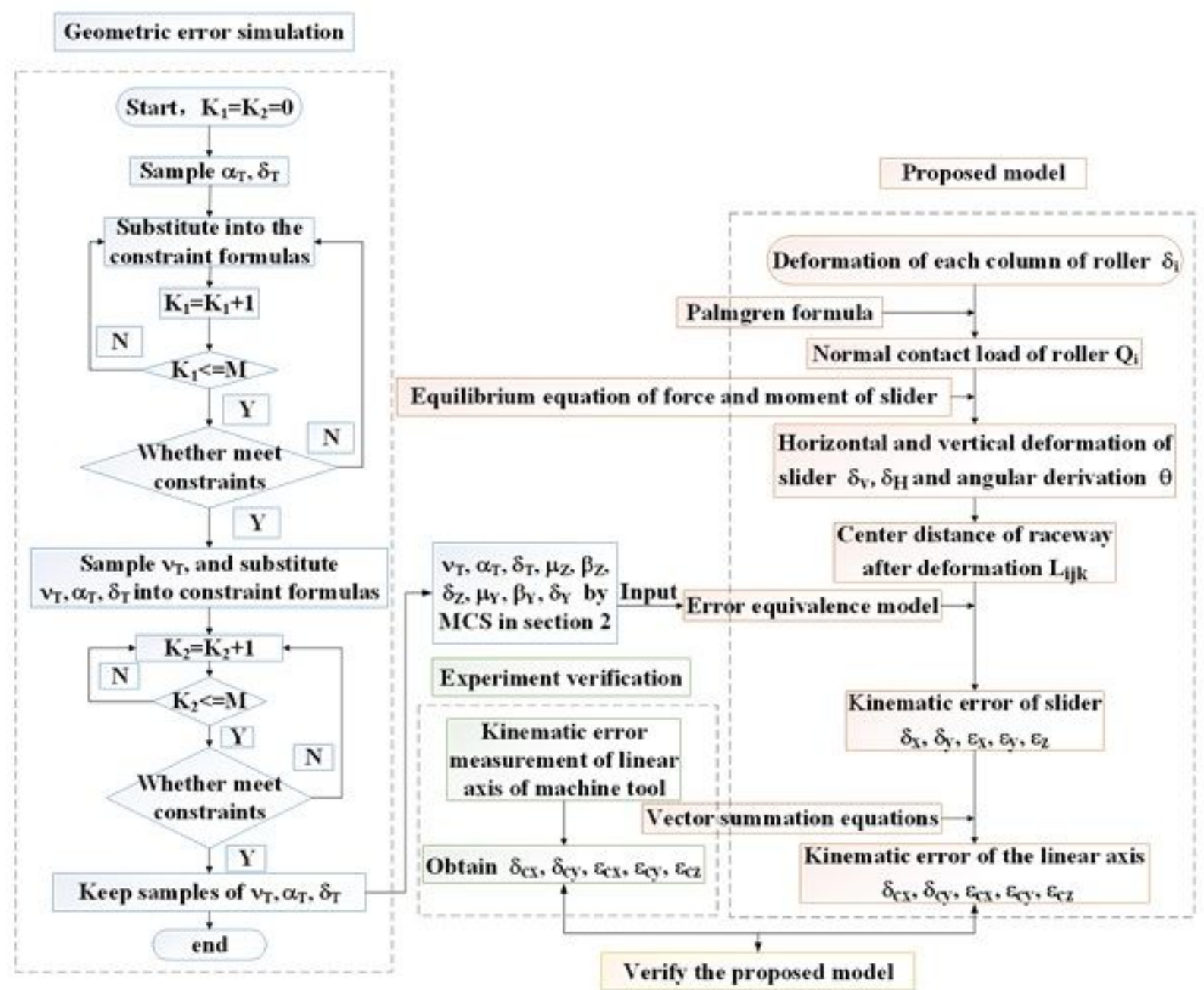

Figure 7

Flow chart of technical route

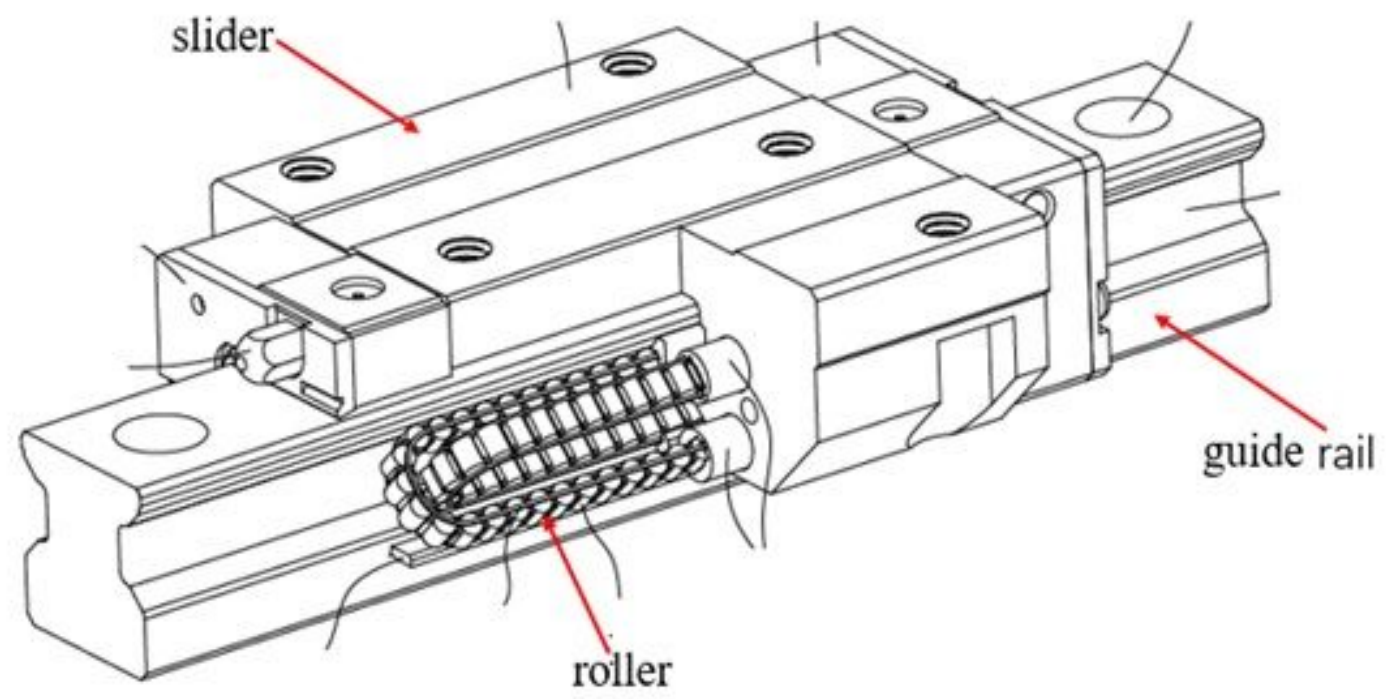


Figure 8

Structure model of linear guide rail

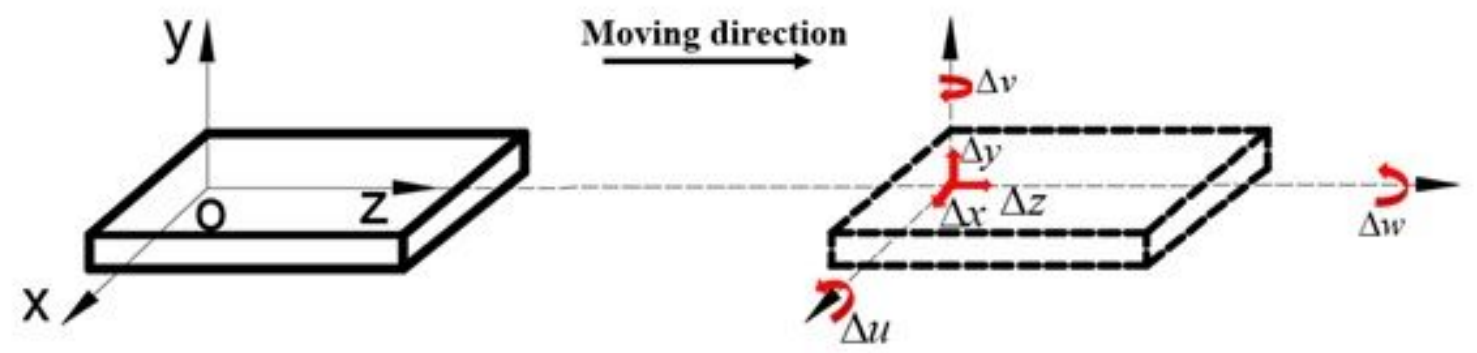

Figure 9

Six directional errors of the linear axis

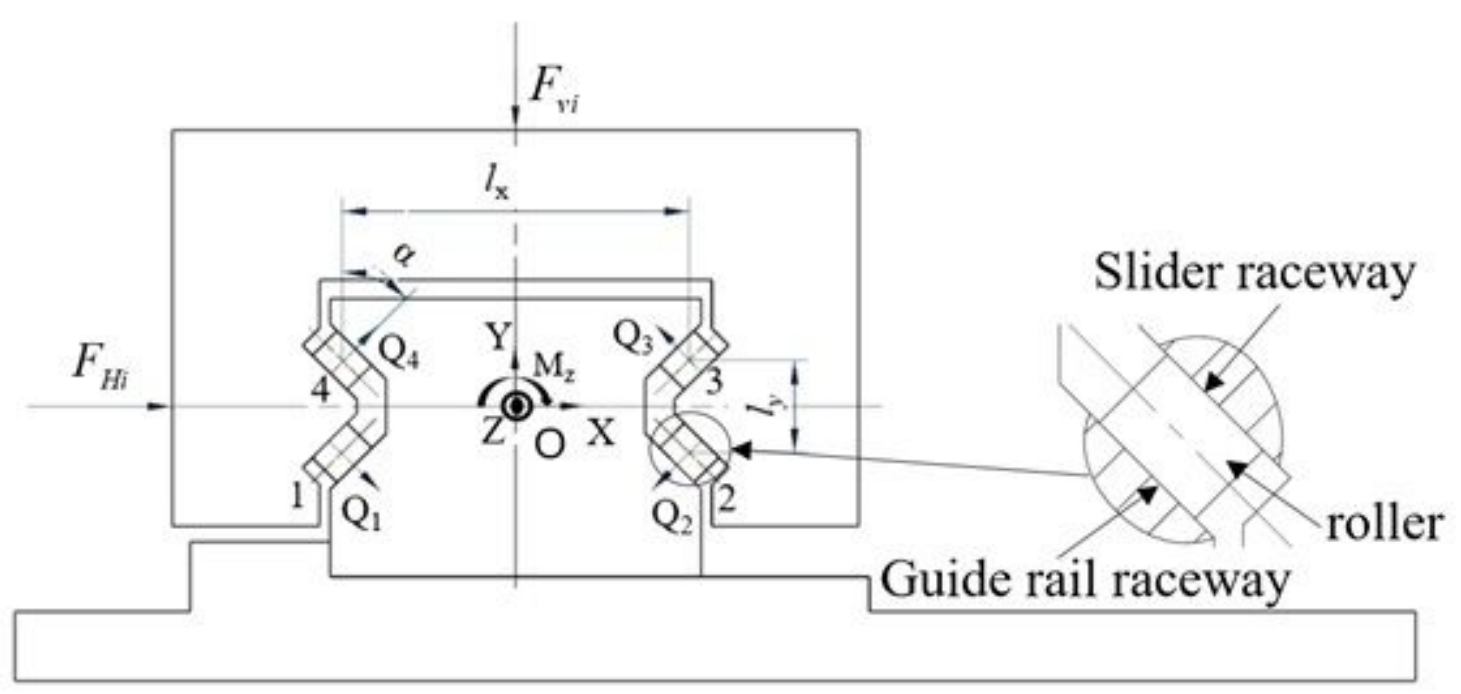

Figure 10

Load analysis of single guide rail 


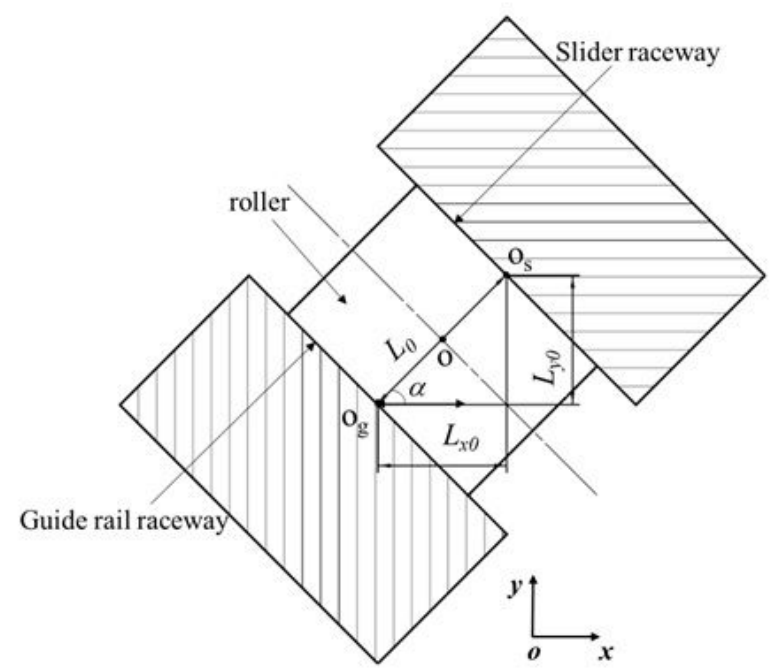

(a)

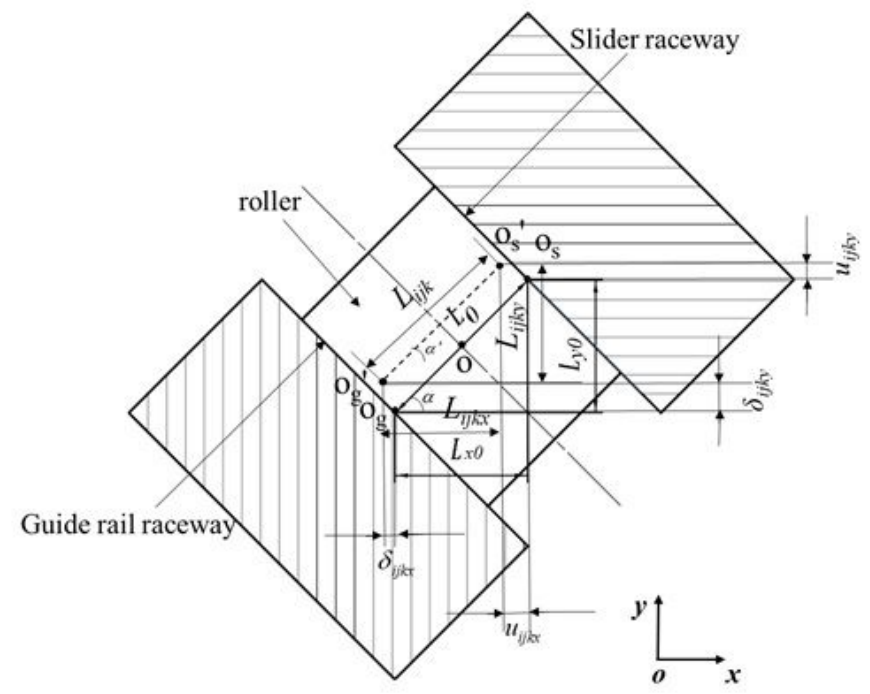

(b)

Figure 11

(a) Contact model of linear guide rail before deformation; (b) Error equivalence model after deformation 


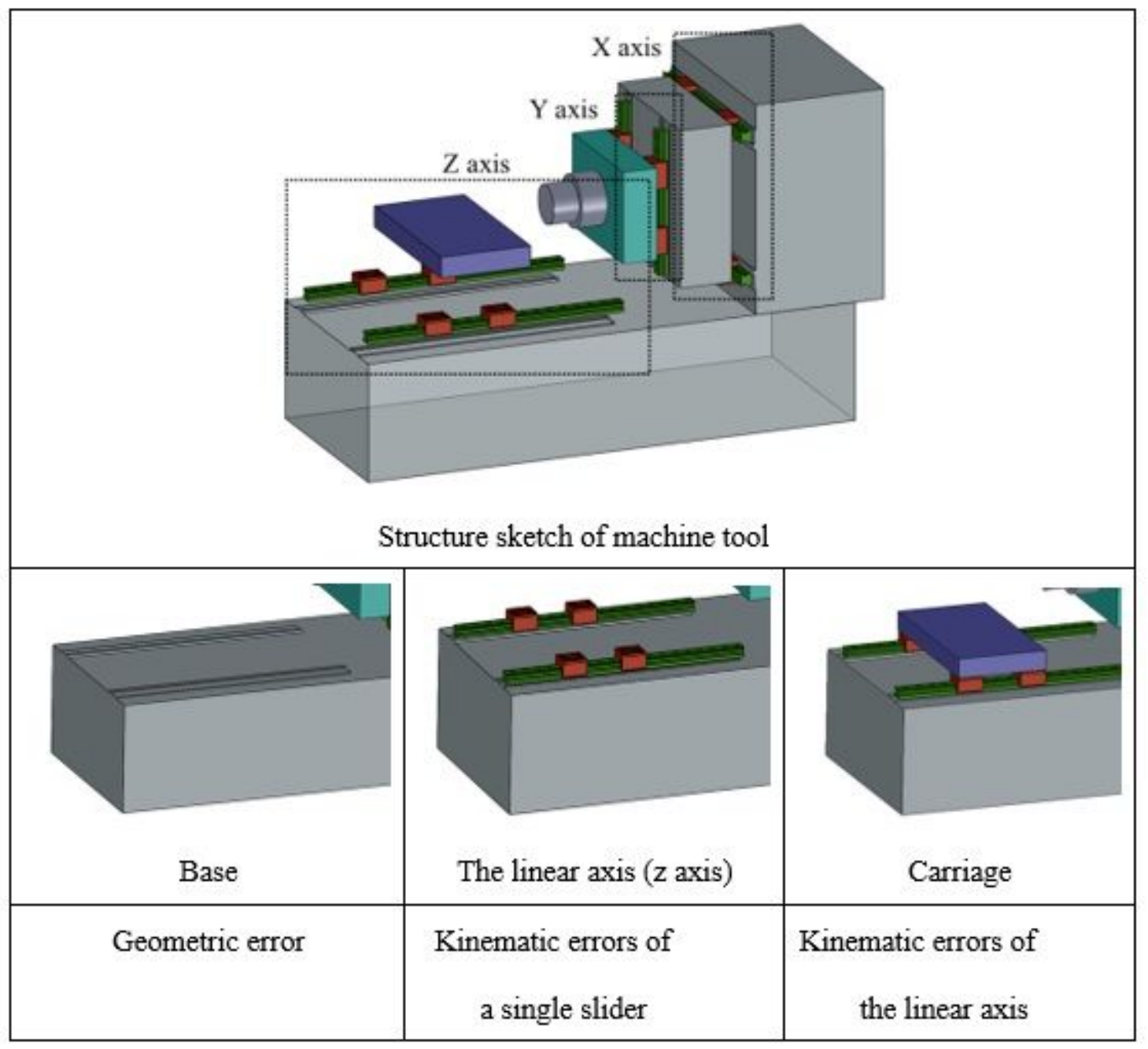

Figure 12

All level errors of the linear axis in machine tool

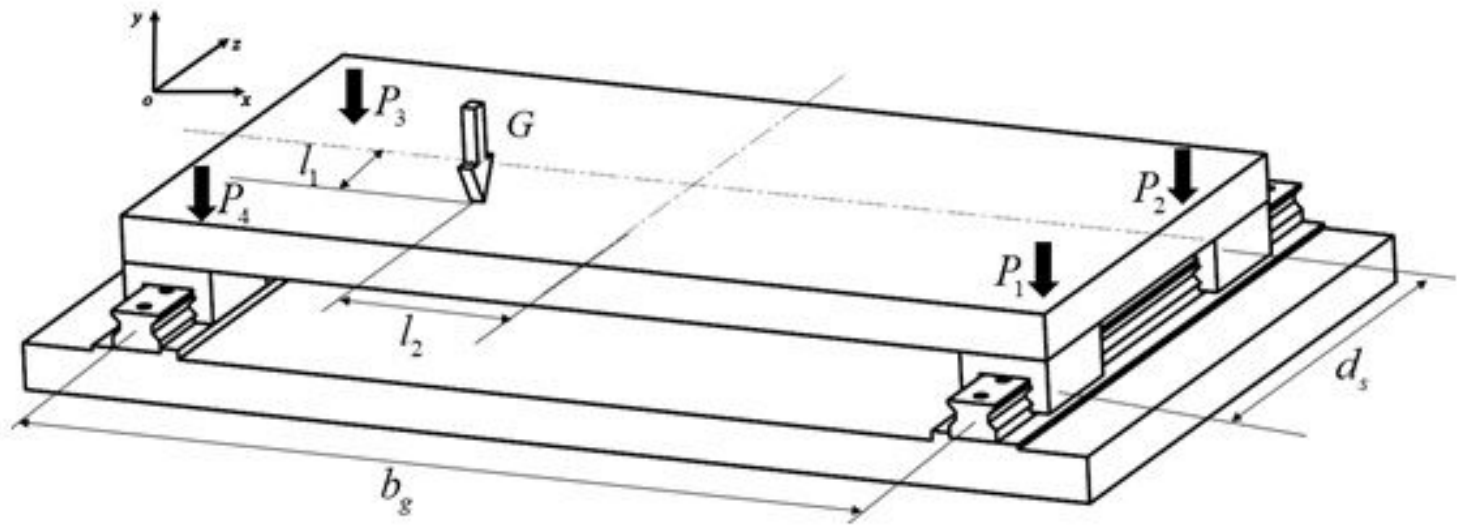

Figure 13

Force analysis of each slider under the workpiece gravity 


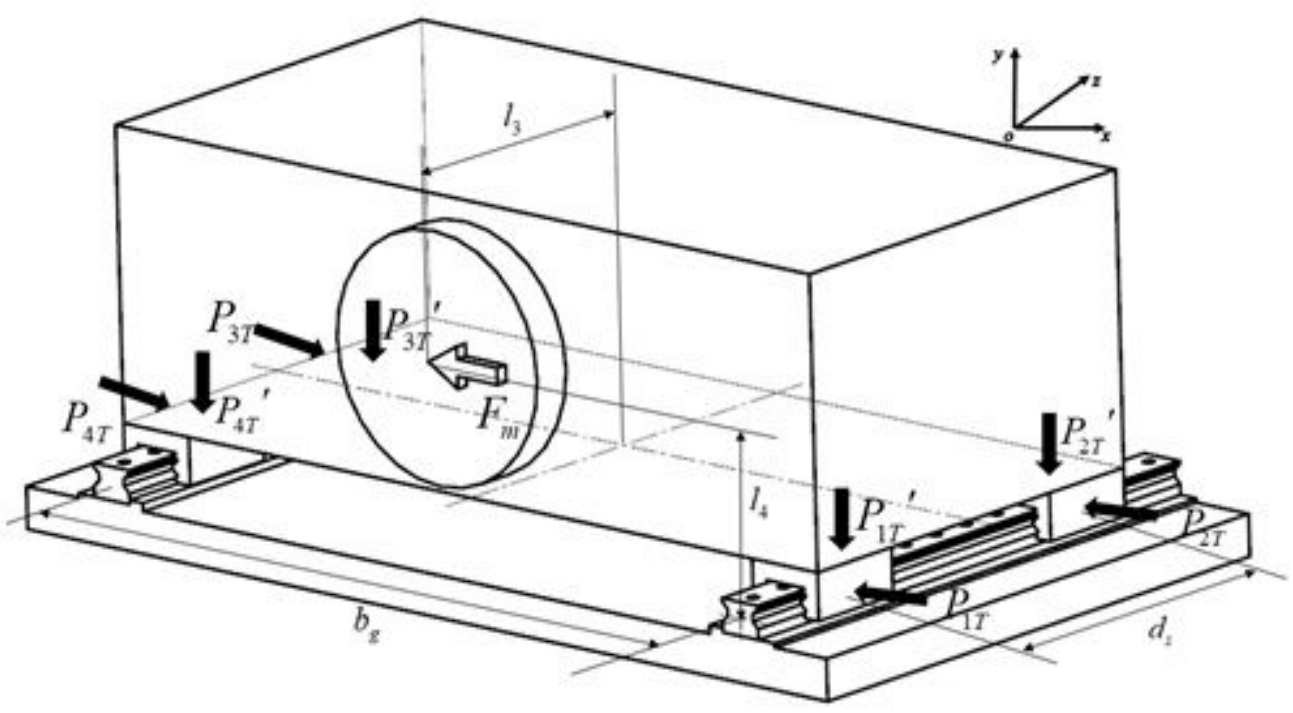

Figure 14

Force analysis of each slider under the milling force

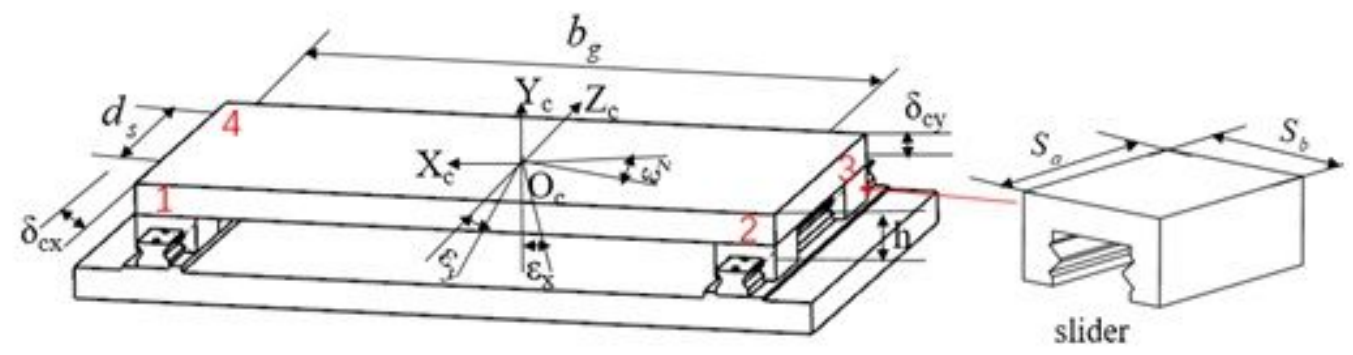

Figure 15

Pose error derivation of carriage 


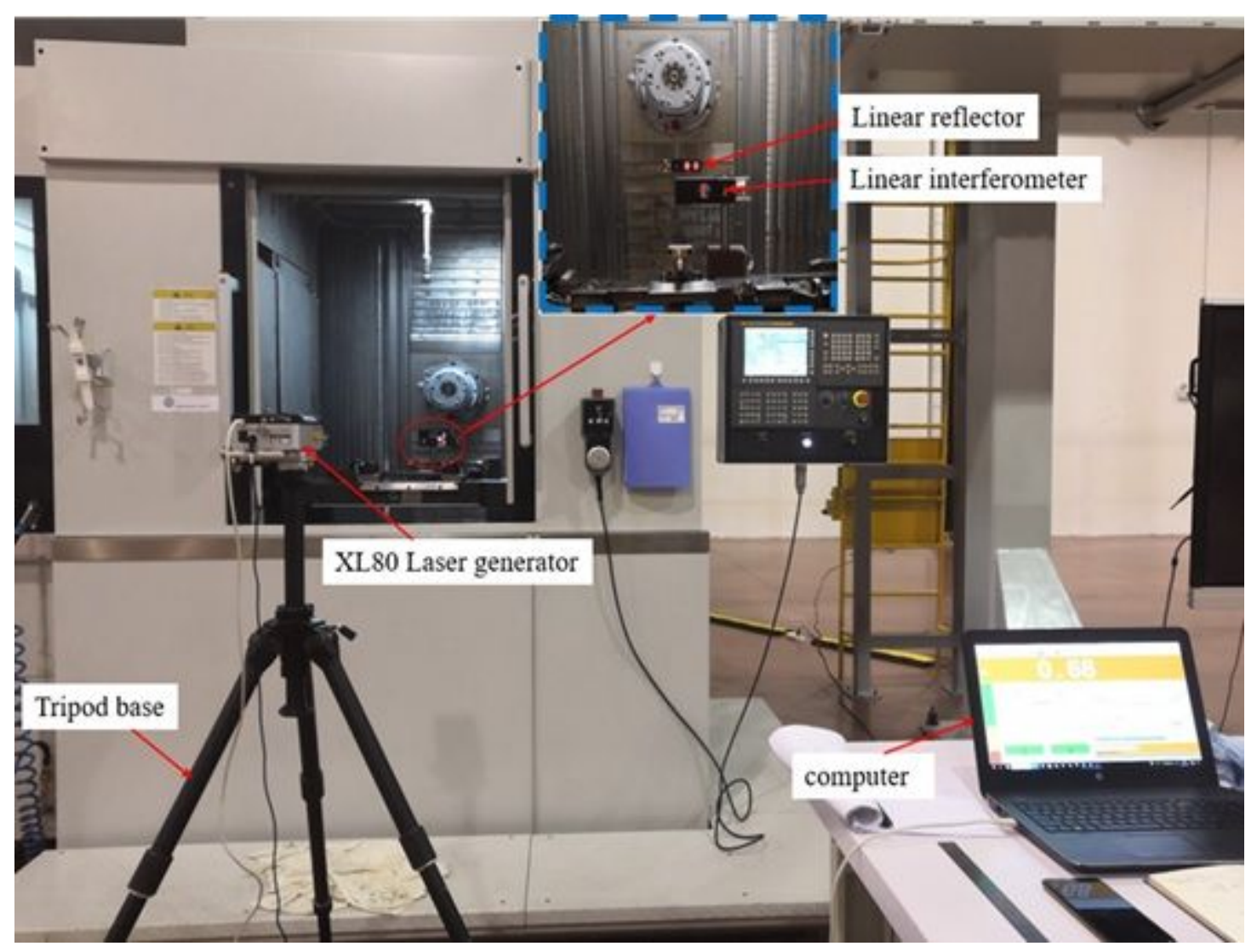

Figure 16

Renishaw equipment of multi-laser interferometer 


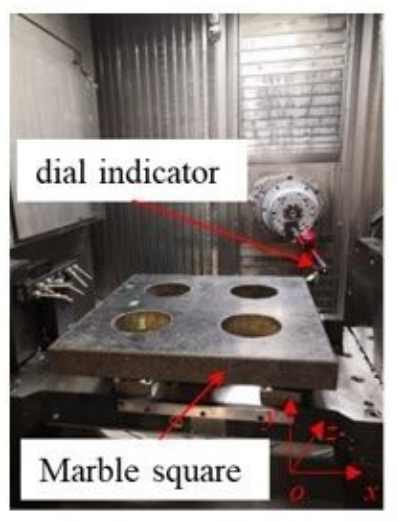

(a)

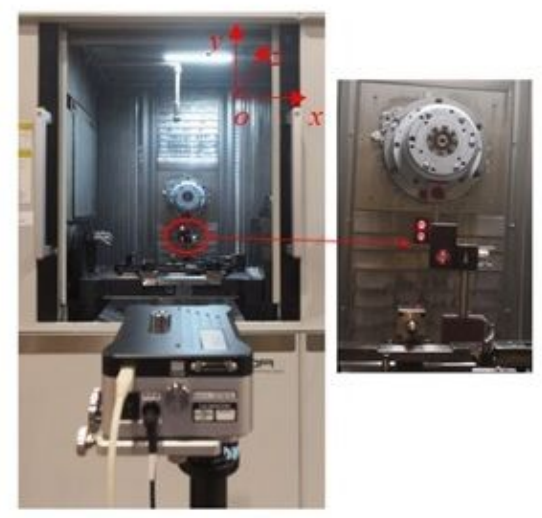

(c)

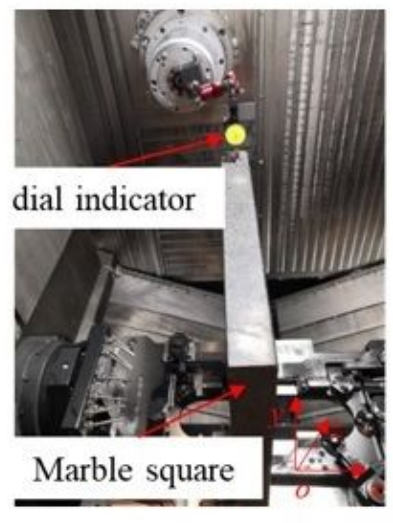

(b)

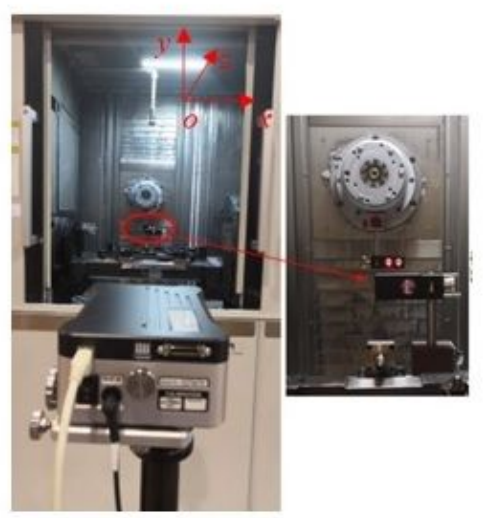

(d)

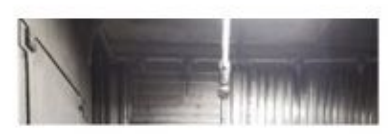

Electronic level meter

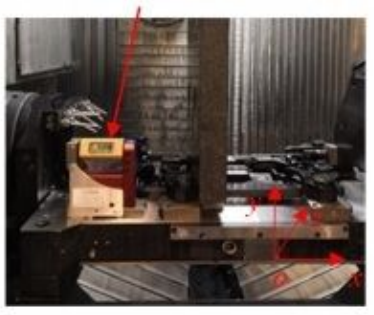

(e)

\section{Figure 17}

Measurement of kinematic error of z-axis of horizontal machine tool (a) Straightness of the yoz plane; (b) Straightness of the xoz plane; (c) yoz plane angular error around x-axis (pitch); (d) xoz plane angular error around $y$-axis (yaw); (e) xoy plane angular error around the $z$ axis (roll) 

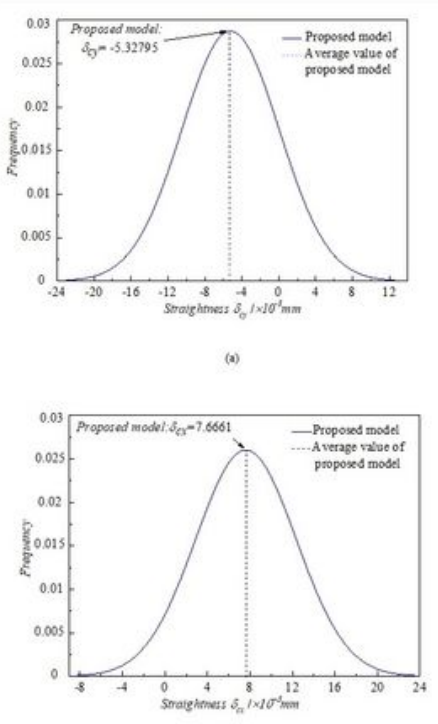

(b)
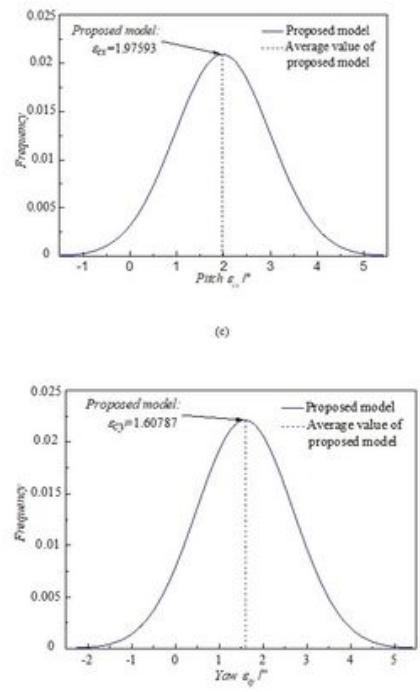

(ब)

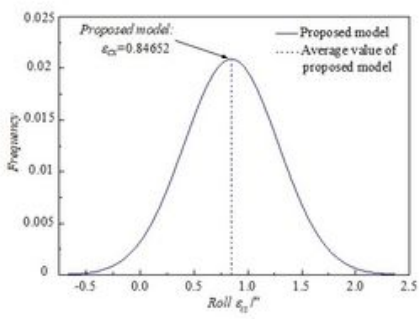

(e)

Figure 18

Calculated kinematic errors of the linear axis: 


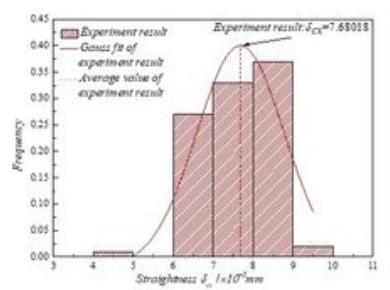

(a)

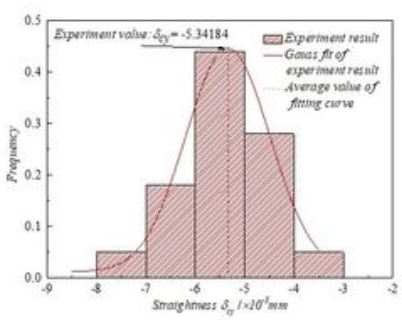

(c)

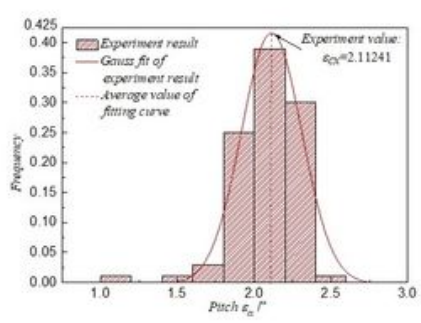

(c)
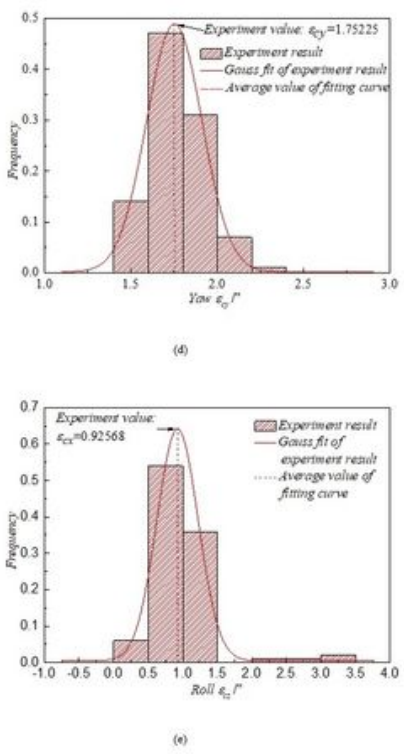

Figure 19

Measured kinematic errors of the linear axis: (a) Straightness (b) Straightness (c) Pitch (d) Yaw (e) Roll 

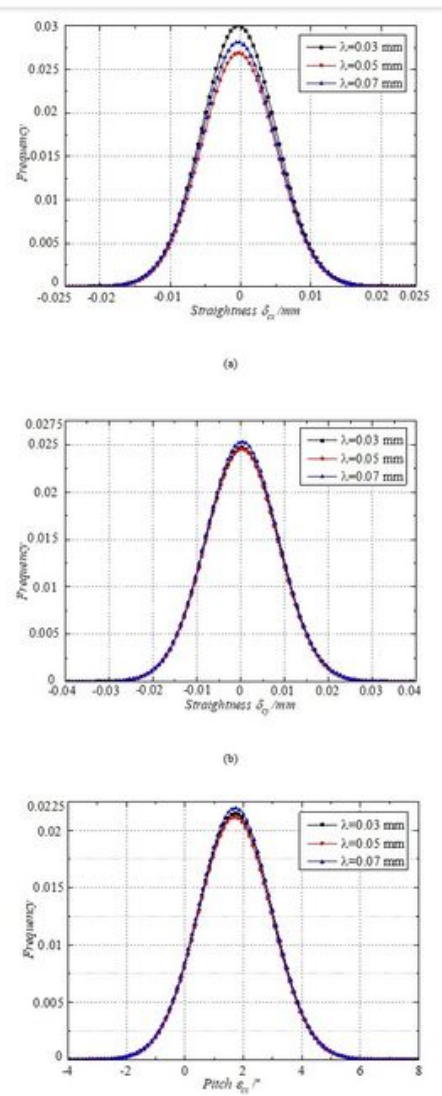

(c)

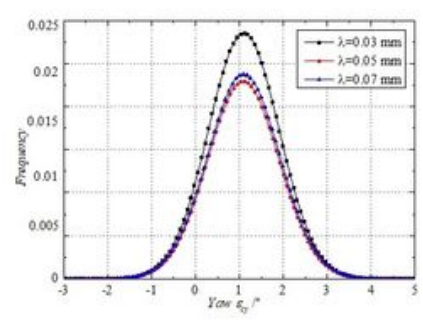

(4)

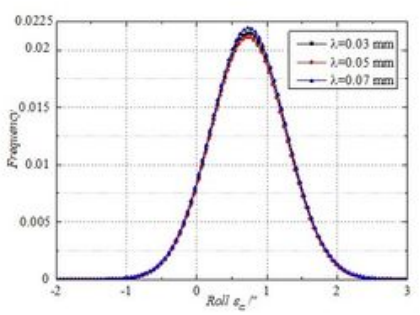

(e)

Figure 20

Kinematic error of the linear axis under different preload (a) Straightness(b) Straightness (c) Pitch (d) Yaw (e) Roll 


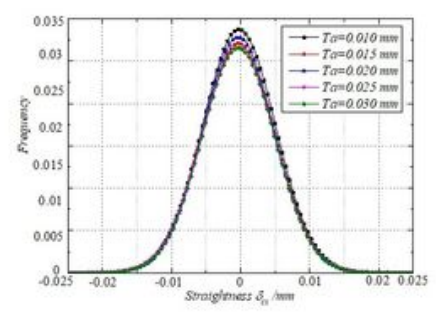

(a)

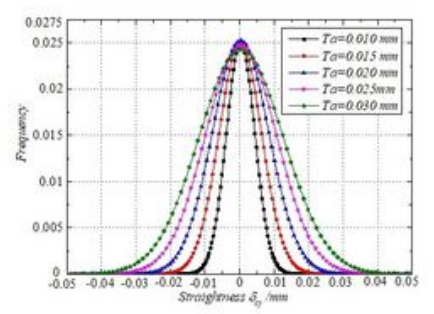

(b)

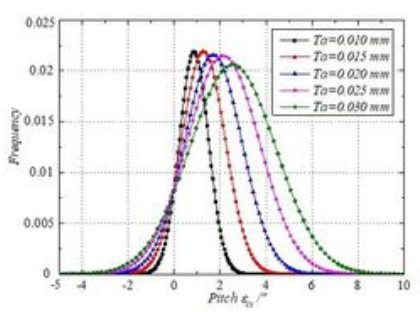

(c)

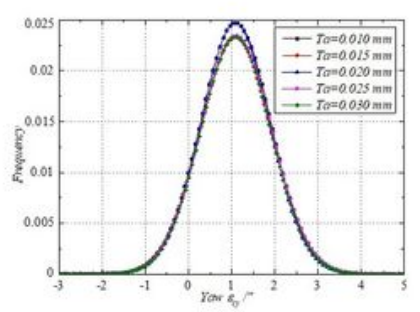

(d)

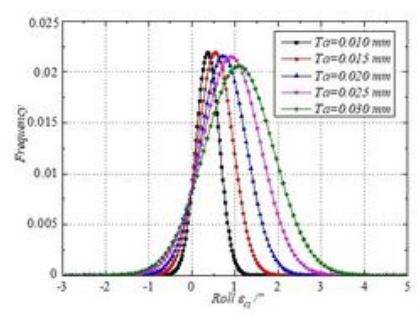

\section{Figure 21}

Kinematic error of the linear axis under different flatness (a) Straightness ; (b) Straightness (c) Pitch (d) Yaw(e) Roll 

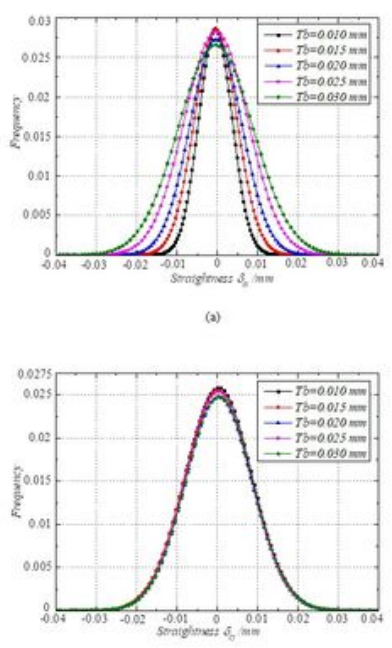

(b)
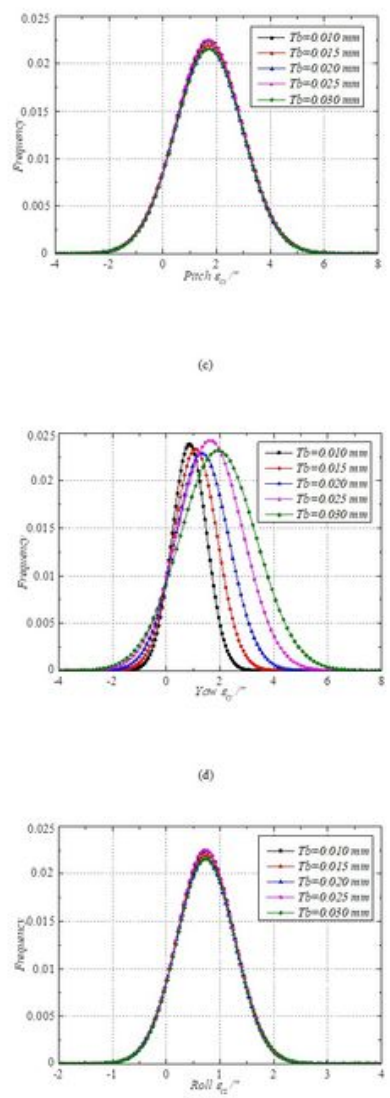

(e)

Figure 22

Kinematic error of the linear axis under different parallelism (a) Straightness (b) Straightness (c) Pitch (d) Yaw (e) Roll 


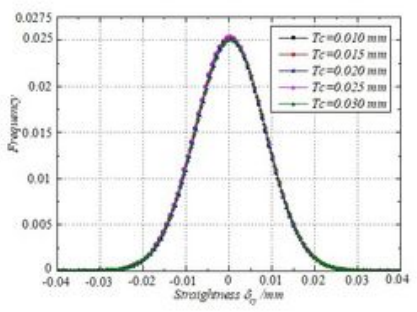

(a)

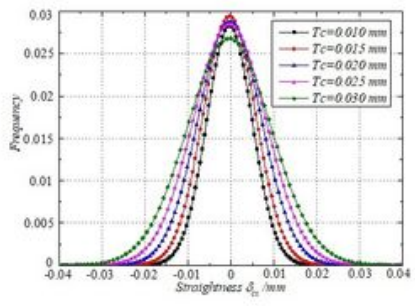

(b)
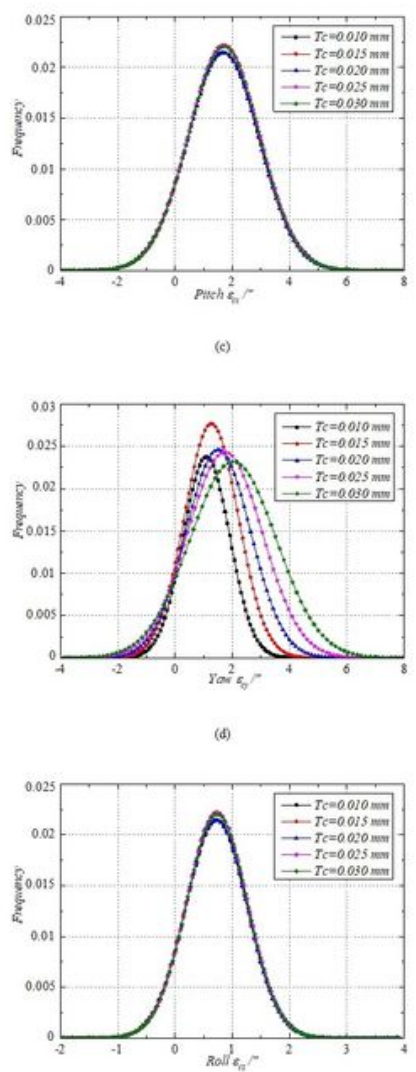

(e)

Figure 23

Kinematic error of the linear axis under different perpendicularity (a) Straightness (b) Straightness (c) Pitch (d) Yaw (e) Roll 

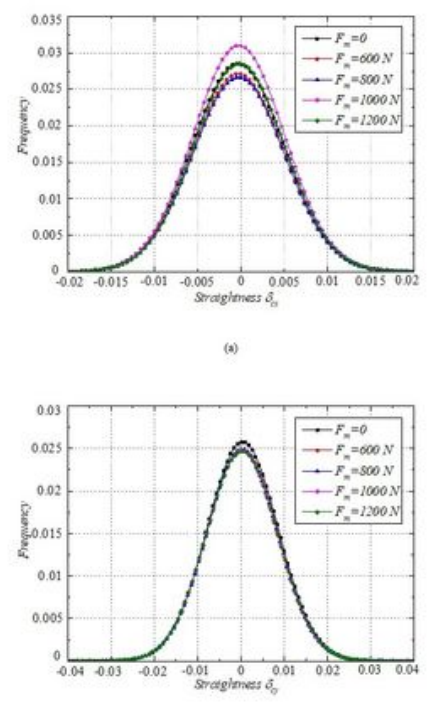

(b)

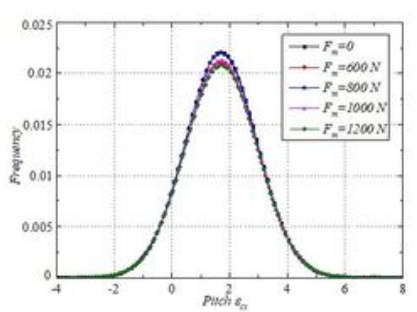

(c)

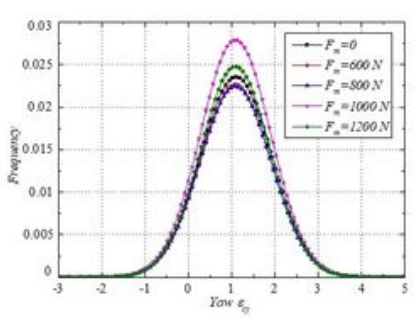

(क)

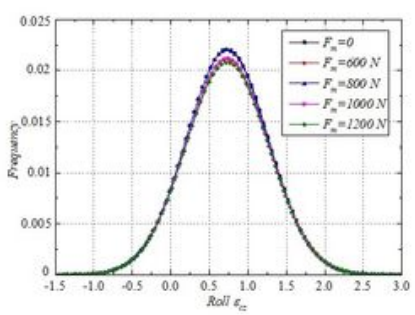

Figure 24

Kinematic error of the linear axis under different milling force (a) Straightness (b) Straightness (c) Pitch (d) Yaw (e) Roll 


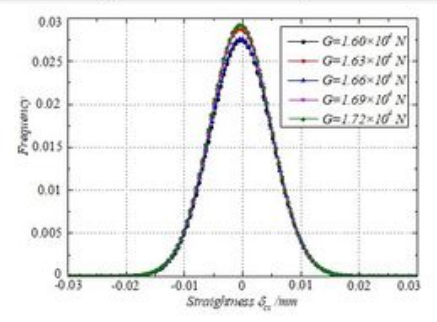

(a)

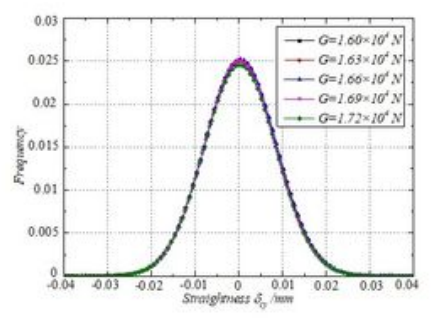

(b)

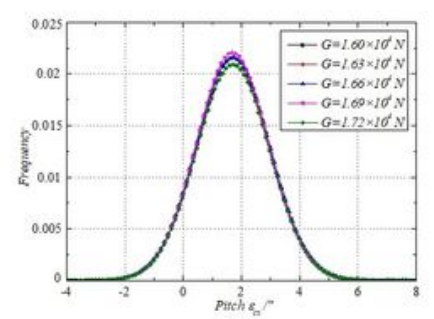

(c)

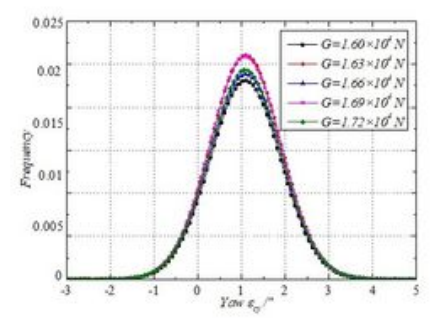

(d)

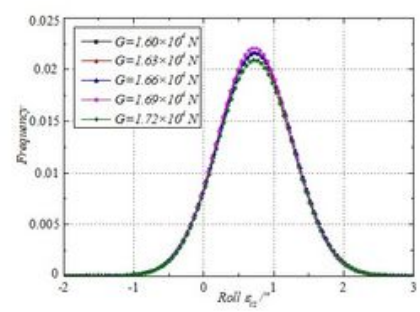

(e)

\section{Figure 25}

Kinematic error of the linear axis under different gravity (a) Straightness (b) Straightness (c) Pitch (d) Yaw (e) Roll 